\title{
Semiparametric instrumental variable estimation of simultaneous equation sample selection models
}

\author{
Lung-Fei Lee \\ Department of Economics, University of Michigan, Ann Arbor, MI 48109-1220. USA
}

(Received June 1991; final version received May 1993)

\begin{abstract}
The identification and estimation of a semiparametric simultaneous equation model with selectivity have been considered. The identification of structural parameters from reduced form parameters in the semiparametric model requires stronger conditions than the usual rank condition in the classical simultaneous equation model or the parametric simultaneous equation sample selection model with normal disturbances. The necessary order condition for identification in the semiparametric model corresponds to the overidentification condition in the classical model. Semiparametric two-stage estimation methods which generalize the two-stage least squares method and the generalized two-stage least squares method for the parametric model are introduced. The semiparametric generalized two-stage least squares estimator is shown to he asymptotically efficient in a class of semiparametric instrumental variable estimators.
\end{abstract}

Key words: Semiparametric model; Sample selection; Simultaneity; Index model; Identification; Instrumental variables; Asymptotic efficiency

JEL classification: $\mathrm{C} 14 ; \mathrm{C} 34$

\section{Introduction}

For the estimation of simultaneous equation sample selection models with parametric (normal) disturbances, several methods are available in the

\footnotetext{
I appreciate having financial support from NSF under grant no. SES-9010516 and SES-9296071 for my research. I would like to thank an anonymous referee for valuable comments and suggestions. The issues in this article are partially motivated by an empirical project under way with my colleagues Professors Mark Rosenzweig and Mark M. Pitt.
} 
econometric literature, e.g., Lee, Maddala, and Trost (1980), Lee (1981), Amemiya (1983), Newey (1987), and Blundell and Smith (1989). The approach introduced in Lee, Maddala, and Trost (1980) combines Heckman's two-stage and Theil's two-stage least squares procedures. Amemiya (1983) considered a class of estimators derived from modified minimum distance procedures. Relative efficiency of such procedures has been considered in Lee (1981), Amemiya (1983), Newey (1987), and Blundell and Smith (1989).

In this article, we will consider instrumental variable (IV) methods for the estimation of simultaneous equation sample selection models without parametric distributional assumptions. Semiparametric instrumental variable methods for the estimation of sample selection models have been considered in Powell (1987) (see also Robinson, 1988). In Powell (1987), since his interest is on general semiparametric instrumental variable methods, he has not focused attention on any specific simultaneous equation structures of the model. Many interesting issues, such as the rank identification condition, which are wellknown for the classical simultaneous equation model, have not been addressed for the semiparametric simultaneous equation model. In this article, we are interested in the specific structure of simultaneous equation sample selection models. We investigate the problem of structural parameter identification, the role of identification conditions on semiparametric instrumental variable estimation, and the proper construction of instrumental variables from the system. We will also investigate the possible generalization of the (semiparametric) two-stage least squares estimation method and the construction of efficient semiparametric instrumental variable estimators.

\section{Semiparametric simultaneous equation models with selectivity and instrumental variable estimation}

In this article, our discussion will focus on the estimation of a single equation. The estimation of multiple equations can be easily generalized. Consider a single structural equation:

$$
y^{*}=z^{*} \alpha_{0}+x J \gamma_{0}+u_{1},
$$

where $y^{*}$ is a latent endogenous variable, $z^{*}$ is a $G_{1}$-dimensional row vector of latent endogenous variables not including $y^{*}, x$ is a $K$-dimensional row vector consisting of all exogenous variables in the system, and $x J$, where $J$ is a selection matrix, represents the subset of exogenous variables included in this structural equation. The reduced form equation of $z^{*}$ is

$$
z^{*}=x \Pi_{2}+v_{2}
$$

where $\Pi_{2}$ is a $K \times G_{1}$ matrix and $v_{2}$ is a $G_{1}$ row vector of disturbances. The endogenous variables $y^{*}$ and $z^{*}$ are well-defined in the whole population, but 
their sample observations $y$ and $z$ are subject to selection. The latent selection equation is

$$
d^{*}=x \zeta_{0}+\varepsilon,
$$

where $d^{*}$ is a latent variable. The values of $y$ and $z$ are observable if and only if $d^{*}>0$. As in Ichimura (1987) and Powell (1987), we consider the index model framework where the joint distribution of $\left(u_{1}, v_{2}, \varepsilon\right)$ conditional on $x$ can be a function of the index $x \zeta_{0}$. Such a framework is slightly more general than the case where the disturbances are independent of $x$.

Conditional on $d_{i}^{*}>0$ and $x_{i},(2.1)$ implies that

$$
y_{i}=z_{i} \alpha_{0}+x_{i} J \gamma_{0}+\mathrm{E}\left(u_{1 i} \mid x_{i} \zeta_{0}, d_{i}^{*}>0\right)+u_{d i}
$$

where

$$
u_{d i}=u_{1 i}-\mathrm{E}\left(u_{1 i} \mid x_{i} \zeta_{0}, d_{i}^{*}>0\right) .
$$

Let $K(\cdot)$ be a kernel function with a bandwidth parameter $a_{n}$ (Silverman, 1986, or Bierens, 1985). Let $w=(z, x J)$ and $\beta_{0}=\left(\alpha_{0}, \gamma_{0}\right)$. For any possible value $(\beta, \zeta)$ of $\left(\beta_{0}, \zeta_{0}\right)$, the conditional expectation function $\mathrm{E}\left(y-w \beta \mid x \zeta=x_{i} \zeta\right.$, $d^{*}>0$ ) of $y-w \beta$, conditional on the random variables $x_{\zeta}^{\zeta}$ and $d^{*}>0$ evaluated at the point $x_{i} \zeta$, can be estimated by the following nonparametric regression function:

$$
\mathrm{E}_{n}\left(y-w \beta \mid x_{i} \zeta\right)=\frac{\sum_{j \neq i}^{n}\left(y_{j}-w_{j} \beta\right) K\left(\frac{x_{i} \zeta-x_{j} \zeta}{a_{n}}\right)}{\sum_{j \neq i}^{n} K\left(\frac{x_{i} \zeta-x_{j} \zeta}{a_{n}}\right)},
$$

where $n$ is the sample size for the observations of $(y, z, x)$ conditional on $d^{*}>0$ (Nadaraja, 1964; Watson, 1964). Given a $\sqrt{n}$-consistent estimate $\hat{\zeta}$ of $\zeta$, Powell (1987) has proposed an instrumental variable method for the estimation of $\beta_{0}$ from the following equation:

$$
y_{i}-\mathrm{E}_{n}\left(y \mid x_{i} \hat{\zeta}\right)=\left(w_{i}-\mathrm{E}_{n}\left(w \mid x_{i} \hat{\zeta}\right)\right) \beta_{0}+\hat{u}_{n i}
$$

where

$$
\mathrm{E}_{n}\left(s \mid x_{i} \zeta\right)=\sum_{j \neq i}^{n} s_{j} K\left(\frac{x_{i} \zeta-x_{j} \zeta}{a_{n}}\right) / \sum_{j \neq i}^{n} K\left(\frac{x_{i} \zeta-x_{j} \zeta}{a_{n}}\right)
$$

for any random variable $s$ (see also Robinson, 1988). Instrumental variable methods require instrumental variables for $w_{i}-\mathrm{E}_{n}\left(w \mid x_{i} \hat{\zeta}\right)$ (Powell, 1987). A simple instrumental variable estimator with instrumental variables $p$ can be

$$
\left(\sum_{i=1}^{n} p_{i}^{\prime}\left(w_{i}-\mathrm{E}_{n}\left(w \mid x_{i} \hat{\zeta}\right)\right)\right)^{-1} \sum_{i=1}^{n} p_{i}^{\prime}\left(y_{i}-\mathrm{E}_{n}\left(y \mid x_{i} \hat{\zeta}\right)\right) \text {. }
$$


However, due to the technical difficulty of handling the denominator in the nonparametric regression function in (2.6), some modifications are needed to overcome the difficulty. Various ways have been introduced in the literature. Powell (1987) uses the denominator in (2.6) as the weight in the summations of (2.8) so as to cancel the denominator of (2.6). An alternative suggestion is to trim the tails of the distribution of $x$ or the index $x \hat{\zeta}$ (Robinson, 1988; Klein and Spady, 1987; Ichimura and Lee, 1991). In this article, trimming will be applied to the index $x \hat{\zeta}$ when its values are greater than some upper quantile or less than some lower quantile (see Sections A.2 and A.3 of the Appendix). Suppose that $t_{n}\left(x_{i} \hat{\zeta}, \hat{\xi}_{n}\right)$ is a trimming indicator with value 0 when $x_{i} \hat{\zeta}$ is deleted, where $\hat{\xi}_{n}$ is a vector of sample quantiles. A simple unweighted instrumental variable estimator is

$$
\begin{aligned}
\hat{\beta}_{p}= & \left(\sum_{i=1}^{n} t_{n}\left(x_{i} \hat{\zeta}, \hat{\xi}\right) p_{i}^{\prime}\left(w_{i}-\mathrm{E}_{n}\left(w \mid x_{i} \hat{\zeta}\right)\right)\right)^{-1} \\
& \times \sum_{i=1}^{n} t_{n}\left(x_{i} \hat{\zeta}, \hat{\xi}_{n}\right) p_{i}^{\prime}\left(y_{i}-\mathrm{E}_{n}\left(y \mid x_{i} \hat{\zeta}\right)\right) .
\end{aligned}
$$

The trimming procedure is preferred to the Powell's procedure. The weighting scheme in Powell (1987) has nothing to do with the variance of the disturbance $\hat{u}_{n i}$ in (2.7). With the trimming procedure, weighting estimation method which incorporates heteroskedastic variances can be introduced in subsequent sections.

As in the classical simultaneous equation model, the consistency of IV estimators depends on proper instruments constructed from the list of exogenous variables $x$ in the system. Consistency of the IV estimators is possible only if the structural equation is identifiable. In subsequent sections, we will first address the identification problem of this system. Problems on how to select proper instrumental variables and the construction of efficient IV estimation of (2.7) will then be considered.

The estimation method can be generalized to cover more general cases where the selection mechanism is determined by several inequality conditions, for example, models with polychotomous or sequential choices. For the general case, $d^{*}$ in (2.3) is a finite-dimensional (row) vector of latent equations. The samples of $y$ and $z$ are observed if and only if $d^{*}>0$. The implied regression equation (2.4) becomes a model with multiple indices (Stoker, 1986; Ichimura and Lee, 1991). $x \zeta$ will represent a vector of indices with $\zeta$ being a matrix. The semiparametric estimation method above can be generalized. $K(\cdot)$ will now be a higher-dimensional kernel with the dimension of $x \zeta$, and the trimming will be applied to all the indices in $x \zeta$. The bandwidth $a_{n}$ needs to be wider in the nonparametric regression estimation in (2.6). The detailed analysis in the Appendix is applicable to the general model. 


\section{Identification}

Let

$$
y^{*}=x \pi_{1}+v_{1}
$$

be the reduced form equation for $y^{*}$. As in the classical simultaneous equation model, the identification of structural parameters is directly related to the reduced form parameters. Within the index model framework, identification of (2.2), (2.3), and (3.1) has been considered in Ichimura (1987), Chamberlain (1986), Powell (1987), and Ichimura and Lee (1991). Conditional on $d^{*}>0$ and $x$,

$$
\mathrm{E}\left(y \mid x, d^{*}>0\right)=x \pi_{1}+\mathrm{E}\left(v_{1} \mid x \zeta_{0}, x \zeta_{0}>-\varepsilon\right)
$$

and

$$
\mathrm{E}\left(z \mid x, d^{*}>0\right)=x \Pi_{2}+\mathrm{E}\left(v_{2} \mid x \zeta_{0}, x \zeta_{0}>-\varepsilon\right) .
$$

As shown in Ichimura (1987) for the single-index model, $\zeta_{0}$ in the selection equation (2.3) can best be identified up to an unknown scale. When the re gressors in $x$ are all qualitative variables, $\zeta_{0}$ cannot even be identificd up to a scale. Therefore, we consider only the model where a relevant continuous exogenous variable is present in the index $x \zeta_{0}$. As the coefficients in the index can only be identified up to a scale, normalization is needed. A convenient normalization (Ichimura, 1987) is to set the coefficient of a continuous exogenous variable to the unity. Contrary to the classical simultaneous equation model, the reduced form parameter vectors $\pi_{1}$ and $\Pi_{2}$ in (3.1) and (2.2) are not identifiable. This is so because $x \pi_{1}$ and $x \zeta_{0}$ contain the same set of variables $x$ and they cannot be distinguished from each other in (3.2). Similarly, this is so for $x \Pi_{2}$ and $x \zeta_{0}$. This identification problem has been studied in Ichimura and Lee (1991) and Powell (1987) in the analysis of index models with nonparametric regression functions. The same conclusion has been derived in Chamberlain (1986) from the nonparametric likelihood function of the model.

Even though $\pi_{1}$ and $\Pi_{2}$ are not identifiable, some transformations of them can be identified. With the normalization suggested by Ichimura (1987), let

$$
x \zeta_{0}=x_{(1)}+x_{(2)} \delta_{0}
$$

where $x_{(1)}$ is a relevant continuous exogenous variable in $x=\left(x_{(1)}, x_{(2)}\right)$ and $x_{(2)}$ is the remaining subvector of $x$. Conformably, $x \pi_{1}=x_{(1)} \pi_{11}+x_{(2)} \pi_{12}$, $x \Pi_{2}=x_{(1)} \pi_{21}^{\prime}+x_{(2)} \Pi_{22}$, and $x \gamma_{0}=x_{(1)} \gamma_{0,1}+x_{(2)} \gamma_{0,2}$. The reduced form equation $(3.1)$ can be rewritten into

$$
y^{*}=x_{(2)} \pi_{1}^{*}+v_{1}^{*}
$$

where $\pi_{1}^{*}=\pi_{12}-\delta_{0} \pi_{11}$ and $v_{1}^{*}=v_{1}+x \zeta_{0} \pi_{11}$. Similarly,

$$
z^{*}=x_{(2)} \Pi_{2}^{*}+v_{2}^{*},
$$


where $\Pi_{2}^{*}=\Pi_{22}-\delta_{0} \pi_{21}^{\prime}$ and $v_{2}^{*}=v_{2}+x \zeta_{0} \pi_{21}^{\prime}$. It follows that

and

$$
\mathrm{E}\left(y \mid x, d^{*}>0\right)=x_{(2)} \pi_{1}^{*}+\mathrm{E}\left(v_{1}^{*} \mid x \zeta_{0}, d^{*}>0\right)
$$

$$
\mathrm{E}\left(z \mid x, d^{*}>0\right)=x_{(2)} \Pi_{2}^{*}+\mathrm{E}\left(v_{2}^{*} \mid x_{\zeta_{0}}, d^{*}>0\right) .
$$

The index $x \zeta_{0}$ is distinguishable from $x_{(2)} \pi_{1}^{*}$ and $x_{(2)} I_{2}^{*}$, because $x_{(1)}$ appears only in $x \zeta_{0}$ but neither in $x_{(2)} \pi_{1}^{*}$ nor in $x_{(2)} \Pi_{2}^{*}$. The transformed parameters $\pi_{1}^{*}$ and $\Pi_{2}^{*}$ and $\delta_{0}$ are identifiable.

The structural parameters $\alpha$ and $\gamma$ are related to the reduced form parameters $\pi_{1}^{*}, \Pi_{2}^{*}$, and $\delta$. Substituting (3.5) into the structural equation $y^{*}$ (before imposing any explicit exclusion restrictions), we have

$$
\begin{aligned}
y^{*} & =z^{*} \alpha_{0}+x \bar{\gamma}_{0}+u_{1} \\
& =\left(x_{(2)} \Pi_{2}^{*}+v_{2}^{*}\right) \alpha_{0}+x_{(1)} \gamma_{0,1}+x_{(2)} \gamma_{0,2}+u_{1} \\
& =x_{(2)}\left(\Pi_{2}^{*} \alpha_{0}-\gamma_{0,1} \delta_{0}+\gamma_{0,2}\right)+u_{1}^{*},
\end{aligned}
$$

where $u_{1}^{*}=u_{1}+v_{2}^{*} \alpha_{0}+\gamma_{0,1} x \zeta_{0}$ and $\bar{\gamma}_{0}=\left(\gamma_{0,1}^{\prime}, \gamma_{0,2}^{\prime}\right)^{\prime}$ is the vector of coefficients of $x$ before exclusion restrictions on $x$ are imposed. Comparing (3.8) with (3.4),

$$
\pi_{1}^{*}=\Pi_{2}^{*} \alpha_{0}-\gamma_{0,1} \delta_{0}+\gamma_{0,2} .
$$

From (3.9), we see that the identification of the structural parameters $\alpha_{0}$, $\gamma_{0,1}$, and $\gamma_{0,2}$ requires restrictions on the structural equation (2.1). With cxclusion restrictions in (2.1), we have $\bar{\gamma}_{0}=J_{\gamma_{0}}$ and (3.9) becomes $\pi_{1}^{*}=$ $\left[\Pi_{2}^{*},\left(-\delta_{0}, I\right) J\right]\left(\alpha_{0}^{\prime}, \gamma_{0}^{\prime}\right)^{\prime}$. From this relation, we see that the rank identification of the structural parameters in $y=z \alpha_{0}+x J \gamma_{0}+u_{1}$ is that $\left[\Pi_{2}^{*},\left(-\delta_{o}, I\right) J\right]$ has full (column) rank. For the order identification condition, it is convenient to consider separately the two cases of exclusion restrictions of (1) $x_{(1)}$ appearing in (2.1) and (2) $x_{(1)}$ not appearing in (2.1). Consider first the case that $x_{(1)}$ is excluded from (2.1), which is equivalent to saying that $\gamma_{0,1}=0$. Without loss of generality, suppose that the first $k_{1}$ exogenous variables in $x_{(2)}$ are included but the remaining $K-1-k_{1}$ variables in $x_{(2)}$ are excluded from (2.1), i.e., $\gamma_{0,2}=$ $\left(\gamma_{0,21}^{\prime}, 0\right)^{\prime}$ where $\gamma_{0,21}$ is of dimension $k_{1}$. Conformably, let $\pi_{1}^{*}=\left(\pi_{11}^{*}, \pi_{12}^{* \prime}\right)^{\prime}$, $\Pi_{2}^{*}=\left(\Pi_{21}^{*}, \Pi_{22}^{* \prime}\right)^{\prime}$, and $\delta_{0}=\left(\delta_{0,1}^{\prime}, \delta_{0,2}^{\prime}\right)^{\prime}$. Since $\gamma_{0,1}=0,(3.9)$ is equivalent to

$$
\pi_{11}^{*}=\Pi_{21}^{*} \alpha_{0}+\gamma_{0,21}
$$

and

$$
\pi_{12}^{*}=\Pi_{22}^{*} \alpha_{0}
$$

It follows from (3.10) and (3.11) that the rank condition is equivalent to rank $\Pi_{22}^{*}=G_{1}$. The necessary order condition is $K-1-k_{1} \geqslant G_{1}$, i.e., the number of excluded variables in $x_{(2)}$ from the structural equation is greater than or equal 
to the number of endogenous variables on the right-hand side of (2.1). Consider next the case that $x_{(1)}$ is included in the structural equation. As in the previous case, suppose only the first $k_{1}$ exogenous variables in $x_{(2)}$ are included in (2.1). Eq. (3.9) is now equivalent to

$$
\pi_{11}^{*}=\Pi_{21}^{*} \alpha_{0}-\gamma_{0,1} \delta_{0,1}+\gamma_{0,21}
$$

and

$$
\pi_{12}^{*}=\Pi_{22}^{*} \alpha_{0}-\gamma_{0,1} \delta_{0,2} .
$$

The rank identification for this casc is $\operatorname{rank}\left[\Pi_{22}^{*},-\delta_{0,2}\right]=G_{1}+1$, and the necessary order condition is $K-1-k_{1} \geqslant G_{1}+1$. In any event, the identification condition is stronger than the identification condition for the classical simultaneous equation model. The exact identification of (2.1) for the classical model becomes underidentification for the semiparametric model. The order identification condition for the semiparametric model corresponds to the overidentification condition in the classical simultaneous equation model. The stronger condition for the identification of the semiparametric model is apparently due to the addition of a sample selection hias term of an unknown form in the bias corrected structural equation. Exogenous variables which are excluded from the structural equation (before bias correction), but appear in the selection bias term through the index $x \zeta_{0}$, identify the selection bias term. Intuitively, the included bias correction term introduces excluded exogenous variables back into the (bias) corrected structural equation, and the effective number of the included exogenous variables in this equation is the number of originally included exogenous variables plus one. Therefore, the order condition for identification requires stronger exclusion restrictions than the classical simultaneous equation model. For the parametric simultaneous equation sample selection model under normal disturbances, the bias correction term has a known nonlinear functional form, which introduces nonlinear restriction into the bias corrected structural equation. The nonlinear bias correction term with a particular known form due to normality helps identification even though the excluded exogenous variables are implicitly introduced back into the bias corrected structural equation. If the bias correction term in a parametric simultaneous equation sample selection model were a linear function of the index (Olsen, 1980), then stronger identification condition similar to the one of our semiparametric model would be needed. Putting it in another way, stronger identification condition is needed for our semiparametric model because it does not exclude the parametric specification of Olsen (1980).

The identification condition can be extended to the general model where $x \zeta$ represents a vector of indices. To distinguish the indices in $x \zeta$ for identification, each index is required to contain a relevant continuous exogenous variable which does not appear in the other indices (Ichimura and Lee, 1991). Suppose $x \zeta$ contains $m$ indices. With normalization, $x \zeta-\left(x_{(1)}+x_{(2)} \delta_{(1)}, \ldots\right.$, 
$\left.x_{(1 m)}+x_{(2)} \delta_{(m)}\right)$, where $x_{(11)}, \ldots, x_{(1 m)}$ are $m$ distinct continuous variables not contained in the subvector $x_{(2)}$. For this general model, $\delta=\left[\delta_{(1)}, \ldots, \delta_{(m)}\right]$ is now a matrix. The rank identification condition is that $\left[\Pi_{2}^{*},\left(-\delta_{0}, I\right) J\right]$ has full (column) rank.

\section{Semiparametric two-stage least squares estimation}

For the classical simultaneous equation model, the most popular IV method is the two-stage least squares method (2LS). A generalization of $2 \mathrm{LS}$ to the estimation of parametric simultaneous equation models with selectivity has been introduced in Lee, Maddala, and Trost (1980). The endogenous variables are regressed on all the exogenous variables and a sample selection bias term (inverse Mill's ratio) in the first stage. The regression predictors are then used as the instrumental variables in the second-stage estimation of the selection bias corrected structural equation.

For the estimation of the semiparametric model, define the following matrices:

$$
\begin{gathered}
\hat{X}_{2}=\left(\begin{array}{c}
t_{n}\left(x_{1} \hat{\zeta}, \hat{\xi}_{n}\right)\left(x_{(2) 1}-\mathrm{E}_{n}\left(x_{(2)} \mid x_{1} \hat{\zeta}\right)\right) \\
\vdots \\
t_{n}\left(x_{n} \hat{\zeta}, \hat{\xi}_{n}\right)\left(x_{(2) n}-\mathrm{E}_{n}\left(x_{(2)} \mid x_{n} \hat{\zeta}\right)\right)
\end{array}\right), \\
\hat{W}=\left(\begin{array}{c}
t_{n}\left(x_{1} \hat{\zeta}, \hat{\xi}_{n}\right)\left(w_{1}-\mathrm{E}_{n}\left(w \mid x_{1} \hat{\zeta}\right)\right) \\
\vdots \\
t_{n}\left(x_{n} \hat{\zeta}, \hat{\xi}_{n}\right)\left(w_{n}-\mathrm{E}_{n}\left(w \mid x_{n} \hat{\zeta}\right)\right)
\end{array}\right), \\
\hat{Y}=\left(\begin{array}{c}
t_{n}\left(x_{1} \hat{\zeta}, \hat{\xi}_{n}\right)\left(y_{1}-\mathrm{E}_{n}\left(y \mid x_{1} \hat{\zeta}\right)\right) \\
\vdots \\
t_{n}\left(x_{n} \hat{\zeta}, \hat{\xi}_{n}\right)\left(y_{n}-\mathrm{E}_{n}\left(y \mid x_{n} \hat{\zeta}\right)\right)
\end{array}\right),
\end{gathered}
$$

where $t_{n}\left(x \hat{\zeta}, \hat{\xi}_{n}\right)$ is a smooth quantile trimming function of $x \hat{\zeta}$ introduced in Sections A.2 and A.3 of the Appendix. The trimming function is differentiable with respect to its arguments $x \hat{\zeta}$ and $\hat{\xi}_{n}$ so that one can easily investigate the impact of the randomness of $\hat{\delta}$ and the sample quantiles $\hat{\xi}_{n}$ on the asymptotic properties of the derived estimators. A semiparametric two-stage least squares estimator (S2LS) is

$$
\hat{\beta}_{\mathrm{S} 2 \mathrm{LS}}=\left[\hat{W}^{\prime} \hat{X}_{2}\left(\hat{X}_{2}^{\prime} \hat{X}_{2}\right)^{-1} \hat{X}_{2}^{\prime} \hat{W}\right]^{-1} \hat{W}^{\prime} \hat{X}_{2}\left(\hat{X}_{2}^{\prime} \hat{X}_{2}\right)^{-1} \hat{X}_{2}^{\prime} \hat{Y}
$$

This estimator can be interpreted as being derived from a two-stage estimation procedure. In the first-stage estimation, the reduced form equations for $z$ in 
(3.7) are estimated by a semiparametric least squares method, and the predicted values for $z-\mathrm{E}_{n}(z \mid x \hat{\zeta})$ are used as instrumental variables for $z-\mathrm{E}_{n}(z \mid x \hat{\xi})$. In addition to $z$, if $x_{(1)}$ is included in $w$, an auxiliary reduced form equation for $x_{(1)}$ is also estimated by a semiparametric least squares method, and the predicted value for $x_{(1)}-\mathrm{E}_{n}\left(x_{(1)} \mid x_{\xi}\right)$ will be used as an instrumental variable for $x_{(1)}-\mathrm{E}_{n}\left(x_{(1)} \mid x \hat{\zeta}\right)$ for the estimation of (2.7). This two-stage estimator has a two-stage semiparametric least squares interpretation. To see these, define an auxiliary equation for $x_{(1)}$ :

$$
x_{(1)}=-x_{(2)} \delta_{0}+v_{1 * *},
$$

where $v_{1, *}=x \zeta_{0}$. Since $w^{*}=\left(z^{*}, x J\right),(3.5)$ and (4.2) imply that

$$
w^{*}=x_{(2)}\left[\Pi_{2}^{*},\left(-\delta_{0}, I\right) J\right]+v^{*},
$$

where $v^{*}=\left(v_{2}^{*},\left(v_{1, *}, 0\right) J\right)$, and conditional on $d^{*}>0$ and $x$,

$$
w=x_{(2)}\left[\Pi_{2}^{*},\left(-\delta_{0}, I\right) J\right]+\mathrm{E}\left(v^{*} \mid x, d^{*}>0\right)+v_{d},
$$

where $v_{d}=v^{*}-\mathrm{E}\left(v^{*} \mid x, d^{*}>0\right)$. Similarly, as in (2.7), since the distribution of $v^{*}$ is a function of $x_{\zeta_{0}}$,

$$
w_{i}-\mathrm{E}_{n}\left(w \mid x_{i} \hat{\zeta}\right)=\left(x_{(2) i}-\mathrm{E}_{n}\left(x_{(2)} \mid x_{i} \hat{\zeta}\right)\right) \Pi_{w}+\hat{v}_{n i}
$$

where $\Pi_{w}=\left[\Pi_{2}^{*},\left(-\delta_{0}, I\right) J\right]$. A semiparametric least squares (SLS) estimator of $\Pi_{w}$ with trimming function $t_{n}$ is $\hat{\Pi}_{w}=\left(\hat{X}_{2}^{\prime} \hat{X}_{2}\right)^{-1} \hat{X}_{2}^{\prime} \hat{W}$. It follows that $\hat{\beta}_{\mathrm{S} 2 \mathrm{LS}}=\left[\left(\hat{X}_{2} \hat{\Pi}_{w}\right)^{\prime}\left(\hat{X}_{2} \hat{\Pi}_{w}\right)\right]^{-1}\left(\hat{X}_{2} \hat{\Pi}_{w}\right)^{\prime} \hat{Y}$, which has a two-stage least squares interpretation. The regressor $x_{(1)}$ plays an interesting role in the estimation. This variable is exogenous in the equation system, however it behaves as if it were an endogenous variable in the estimation. It has been excluded from the list of regressors in the first-stage SLS estimation. This feature is compatible with the order identification condition in Section 3.

The propositions in Appendix Section A.4 can be used to derive the asymptotic properties of our estimator. Some of the detailed derivations will be referred to Appendix Section A.5. Since sample observations for (2.4) are available only after selection, all expectations will be taken as conditional expectations conditional on $d^{*}>0$. To simplify notation, the conditional argument $d^{*}>0$ will be suppressed. Thus $\mathrm{E}\left(\cdot \mid x \zeta_{0}\right)$ stands for $\mathrm{E}\left(\cdot \mid x \zeta_{0}, d^{*}>0\right)$ in subsequent presentation and the Appendix. Proposition 1 in Appendix Section A.4 implics that

$$
\begin{aligned}
\frac{1}{n} \hat{X}_{2}^{\prime} \hat{X}_{2} & =\frac{1}{n} \sum_{i=1}^{n} t_{n}\left(x_{i} \hat{\zeta}, \hat{\xi}_{n}\right)\left(x_{(2) i}-\mathrm{E}_{n}\left(x_{(2)} \mid x_{i} \hat{\zeta}\right)\right)^{\prime}\left(x_{(2) i}-\mathrm{E}_{n}\left(x_{(2)} \mid x_{i} \hat{\zeta}\right)\right) \\
& \stackrel{p}{\rightarrow} C,
\end{aligned}
$$


where

$$
C=\mathrm{E}\left[I_{T}\left(x \zeta_{0}\right)\left(x_{(2)}-\mathrm{E}\left(x_{(2)} \mid x \zeta_{0}\right)\right)^{\prime}\left(x_{(2)}-\mathrm{E}\left(x_{(2)} \mid x \zeta_{0}\right)\right)\right],
$$

and $I_{T}$ is the indicator function of $T$, where $T=\left[\xi_{p}\left(\delta_{0}\right), \xi_{(1-p)}\left(\delta_{0}\right)\right]$ with $\xi_{p}\left(\delta_{0}\right)$ and $\xi_{(1-p)}\left(\delta_{0}\right)$ being respectively the $p$ th and $(1-p)$ th quantiles of $x \zeta_{0}$. On the other hand, since, from $(4.2)^{\prime}, v^{*}=\left[v_{2}^{*},\left(x \zeta_{0}, 0\right) J\right]$ and $\mathrm{E}\left(v_{2}^{*} \mid x\right)=\mathrm{E}\left(v_{2}^{*} \mid x \zeta_{0}\right)$, it follows that $\mathrm{E}\left[v^{*}-\mathrm{E}\left(v^{*} \mid x \zeta_{n}\right) \mid x\right]=0$ and, by Proposition 1 in Appendix Section A.4,

$$
\begin{aligned}
\frac{1}{n} \hat{W}^{\prime} \hat{X}_{2}= & \frac{1}{n} \sum_{i=1}^{n} t_{n}\left(x_{i} \hat{\zeta}, \hat{\xi}_{n}\right)\left(w_{i}-\mathrm{E}_{n}\left(w \mid x_{i} \hat{\zeta}\right)\right)^{\prime}\left(x_{(2) i}-\mathrm{E}_{n}\left(x_{(2)} \mid x_{i} \hat{\zeta}\right)\right) \\
= & \Pi_{w}^{\prime} \frac{1}{n} \sum_{i=1}^{n} t_{n}\left(x_{i} \hat{\zeta}, \hat{\xi}_{n}\right)\left(x_{(2) i}-\mathrm{E}_{n}\left(x_{(2)} \mid x_{i} \hat{\zeta}\right)\right)^{\prime}\left(x_{(2) i}-\mathrm{E}_{n}\left(x_{(2)} \mid x_{i} \hat{\zeta}\right)\right) \\
& +\frac{1}{n} \sum_{i=1}^{n} t_{n}\left(x_{i} \hat{\zeta}, \hat{\xi}_{n}\right)\left(v_{i}^{*}-\mathrm{E}_{n}\left(v^{*} \mid x_{i} \hat{\zeta}\right)\right)^{\prime}\left(x_{(2) i}-\mathrm{E}_{n}\left(x_{(2)} \mid x_{i} \hat{\zeta}\right)\right) \\
& \stackrel{p}{\rightarrow} \Pi_{w}^{\prime} C .
\end{aligned}
$$

It follows that under the assumption [Assumption 1(6)] that $C$ is nonsingular,

$$
\frac{1}{n} \hat{W}^{\prime} \hat{X}_{2}\left(\hat{X}_{2}^{\prime} \hat{X}_{2}\right)^{-1} \hat{X}_{2}^{\prime} \hat{W} \stackrel{p}{\rightarrow} \Pi_{w}^{\prime} C \Pi_{w} .
$$

The rank identification condition that $\Pi_{w}$ has full column rank is necessary for the limiting matrix in (4.8) to be nonsingular. Let $\hat{U}_{n}=\left(t_{n}\left(x_{1} \hat{\zeta}, \hat{\xi}_{n}\right) \hat{u}_{n 1}, \cdots\right.$, $\left.t_{n}\left(x_{n}, \hat{\zeta}, \hat{\xi}_{n}\right) \hat{u}_{n n}\right)^{\prime}$. Eqs. (2.7) and (4.1) imply that

$$
\hat{\beta}_{\mathrm{S} 2 \mathrm{LS}}-\beta_{0}=\left[\hat{W}^{\prime} \hat{X}_{2}\left(\hat{X}_{2}^{\prime} \hat{X}_{2}\right)^{-1} \hat{X}_{2}^{\prime} \hat{W}\right]^{-1} \hat{W}^{\prime} \hat{X}_{2}\left(\hat{X}_{2}^{\prime} \hat{X}_{2}\right)^{-1} \hat{X}_{2}^{\prime} \hat{U}_{n} \text {. }
$$

Since $\operatorname{plim}_{n \rightarrow \infty}(1 / n) \hat{X}_{2}^{\prime} \hat{U}_{n}=\mathrm{E}\left(I_{T}\left(x \zeta_{0}\right)\left[x_{(2)}-\mathrm{E}\left(x_{(2)} \mid x \zeta_{0}\right)\right]^{\prime}\left[u_{1}-\mathrm{E}\left(u_{1} \mid x \zeta_{0}\right)\right]\right)=0$, $\hat{\beta}_{\mathrm{S} 2 \mathrm{LS}}$ is a consistent estimator of $\beta_{0}$.

The asymptotic distribution of $\hat{\beta}_{\mathrm{S} 2 \mathrm{LS}}$ can be derived from (4.9). Let $\omega\left(x_{i} \zeta_{0}, \delta_{0}\right)$ denote the conditional variance of $u_{d i}$. Let $\nabla \mathrm{E}\left(u_{1} \mid x \zeta_{0}\right)$ denote the first-order derivative of $\mathrm{E}\left(u_{1} \mid x \zeta_{0}\right)$ with respect to the argument $x \zeta_{0}$. As shown in (A.5.1) of the Appendix,

$$
\begin{aligned}
\frac{1}{\sqrt{n}} \hat{X}_{2}^{\prime} \hat{U}_{n}= & \frac{1}{\sqrt{n}} \sum_{i=1}^{n} t_{n}\left(x_{i} \hat{\zeta}, \hat{\xi}_{n}\right)\left(x_{(2) i}-\mathrm{E}_{n}\left(x_{(2)} \mid x_{i} \hat{\zeta}\right)\right)^{\prime}\left(u_{1 i}-\mathrm{E}_{n}\left(u_{1} \mid x_{i} \hat{\zeta}\right)\right) \\
= & \frac{1}{\sqrt{n}} \sum_{i=1}^{n} t_{n}\left(x_{i} \zeta_{0}, \xi_{0}\right)\left(x_{(2) i}-\mathrm{E}\left(x_{(2) i} \mid x_{i} \zeta_{0}\right)\right)^{\prime}\left(u_{1 i}-\mathrm{E}\left(u_{1 i} \mid x_{i} \zeta_{0}\right)\right) \\
& -\mathrm{E}\left(I_{T}\left(x \zeta_{0}\right)\left(x_{(2)}-\mathrm{E}\left(x_{(2)} \mid x \zeta_{0}\right)\right)^{\prime}\left(x_{(2)}-\mathrm{E}\left(x_{(2)} \mid x \zeta_{0}\right)\right)\right. \\
& \left.\times \nabla \mathrm{E}\left(u_{1} \mid x \zeta_{0}\right)\right) \sqrt{n}\left(\hat{\delta}-\delta_{0}\right)+o_{p}(1) .
\end{aligned}
$$


The first term on the right-hand side of (4.10) captures the impact of the disturbance $u_{d}$ in the structural equation (2.4) on the limiting distribution of $\hat{\beta}_{\mathrm{S} 2 \mathrm{LS}}$, and the second component captures the randomness of the firststage estimator $\hat{\delta}$ of $\delta_{0}$ on the limiting distribution of $\hat{\beta}_{\mathrm{s} 2 \mathrm{LS}}$. Under the assumption [Assumption 1(4) in the Appendix] that $\sqrt{n}\left(\hat{\delta}-\delta_{0}\right)$ is asymptotically normal, $\mathrm{N}\left(0, V_{\delta}\right)$, and is asymptotically uncorrelated with $u_{1}-\mathrm{E}\left(u_{1} \mid x \zeta_{0}\right)$, the Lindeberg Feller and multivariate central limit theorems imply that

$$
\frac{1}{\sqrt{n}} \hat{X}_{2}^{\prime} \hat{U}_{n} \stackrel{D}{\rightarrow} \mathrm{N}(0, \Delta)
$$

where

$$
\Delta=\mathrm{E}\left[I_{T}\left(x \zeta_{0}\right)\left(x_{(2)}-\mathrm{E}\left(x_{(2)} \mid x \zeta_{0}\right)\right)^{\prime} \omega\left(x \zeta_{0}, \delta_{0}\right)\left(x_{(2)}-\mathrm{E}\left(x_{(2)} \mid x \zeta_{0}\right)\right)\right]+D V_{\delta} D^{\prime}
$$

and

$$
D=\mathrm{E}\left[I_{T}\left(x \zeta_{0}\right)\left(x_{(2)}-\mathrm{E}\left(x_{(2)} \mid x \zeta_{0}\right)\right)^{\prime}\left(x_{(2)}-\mathrm{E}\left(x_{(2)} \mid x \zeta_{0}\right)\right) \nabla \mathrm{E}\left(u_{1} \mid x \zeta_{0}\right)\right] .
$$

The above assumption [Assumption 1(4)] for the first-stage estimator will be satisfied with parametric or semiparametric estimators of discrete choice models such as the probit or logit estimators (under correct distributional assumptions), the Ichimura single-index estimator (Ichimura, 1987), and the semiparametric maximum likelihood estimator of Klein and Spady (1987) for binary choice models, and the multiple index estimator in Ichimura and Lee (1991) for polychotomous choice models. In general, for a choice model with $L$ alternatives and $T$ sample observations where $T>n$, if

$$
\sqrt{n}\left(\hat{\delta}-\delta_{0}\right)=\frac{1}{\sqrt{T}} \sum_{i=1}^{T} f\left(x_{i}, I_{1, i}, \ldots, I_{L, i}\right)+o_{p}(1),
$$

where $I_{l}$ is a dichotomous choice indicator for the $l$ alternative and $f\left(x, I_{1}, \ldots, I_{L}\right)$ is some measurable function with zero mean, is asymptotically normal, Assumption 1(4) will be satisfied. This is so, because

$$
\begin{aligned}
\mathrm{E}^{*} & \left\{\sum_{j=1}^{T} f\left(x_{j}, I_{1, j}, \ldots, I_{L, j}\right)\left(u_{1 i}-\mathrm{E}\left(u_{1 i} \mid x_{i} \zeta_{0}\right)\right)\right\} \\
& =\mathrm{E}^{*}\left\{f\left(x_{i}, I_{1, i}, \ldots, I_{L, i}\right)\left(u_{1 i}-\mathrm{E}\left(u_{1 i} \mid x_{i} \zeta_{0}\right)\right)\right\} \\
& =\mathrm{E}^{*}\left\{\mathrm{E}\left[u_{1 i}-\mathrm{E}\left(u_{1 i} \mid x_{i} \zeta_{0}\right) \mid x_{i}, I_{1 i}=1\right] f\left(x_{i}, I_{1, i}, \ldots, I_{L, i}\right)\right\}, \\
& =0
\end{aligned}
$$


where $E^{*}$ denotes the unconditional expectation taken with the whole population (not just the subpopulation with $d^{*}>0$ ).

In conclusion,

$$
\sqrt{n}\left(\hat{\beta}_{\mathrm{S} 2 \mathrm{LS}}-\beta_{0}\right) \stackrel{D}{\rightarrow} \mathrm{N}(0, \Omega)
$$

where

$$
\Omega=\left(\Pi_{w}^{\prime} C \Pi_{w}\right)^{-1} \Pi_{w}^{\prime} \Delta \Pi_{w}\left(\Pi_{w}^{\prime} C \Pi_{w}\right)^{-1} .
$$

\section{Semiparametric generalized two-stage least squares estimation}

The S2LS in (4.1) is simple, but it is not an efficient IV estimator for the estimation of (2.7) because it has not incorporated the complicated covariances structure of $\hat{u}_{n i}$ in estimation.

The disturbance $\hat{u}_{n i}$ in (2.7) can be decomposed into three components:

$$
\begin{aligned}
\hat{u}_{n i}= & u_{1 i}-\mathrm{E}_{n}\left(u_{1} \mid x_{i} \hat{\zeta}\right) \\
= & \left(u_{1 i}-\mathrm{E}\left(u_{1 i} \mid x_{i} \zeta_{0}\right)\right)-\left(\mathrm{E}_{n}\left(u_{1} \mid x_{i} \hat{\zeta}\right)-\mathrm{E}_{n}\left(u_{1} \mid x_{i} \zeta_{0}\right)\right) \\
& -\left(\mathrm{E}_{n}\left(u_{1} \mid x_{i} \zeta_{0}\right)-\mathrm{E}\left(u_{1 i} \mid x_{i} \zeta_{0}\right)\right) .
\end{aligned}
$$

The first component represents the disturbance $u_{d i}$ in the structural equation (2.4) after the correction of selection bias. The second component represents the disturbance introduced in $\mathrm{E}_{n}\left(u_{1} \mid x_{i} \zeta_{0}\right)$ by replacing $\zeta_{0}$ by the estimate $\hat{\zeta}$. In the parametric two-stage estimation of the sample selection model with a discrete choice decision rule, these two components of the disturbance are asymptotically uncorrelated (Heckman, 1979; Lee, Maddala, and Trost, 1980). ${ }^{1}$ This is also the case for the semiparametric model in (2.1) and (2.3). The last component represents the error introduced by the nonparametric estimate of the conditional expectation of $u_{1 i}$. Even though the last component has a rather complicated structure, it does not influence the asymptotic distribution of $\hat{\beta}_{\mathrm{S} 2 \mathrm{LS}}$, due to an asymptotic orthogonality property of the index model (see the Appendix for details, in particular, Propositions 4 and 6 of Section A.4).

\footnotetext{
${ }^{1}$ For the sample selection model with a tobit type decision rule, if the first-stage estimate is a tobit MLE, the two components can be correlated (see Lee et al., 1980)].
} 
The variance of $u_{d i}$ in (2.4) is a function of $x_{i} \zeta_{0}$. It can be estimated nonparametrically. Let $H$ be a kernel function with a bandwidth $b_{n}{ }^{2}$ Define

$$
\begin{aligned}
& \omega_{n i}(\hat{\beta}, \hat{\delta})= \\
& \frac{\sum_{j \neq i}^{n}\left(y_{j}-w_{j} \hat{\beta}\right)^{2} H\left(\frac{x_{i} \hat{\zeta}-x_{j} \hat{\zeta}}{b_{n}}\right)}{\sum_{j \neq i}^{n} H\left(\frac{x_{i} \hat{\zeta}-x_{j} \hat{\zeta}}{b_{n}}\right)}-\left[\frac{\sum_{j \neq i}^{n}\left(y_{j}-w_{j} \hat{\beta}\right) H\left(\frac{x_{i} \hat{\zeta}-x_{j} \hat{\zeta}}{b_{n}}\right)}{\sum_{j \neq i}^{n} H\left(\frac{x_{i} \hat{\zeta}-x_{j} \hat{\zeta}}{b_{n}}\right)}\right],
\end{aligned}
$$

where $\hat{\beta}$ is an initial consistent estimate of $\beta$ [c.g., $\hat{\beta}_{\mathrm{S} 2 \mathrm{LS}}$ in (4.1)], $\hat{\omega}_{n}(\hat{\beta}, \hat{\delta})$ provides a nonparametric estimate of the variance $\omega\left(x_{i} \zeta_{0}, \delta_{0}\right)$ of $u_{d i}$ at $x_{i} \zeta_{0}$. The above arguments suggest the following covariance matrix:

$$
\hat{\Sigma}=\hat{A}_{n}+\frac{\partial \mathrm{E}_{n}(\hat{\beta}, \hat{\zeta})}{\partial \delta^{\prime}} \hat{V}_{n . \delta} \frac{\partial \mathrm{E}_{n}^{\prime}(\hat{\beta}, \hat{\zeta})}{\partial \delta},
$$

where $\hat{\Lambda}_{n}$ is a diagonal matrix with diagonal elements $\hat{\omega}_{n i}(\hat{\beta}, \hat{\delta}), i=1, \ldots, n$, $\hat{V}_{n, \delta} / n$ is a consistent estimate of the limiting covariance matrix $V_{\delta}$ of $\sqrt{n}\left(\hat{\delta}-\delta_{0}\right)$, and

$$
\begin{aligned}
\frac{\partial \mathrm{E}_{n}^{\prime}(\hat{\beta}, \hat{\zeta})}{\partial \delta}= & {\left[t_{n}\left(x_{1} \hat{\zeta}, \hat{\xi_{n}}\right) \frac{\partial \mathrm{E}_{n}\left(y-w \hat{\beta} \mid x_{1} \hat{\zeta}\right)}{\partial \delta}, \ldots,\right.} \\
& \left.t_{n}\left(x_{n} \hat{\zeta}, \hat{\xi}_{n}\right) \frac{\partial \mathrm{E}_{n}\left(y-w \hat{\beta} \mid x_{n} \hat{\zeta}\right)}{\partial \delta}\right] .
\end{aligned}
$$

By a formula of inversion of a partitioned matrix, the inverse of $\hat{V}_{n, \delta}$ involves only inversion of matrices of the dimension of $\delta$ :

$$
\begin{aligned}
\hat{\Sigma}^{-1}= & \hat{\Lambda}_{n}^{-1}-\hat{\Lambda}_{n}^{-1} \frac{\partial \mathrm{E}_{n}(\hat{\beta}, \hat{\zeta})}{\partial \delta^{\prime}}\left(\hat{V}_{n, \delta}^{-1}+\frac{\partial \mathrm{E}_{n}^{\prime}(\hat{\beta}, \hat{\zeta})}{\partial \delta} \hat{\Lambda}_{n}^{-1} \frac{\partial \mathrm{E}_{n}(\hat{\beta}, \hat{\zeta})}{\partial \delta^{\prime}}\right)^{-1} \\
& \times \frac{\partial \mathrm{E}_{n}^{\prime}(\hat{\beta}, \hat{\zeta})}{\partial \delta} \hat{\Lambda}_{n}^{-1}
\end{aligned}
$$

For the parametric simultaneous equation sample selection model, several generalized two-stage least squares estimators (G2I S) have been introduced (Lee, 1981; Amemiya, 1983). For the semiparametric model, the following estimator is a semiparametric generalized two-stage least squares estimator (SG2LS):

$$
\hat{\beta}_{\mathrm{SG}}=\left[\hat{W}^{\prime} \hat{X}_{2}\left(\hat{X}_{2}^{\prime} \hat{X}_{2}\right)^{-1} \hat{X}_{2}^{\prime} \hat{\Sigma}^{-1} \hat{W}\right]^{-1} \hat{W}^{\prime} \hat{X}_{2}\left(\hat{X}_{2}^{\prime} \hat{X}_{2}\right)^{-1} \hat{X}_{2}^{\prime} \hat{\Sigma}^{-1} \hat{Y} \text {. }
$$

\footnotetext{
${ }^{2}$ The kernel $K$ with $a_{n}$ in (2.6) can be used. However, it is desirable to use a separate kernel function so as to avoid unnecessary stronger requirements on the rate of convergence for bandwidth parameters.
} 
An alternative SG2LS estimator is

$$
\begin{aligned}
\hat{\beta}_{\mathrm{SG}}= & {\left[\hat{W}^{\prime} \hat{\Sigma}^{-1} \hat{X}_{2}\left(\hat{X}_{2}^{\prime} \hat{\Sigma}^{-1} \hat{X}_{2}\right)^{-1} \hat{X}_{2}^{\prime} \hat{\Sigma}^{-1} \hat{W}\right]^{-1} } \\
& \times \hat{W}^{\prime} \hat{\Sigma}^{-1} \hat{X}_{2}\left(\hat{X}_{2}^{\prime} \hat{\Sigma}^{-1} \hat{X}_{2}\right)^{-1} \hat{X}_{2}^{\prime} \hat{\Sigma}^{-1} \hat{Y} .
\end{aligned}
$$

It can be shown that these two estimators have the same asymptotic distribution. ${ }^{3}$ The computation of $\hat{\beta}_{\mathrm{SG}}$ is simpler, but the data transformations in $\hat{\beta}_{\mathrm{SG}}$ are intuitively appealing.

Substituting (2.7) into (5.6) and (5.7),

$$
\begin{aligned}
\hat{\beta}_{\mathrm{SG}}-\beta_{0}= & {\left[\hat{W}^{\prime} \hat{X}_{2}\left(\hat{X}_{2}^{\prime} \hat{X}_{2}\right)^{-1} \hat{X}_{2}^{\prime} \hat{\Sigma}^{-1} \hat{W}\right]^{-1} } \\
& \times \hat{W}^{\prime} \hat{X}_{2}\left(\hat{X}_{2}^{\prime} \hat{X}_{2}\right)^{-1} \hat{X}_{2}^{\prime} \hat{\Sigma}^{-1} \hat{U}_{n}
\end{aligned}
$$

and

$$
\begin{aligned}
\tilde{\beta}_{\mathrm{SG}}-\beta_{0}= & {\left[\hat{W}^{\prime} \hat{\Sigma}^{-1} \hat{X}_{2}\left(\hat{X}_{2}^{\prime} \hat{\Sigma}^{-1} \hat{X}_{2}\right)^{-1} \hat{X}_{2}^{\prime} \hat{\Sigma}^{-1} \hat{W}\right]^{-1} } \\
& \times \hat{W}^{\prime} \hat{\Sigma}^{-1} \hat{X}_{2}\left(\hat{X}_{2}^{\prime} \hat{\Sigma}^{-1} \hat{X}_{2}\right)^{-1} \hat{X}_{2}^{\prime} \hat{\Sigma}^{-1} \hat{U}_{n}
\end{aligned}
$$

Proposition 1 of Appendix Section A.4 implies that

$$
\begin{aligned}
\frac{1}{n} \hat{X}_{2}^{\prime} \hat{A}_{n}^{-1} \hat{X}_{2}= & \frac{1}{n} \sum_{i=1}^{n} t_{n}\left(x_{i} \hat{\zeta}, \hat{\xi}_{n}\right)\left(x_{(2) i}-\mathrm{E}_{n}\left(x_{(2)} \mid x_{i} \hat{\zeta}\right)\right)^{\prime} \\
& \times \omega_{n i}^{-1}(\hat{\beta}, \hat{\delta})\left(x_{(2) i}-\mathrm{E}_{n}\left(x_{(2)} \mid x_{i} \hat{\zeta}\right)\right) \\
& { }^{p}, C_{\omega},
\end{aligned}
$$

where

$$
C_{\omega}=\mathrm{E}\left[I_{T}\left(x \zeta_{0}\right)\left(x_{(2)}-\mathrm{E}\left(x_{(2)} \mid x \zeta_{0}\right)\right)^{\prime} \omega^{-1}\left(x \zeta_{0}, \delta_{0}\right)\left(x_{(2)}-\mathrm{E}\left(x_{(2)} \mid x \zeta_{0}\right)\right)\right]
$$

and

$$
\begin{aligned}
\frac{1}{n} \hat{X}_{2}^{\prime} \hat{\Lambda}_{n}^{-1} \hat{W} & =\frac{1}{n} \sum_{i=1}^{n} t_{n}\left(x_{i} \hat{\zeta}, \hat{\xi}_{n}\right)\left(x_{2 i}-\mathrm{E}_{n}\left(x_{(2)} \mid x_{i} \hat{\zeta}\right)\right)^{\prime} \omega_{n i}^{-1}(\hat{\beta}, \hat{\delta})\left(w_{i}-\mathrm{E}_{n}\left(w \mid x_{i} \hat{\zeta}\right)\right) \\
& \stackrel{p}{\rightarrow} C_{\omega} \Pi_{w} .
\end{aligned}
$$

Furthermore, let $\partial \mathrm{E}\left(u_{1} \mid x \zeta_{0}, \delta_{0}\right) / \partial \delta$ denote $\partial \mathrm{E}\left(u_{1} \mid x \zeta\right) /\left.\partial \delta\right|_{\delta=\delta_{0}}$. An explicit expression for this derivative [Appendix Eq. (A.4.11)] is

$$
\frac{\partial \mathrm{E}\left(u_{1} \mid x \zeta_{0}, \delta_{0}\right)}{\partial \delta}=\left(x_{(2)}-\mathrm{E}\left(x_{(2)} \mid x \zeta_{0}\right)\right)^{\prime} \nabla \mathrm{E}\left(u_{1} \mid x \zeta_{0}\right) \text {. }
$$

\footnotetext{
${ }^{3}$ For the parametric sample selection model, the asymptotic equivalency between two such similar estimators has been shown in Lee (1981) and Amemiya (1983).
} 
Proposition 1 implies that

$$
\frac{1}{n} \hat{X}_{2}^{\prime} \hat{\Lambda}_{n}^{-1} \frac{\partial \mathrm{E}_{n}(\hat{\beta}, \hat{\zeta})}{\partial \delta^{\prime}} \stackrel{p}{\rightarrow} D_{\omega},
$$

where

$$
D_{\omega}=\mathrm{E}\left[I_{T}\left(x \zeta_{0}\right)\left(x_{(2)}-\mathrm{E}\left(x_{(2)} \mid x \zeta_{0}\right)\right)^{\prime} \omega^{-1}\left(x \zeta_{0}, \delta_{0}\right) \frac{\partial \mathrm{E}\left(u_{1} \mid x \zeta_{0}, \delta_{0}\right)}{\partial \delta^{\prime}}\right]
$$

and

$$
\frac{1}{n} \frac{\partial \mathrm{E}_{n}(\hat{\beta}, \hat{\zeta})}{\partial \delta} \hat{A}_{n}^{-1} \frac{\partial \mathrm{E}_{n}(\hat{\beta}, \hat{\zeta})}{\partial \delta^{\prime}} \stackrel{p}{\rightarrow} E_{\omega}
$$

where

$$
\mathrm{E}_{\omega}=\mathrm{E}\left[I_{T}\left(x \zeta_{0}\right) \frac{\partial \mathrm{E}\left(u_{1} \mid x \zeta_{0}, \delta_{0}\right)}{\sqrt{\partial \delta}} \omega^{-1}\left(x \zeta_{0}, \delta_{0}\right) \frac{\partial \mathrm{E}\left(u_{1} \mid x \zeta_{0}, \delta_{0}\right)}{\partial \delta^{\prime}}\right] .
$$

Similarly,

$$
\frac{1}{n} \frac{\partial \mathrm{E}_{n}(\hat{\beta}, \hat{\zeta})}{\partial \delta} \hat{A}_{n}^{-1} \hat{W}_{n} \stackrel{p}{\rightarrow} D_{\omega}^{\prime} \Pi_{w} .
$$

With (5.5), by combining the above relations,

$$
\frac{1}{n} \hat{X}_{2}^{\prime} \hat{\Sigma}^{-1} \hat{X}_{2} \stackrel{p}{\rightarrow} \Gamma
$$

and

$$
\frac{1}{n} \hat{X}_{2}^{\prime} \hat{\Sigma}^{-1} \hat{W} \stackrel{p}{\rightarrow} \Gamma \Pi_{w}
$$

where

$$
\Gamma=C_{\omega}-D_{\omega}\left(V_{\delta}{ }^{1}+E_{\omega}\right){ }^{1} D_{\omega}^{\prime} .
$$

Furthermore, from the derivations in Appendix Section A.5 [Eq. (A.5.2)],

$$
\frac{1}{\sqrt{n}} \hat{X}_{2}^{\prime} \hat{\Sigma}^{-1} \hat{U}_{n} \stackrel{D}{\rightarrow} \mathrm{N}(0, \Gamma) .
$$

Eqs. (5.9)-(5.13) imply that

$$
\sqrt{n}\left(\tilde{\beta}_{\mathrm{SG}}-\beta_{0}\right) \stackrel{D}{\rightarrow} \mathrm{N}\left(0,\left(\Pi_{w}^{\prime} \Gamma \Pi_{w}\right)^{-1}\right)
$$


From (4.5), (4.7), (5.10), and (5.11), both $\hat{W}^{\prime} \hat{X}_{2}\left(\hat{X}_{2}^{\prime} \hat{X}_{2}\right)^{-1}$ and $\hat{W}^{\prime} \hat{\Sigma}^{-1} \hat{X}_{2}\left(\hat{X}_{2}^{\prime} \hat{\Sigma}^{-1} \hat{X}_{2}\right)^{-1}$ converge in probability to $\Pi_{w}^{\prime}$. Hence $\sqrt{n}\left(\hat{\beta}_{\mathrm{SG}}-\beta_{0}\right)$ has the same limiting distribution of $\sqrt{n}\left(\tilde{\beta}_{\mathrm{SG}}-\beta_{0}\right)$. The asymptotic covariance matrix of $\hat{\beta}_{\mathrm{SG}}$ (or $\tilde{\beta}_{\mathrm{SG}}$ ) can be consistently estimated by

$$
\hat{\Omega}_{\mathrm{G}, n}=\left[\hat{W}^{\prime} \hat{X}_{2}\left(\hat{X}_{2}^{\prime} \hat{X}_{2}\right)^{-1} \hat{X}_{2}^{\prime} \hat{\Sigma}^{-1} \hat{W}\right]^{-1}
$$

or

$$
\tilde{\Omega}_{\mathrm{G}, n}=\left[\hat{W}^{\prime} \hat{\Sigma}^{-1} \hat{X}_{2}\left(\hat{X}_{2}^{\prime} \hat{\Sigma}^{-1} \hat{X}_{2}\right)^{-1} \hat{X}_{2}^{\prime} \hat{\Sigma}^{-1} \hat{W}\right]^{-1} .
$$

Let $\hat{Q}=\hat{X}_{2}\left(\hat{X}_{2}^{\prime} \hat{X}_{2}\right)^{-1} \hat{X}_{2}^{\prime} \hat{W}$. By the generalized Schwartz inequality, $\hat{X}_{2}^{\prime} \hat{\Sigma}^{-1} \hat{X}_{2} \geqslant \hat{X}_{2}^{\prime} \hat{Q}\left(\hat{Q}^{\prime} \hat{\Sigma} \hat{Q}\right)^{-1} \hat{Q}^{\prime} \hat{X}_{2}$. Hence

$$
\begin{aligned}
\Pi_{w}^{\prime} \Gamma \Pi_{w} & =\Pi_{w}^{\prime} \operatorname{plim} \frac{1}{n} \hat{X}_{2}^{\prime} \hat{\Sigma}^{-1} \hat{X}_{2} \Pi_{w} \\
& \geqslant \Pi_{w}^{\prime} \operatorname{plim} \frac{1}{n} \hat{X}_{2}^{\prime} \hat{Q}\left(\hat{Q}^{\prime} \hat{\Sigma} \hat{Q}\right)^{-1} \hat{Q}^{\prime} \hat{X}_{2} \Pi_{w} \\
& =\Pi_{w}^{\prime} \operatorname{plim} \frac{1}{n} \hat{X}_{2}^{\prime} \hat{W}\left(\hat{\Pi}_{w}^{\prime} \hat{X}_{2}^{\prime} \hat{\Sigma} \hat{X}_{2} \hat{\Pi}_{w}\right){ }^{1} \hat{W}^{\prime} \hat{X}_{2} \Pi_{w} \\
& =\Omega^{-1}
\end{aligned}
$$

by (4.7), (4.14), and the fact that $(1 / n) \hat{X}_{2}^{\prime} \hat{\Sigma} \hat{X}_{2} \stackrel{p}{\rightarrow} \Delta$. Hence $\tilde{\beta}_{\mathrm{SG}}$ is asymptotically efficient relative to $\hat{\beta}_{\mathrm{S} 2 \mathrm{LS}}$.

The SG2LS estimators $\hat{\beta}_{\mathrm{SG}}$ and $\tilde{\beta}_{\mathrm{SG}}$ are not only asymptotically efficient relative to the S2LS estimator $\hat{\beta}_{\mathrm{S} 2 \mathrm{LS}}$. They have also an asymptotically optimal property. They are asymptotically efficient IV estimators for the estimation of (2.7) (conditional on the choice of first-stage estimator of $\delta_{0}$ and the same trimmed version). Let $p_{i}$ be an instrumental variable for $w_{i}$ and $\hat{\beta}_{p}$ be the IV estimator:

$$
\hat{\beta}_{p}=\left(P^{\prime} \hat{W}\right)^{-1} P^{\prime} \hat{Y}
$$

where

$$
P=\left[t_{n}\left(x_{1} \hat{\zeta}, \hat{\xi}_{n}\right) p_{1}^{\prime}, \ldots, t_{n}\left(x_{n} \hat{\zeta}, \hat{\xi}_{n}\right) p_{n}^{\prime}\right]^{\prime}
$$

Eq. (2.7) implies that

$$
\hat{\beta}_{p}-\beta_{0}=\left(P^{\prime} \hat{W}\right)^{-1} P^{\prime} \hat{U}_{n} .
$$


For this case, the component $\mathrm{E}_{n}\left(u_{1} \mid x_{i} \zeta_{0}\right)-\mathrm{E}\left(u_{1} \mid x_{\zeta_{0}}=x_{i} \zeta_{0}\right)$ in $\hat{u}_{n i}$ has a significant impact on the asymptotic distribution of $\hat{\beta}_{p}$. $F$ rom (A.S.4) of the Appendix,

$$
\begin{aligned}
& \frac{1}{\sqrt{n}} \sum_{i=1}^{n} t_{n}\left(x_{i} \zeta_{0}, \zeta_{0}\right) p_{i}^{\prime}\left(u_{1 i}-\mathrm{E}_{n}\left(u_{1} \mid x_{i} \zeta_{0}\right)\right) \\
& \stackrel{D}{=}{ }_{\sqrt{n}}^{1} \sum_{i=1}^{n} I_{T}\left(x_{i} \zeta_{0}\right)\left(p_{i}-\mathrm{E}\left(p_{i} \mid x_{i} \zeta_{0}\right)\right)^{\prime}\left(u_{1 i}-\mathrm{E}\left(u_{1 i} \mid x_{i} \zeta_{0}\right)\right),
\end{aligned}
$$

where $\stackrel{D}{=}$ means that both sides have the same limiting distribution. Similar to (4.7),

$$
\begin{aligned}
\frac{1}{n} P^{\prime} \hat{W} & \stackrel{p}{\rightarrow} \mathrm{E}\left(I_{T}\left(x \zeta_{0}\right) p^{\prime}\left(x_{(2)}-\mathrm{E}\left(x_{(2)} \mid x \zeta_{0}\right)\right) \Pi_{w}\right. \\
& =\mathrm{E}\left(I_{T}\left(x \zeta_{0}\right)\left(p-\mathrm{E}\left(p \mid x \zeta_{0}\right)\right)^{\prime}\left(x_{(2)}-\mathrm{E}\left(x_{(2)} \mid x \zeta_{0}\right)\right) \Pi_{w^{\prime}} .\right.
\end{aligned}
$$

The latter equality in (5.22) holds because $I_{T}\left(x \zeta_{0}\right)$ is a function of $x \zeta_{0}$ alone. From (A.5.3) and (A.5.4) in Appendix Section A.5,

$$
\frac{1}{\sqrt{n}} P^{\prime} \hat{U}_{n} \stackrel{D}{\rightarrow} \mathrm{N}\left(0, \Delta_{p}\right)
$$

where

$$
\begin{aligned}
\Delta_{p}= & \mathrm{E}\left[I_{T}\left(x \zeta_{0}\right)\left(p-\mathrm{E}\left(p \mid x \zeta_{0}\right)\right)^{\prime} \omega\left(x \check{\zeta}_{0}, \delta_{0}\right)\left(p-\mathrm{E}\left(p \mid x \zeta_{0}\right)\right)\right] \\
& +\mathrm{E}\left[I_{T}\left(x \zeta_{0}\right)\left(p-\mathrm{E}\left(p \mid x \zeta_{0}\right)\right)^{\prime}\left(x_{(2)}-\mathrm{E}\left(x_{(2)} \mid x \zeta_{0}\right)\right) \nabla \mathrm{E}\left(u_{1} \mid x \zeta_{0}\right)\right] \\
& \times V_{\delta} \mathrm{E}\left[I_{T}\left(x \zeta_{0}\right)\left(p-\mathrm{E}\left(p \mid x \zeta_{0}\right)\right)^{\prime}\left(x_{(2)}-\mathrm{E}\left(x_{(2)} \mid x \zeta_{0}\right) \nabla \mathrm{E}\left(u_{1} \mid x \zeta_{0}\right)\right]^{\prime} .\right.
\end{aligned}
$$

It follows that

$$
\sqrt{n}\left(\hat{\beta}_{p}-\beta\right) \stackrel{D}{\rightarrow} \mathrm{N}\left(0, \Omega_{p}\right)
$$

where

$$
\begin{aligned}
\Omega_{p}= & \left\{\mathrm{E}\left[I_{T}\left(x \zeta_{0}\right)\left(p-\mathrm{E}\left(p \mid x \zeta_{0}\right)\right)^{\prime}\left(x_{(2)}-\mathrm{E}\left(x_{(2)} \mid x \zeta_{0}\right)\right) \Pi_{w}\right\}^{-1}\right. \\
& \times \Delta_{p}\left\{\mathrm{E}\left(I_{T}\left(x \zeta_{0}\right)\left(p-\mathrm{E}\left(p \mid x \zeta_{0}\right)\right)^{\prime}\left(x_{(2)}-\mathrm{E}\left(x_{(2)} \mid x \zeta_{0}\right)\right) \Pi_{w}\right\}^{-1} .\right.
\end{aligned}
$$

From (5.22), (5.25), and (5.26), we note that $\hat{\beta}_{p}$ has the same limiting distribution of the following IV estimator:

$$
\tilde{\beta}_{p}=\left(\hat{P}^{\prime} \hat{W}\right)^{-1} \hat{P}^{\prime} \hat{Y},
$$


where

$$
\hat{P}=\left(\begin{array}{c}
t_{n}\left(x_{1} \zeta_{0}, \xi_{0}\right)\left(p_{1}-\mathrm{E}_{n}\left(p \mid x_{1} \hat{\zeta}\right)\right) \\
\vdots \\
t_{n}\left(x_{n} \zeta_{0}, \xi_{0}\right)\left(p_{n}-\mathrm{E}_{n}\left(p \mid x_{n} \hat{\zeta}\right)\right)
\end{array}\right) .
$$

This indicates that for the estimation of the semiparametric simultaneous equation model, the residuals $p_{i}-\mathrm{E}_{n}\left(p \mid x_{i} \zeta_{0}\right)$ and $p-\mathrm{E}\left(p \mid x \zeta_{0}\right)$ play the crucial role rather than the variable $p$ itself. The asymptotic covariance matrix of $\widetilde{\beta}_{p}$ (or $\hat{\beta}_{p}$ ) can be consistently estimated by $\hat{\Omega}_{p, n}=\left[\hat{P}^{\prime} \hat{W}\right]^{-1} \hat{P}^{\prime} \hat{\Sigma} \hat{P}\left[\hat{W}^{\prime} \hat{P}\right]^{-1}$. The asymptotic efficiency of $\widetilde{\beta}_{\mathrm{SG}}$ relative to $\widetilde{\beta}_{p}$ follows from the following inequality:

$$
\begin{aligned}
\Pi_{w}^{\prime} \Gamma \Pi_{w} & =\Pi_{w}^{\prime} \operatorname{plim} \frac{1}{n} \hat{X}_{2}^{\prime} \hat{\Sigma}^{-1} \hat{X}_{2} \Pi_{w} \\
& \geqslant \Pi_{w}^{\prime} \operatorname{plim} \frac{1}{n} \hat{X}_{2}^{\prime} \hat{P}\left(\hat{P}^{\prime} \hat{\Sigma} \hat{P}\right)^{-1} \hat{P}^{\prime} \hat{X}_{2} \Pi_{w} \\
& =\operatorname{plim} \frac{1}{n} \hat{W}^{\prime} \hat{P}\left(\hat{P}^{\prime} \hat{\Sigma} \hat{P}\right)^{-1} \hat{P}^{\prime} \hat{W} \\
& =\Omega_{p}^{-1} .
\end{aligned}
$$

\section{Conclusion}

In this article, we have considered the identification and estimation of the sample selection simultaneous equation model without a parametric distributional assumption. Based only on index restrictions, the identification of structural parameters from reduced form parameters requires stronger exclusion restrictions than the identification of structural equations in the classical simultaneous equation model. The identification in this semiparametric model requires the underlying structural equation to be overidentified in the classical sense. Exact identification in the classical model becomes underidentification for the semiparametric model. Estimation of the structural equation by instrumental variable methods has been considered. Some two-stage estimation procedures which generalize the estimation procedures for the parametric model and the classical two-stage least squares method are introduced. Consistency and asymptotic normality of the estimators are proved. An asymptotically efficient instrumental variable method (conditional on the same first-stage estimator of the parameters of the selection equation and the trimming of observations with low index densities) has also been derived. Some interesting features of the instrumental variable estimation in this model have been discovered. Residuals of the variables in the model which are derived from the projection of variables 
to the selection equation indices play the crucial role for asymptotic properties of the estimators. Exogenous variables in the system used in normalization for the selection equation indices behave as if they were endogenous variables in two-stage estimation procedures. The latter feature is compatible with the identification condition for the model.

In this article, we have considered only single-equation estimation methods. These methods can be generalized to the estimation of system equations by some semiparametric three-stage procedures. For the semiparametric sample selection model (without simultaneity), semiparametric efficiency bound has been derived in Chamberlain (1986). For efficient estimation, asymptotic efficient estimators might be derived from some semiparametric maximum likelihood methods. For the semiparametric sample selection model without simultaneity, Lee (1990) has considered such a procedure. ${ }^{4}$ With the reduced form equations estimated by such a method, the structural parameters may then be estimated by Amemiya's minimum distance procedure (Amemiya, 1978, 1983). One might conjecture that such a structural estimator could be an asymptotically efficient semiparametric estimator. At any rate, such estimation method is not a simple instrumental variable method.

\section{Appendix}

\section{A.1. Model assumptions}

\section{Assumption 1}

(1) The samples $s_{i}$, where $s_{i}=\left(y_{i}, z_{i}, x_{i}\right), i=1, \ldots, n$, are i.i.d. $x$ is the vector consisting of all exogenous variables in the equation system. The moments of order $3 \times r$, where $r \geqslant 2$, of $s$ exist.

(2) The parameter space $\Theta$ of $\delta$ is a compact subset of a finite-dimensional Euclidean space, and $\delta_{0}$ is in the interior of $\Theta$.

(3) $x_{(1)}$ is an $m$-dimensional vector of continuous variables.

(4) $\delta$ is a $\sqrt{n}$-consistent estimator, $\sqrt{n}\left(\hat{\delta}-\delta_{0}\right)$ is asymptotically normal, $\mathrm{N}\left(0, V_{\delta}\right)$, where $V_{\delta}$ is a positive definite matrix, and is asymptotically uncorrelated with $u_{1 i}-\mathrm{E}\left(u_{1 i} \mid x_{i} \zeta_{0}\right)$ for all $i$.

\footnotetext{
${ }^{4}$ In Lee (1990), both the density of index in the selection equation and the density of the disturbances in the outcome equations are assumed to be bounded away from zero. For such a situation, there is no need to trim the observations with lower index densities. Unfortunately, such a strong assumption rules out many cases. The author is currently investigating how to relax such an assumption. Trimming procedure introduced in this article may be valuable.
} 
(5) The matrix $\Pi_{w}$ in (4.4) has full column rank.

(6) The matrices $C$ in (4.6) and $\Gamma$ in (5.12) are nonsingular.

\section{Assumption 2}

(1) $K(v)$ on $R^{m}$ is a kernel function with a bandwidth parameter $a_{n}$ defined on a bounded support $D,{ }^{5}$ i.e., $\int_{D} K(v) \mathrm{d} v=1$, and $\lim _{n \rightarrow \infty} a_{n}=0$.

(2) $K(v)$ is twice differentiable and its second-order derivatives satisfy a Lipschitz condition of order $1 .^{6}$

(3) $K(v)$ is a higher-order kernel function with zero moments up to the order $s^{*}$, $s^{*}=m+2$, i.e.,

$$
\int_{D} v_{1}^{i_{1}} \ldots v_{m}^{i_{m}} K(v) \mathrm{d} v=0,
$$

for all $0 \leqslant i_{l}, l=1, \ldots, m$, and $1 \leqslant i_{1}+\cdots+i_{m}<s^{*}$.

(4) $\left\{a_{n}\right\}$ is chosen with a rate such that $\lim _{n \rightarrow \infty}(n / \ln n) a_{n}^{(1+6 / r)(m+2)+2}=\infty$ and $\lim _{n \rightarrow \infty} n a_{n}^{2(m+2)}=\infty$, but $\lim _{n \rightarrow \infty} n a_{n}^{4 s^{*}}=0$.

\section{Assumption 3}

(1) The density function $p(t \mid \delta)$ of $t=x \zeta$ in $R^{m}$ is positive everywhere for each $\delta \in \Theta$. It is differentiable everywhere with respect to $t$ to the order $s^{*}$, and these derivatives are continuous at $(t, \delta)$ everywhere.

(2) $\mathrm{E}(s \otimes \bar{x} \mid t, \delta) p(t \mid \theta)$, where $s=(y, z, x)$ and $\bar{x}=(1, x)$, is differentiable everywhere with respect to $t$ to the order $s^{*}+1$, and these derivatives are continuous at $(t, \delta)$ everywhere. ${ }^{7}$

(3) $\mathrm{E}\left(\|s \otimes \bar{x} \otimes \bar{x}\|^{2} \mid t, \delta\right) p(t \mid \delta)$, where $s=(1, y, z, x)$ and $\bar{x}=(1, x)$, is continuous at $(t, \delta)$ everywhere.

(4) $\mathrm{E}(s \otimes \bar{x} \otimes \bar{x} \mid t, \delta) p(t \mid \delta)$, where $s=(1, y, z)$ and $\bar{x}=(1, x)$, is twice differentiable with respect to $t$ and its second-order derivatives are continuous at $(t, \delta)$ everywhere.

\footnotetext{
${ }^{5}$ The boundedness of $D$ is inessential. Relaxing this assumption will make our analysis relatively more complicated. In practice, kernel functions with bounded support are simpler to compute.

${ }^{6} \mathrm{~A}$ function $h(x)$ is said to satisfy a Lipschitz condition of order 1 if there exists a constant $c$ such that $\left\|h\left(x_{1}\right)-h\left(x_{2}\right)\right\| \leqslant c\left\|x_{1}-x_{2}\right\|$ for all $x_{1}$ and $x_{2}$.

${ }^{7} \otimes$ denotes the Kronecker product.
} 


\section{Assumption 4}

(1) $H(v)$ on $R^{m}$ is a kernel function with a bandwidth parameter $b_{n}$ defined on a bounded support.

(2) $H(v)$ is differentiable and its derivative satisfies a Lipschitz condition of order 1 .

(3) $H(v)$ is a kernel function with zero moments up to the order $h^{*}$.

(4) $\left\{b_{n}\right\}$ is chosen such that $\lim _{n \rightarrow \infty}(n / \ln n) b_{n}^{(1+6 / r)(m+1)+1}=\infty$ and $\lim _{n \rightarrow \infty} n b_{n}^{2 m}$ $=\infty$, but $\lim _{n \rightarrow \infty} n b_{n}^{4 h^{*}}=0$.

\section{Assumption 5}

(1) $\mathrm{E}(s \otimes s \mid t, \delta) p(t \mid \theta)$, where $s=(1, y, z, x)$, of $t=x \zeta$ is differentiable everywhere to the order $h^{*}$, and these derivatives are continuous at $(t, \delta)$ everywhere.

(2) $\mathrm{E}\left(\|s \otimes s\|^{2} \mid t, \delta\right) p(t \mid \delta)$, where $s=(1, y, z, x)$, is continuous at $(t, \delta)$ everywhere.

(3) $\mathrm{E}(s \otimes s \otimes \bar{x} \mid t, \delta) p(t \mid \theta)$, where $s=(1, y, z, x)$ and $\bar{x}=(1, x)$, is differentiable everywhere with respect to $t$ and this derivative is continuous at $(t, \delta)$ everywhere.

(4) $\mathrm{E}\left(\|s \otimes s \otimes \bar{x}\|^{2} \mid t, \delta\right) p(t \mid \delta)$, where $s=(1, y, z, x)$ and $\bar{x}=(1, x)$, is continuous at $(t, \delta)$ everywhere.

As pointed out in Section 4 of the text, Assumption 1(4) will, in general, be satisfied with parametric or semiparametric estimators of discrete choice models. Assumption 1(5) is the rank identification condition, and Assumption 1(6) is for the limiting distributions of the S2LS and SG2LS estimators to be welldefined with the $\sqrt{n}$ rate of convergence.

The kernel function $K$ with a bounded support in Assumption 2 has implicitly the following properties: $\int_{D}|K(v)| \mathrm{d} v$ and $\int_{D}\|v\|^{\mathrm{s}^{*}}|K(v)| \mathrm{d} v$ are finite; $K(v)$ and its firstorder derivatives are bounded; and $K(v)$ and $\partial K(v) / \partial v$ go to zero at their boundary.

The conditions in Assumptions 3 and 5 can be justified by some basic regularity conditions on the distributions of the variables in the models. However, the above assumptions are more direct. As an illustration, let $f\left(x_{(1)} \mid x_{(2)}\right)$ be the density function of $x_{(1)}$ conditional on $x_{(2)}$. Since $t=x_{(1)}+x_{(2)} \delta$,

$$
p(t \mid \delta)=\int f\left(t-x_{(2)} \delta \mid x_{(2)}\right) \mathrm{d} v\left(x_{(2)}\right)
$$

where $v\left(x_{(2)}\right)$ is the distribution measure of $x_{(2)}$. If $f\left(x_{(1)} \mid x_{(2)}\right)$ is continuous and bounded, the bounded convergence theorem will imply that $p(t \mid \delta)$ is a continuous function. The continuity and boundedness properties in Assumptions 3 and 
5 are used to guarantee the stochastic convergence and to control asymptotic biases of nonparametric kernel estimates. See Lemmas 1 to 4 in Section A.4. Assumptions 4 and 5 are needed only for the nonparametric estimates $\omega_{n i}$ of the variance of $u_{1 i}$. For the asymptotic properties of the S2LS estimator, these two assumptions are not needed.

\section{A.2. Trimming index}

To trim the tails of the index $x \hat{\zeta}$, we can use some quantile statistics of $x \hat{\zeta}$. Without loss of generality, consider a single index. Let $0<p<\frac{1}{2}$ be a specified order of quantile. The first-stage estimate $\hat{\delta}$ in $x \hat{\xi}=x_{(1)}+x_{(2)} \hat{\delta}$ can be discretized by LeCam's device (LeCam, 1960). Let $\left\{\hat{\delta}_{n}\right\}$ be a sequence of $\sqrt{n}$-consistent estimate of $\delta_{0}$. Let $\|\delta\|=\max _{l=1, \ldots, k}\left|\delta_{l}\right|$, where $\delta=\left(\delta_{1}, \ldots, \delta_{k}\right)^{\prime}$ is the norm of $\delta$ in the $k$-dimensional Euclidean space $R^{k}$. Let $R_{n}^{k}=\{(1 / \sqrt{n})$ $\times\left(i_{1}, \ldots, i_{k}\right) \mid i_{1}, \ldots, i_{k}$ are integers $\}$ and let $\bar{\delta}_{n}$ be a point in $R_{n}^{k}$ closest to $\hat{\delta}_{n}$ under $\|\cdot\|$. The $\left\{\bar{\delta}_{n}\right\}$ is a discretized sequence of estimates of $\delta_{0}$. Let $\hat{\xi}_{n p}$ and $\hat{\zeta}_{n(1-p)}$ be respectively the $p$ th sample quantile and the $(1-p)$ th sample quantile of the observations of $x_{i} \bar{\zeta}, i-1, \ldots, n$, wherc $x_{\bar{\zeta}}=x_{(1)}+x_{(2)} \bar{\delta}_{n}$. Observations of $x_{i} \hat{\zeta}$ will be trimmed whenever their values lie outside $T_{n}$, where $T_{n}=\left[\hat{\xi}_{n p}, \hat{\xi}_{n(1-p)}\right]$.

The discretization device provides some technical simplification for asymptotic analysis. First of all, $\hat{\xi}_{n p}$ and $\hat{\xi}_{n(1-p)}$ can be shown to be $\sqrt{n}$-consistent. Since $\hat{\delta}_{n}$ is $\sqrt{n}$-consistent and $\left\|\hat{\delta}_{n}-\bar{\delta}_{n}\right\| \leqslant 1 / \sqrt{n}$,

$$
\begin{aligned}
\sqrt{n}\left(\bar{\delta}_{n}-\delta_{0}\right) & =\sqrt{n}\left(\bar{\delta}_{n}-\hat{\delta}_{n}\right)+\sqrt{n}\left(\hat{\delta}_{n}-\delta_{0}\right) \\
& =\mathrm{O}_{p}(1),
\end{aligned}
$$

i.e., $\bar{\delta}_{n}$ is also $\sqrt{n}$-consistent. For any $\delta$, the samples $x_{i} \zeta$ are i.i.d. Let $\xi_{p}(\delta)$ be the $p$ th quantile of $x \zeta$ and $\xi_{n p}(\delta)$ be the corresponding $p$ th sample quantile of $x_{i} \zeta$, $i=1, \ldots, n$. From Theorem 2.2.1 in Serfling (1980), we know that for any $\varepsilon>0$,

$$
P\left(\left|\xi_{n p}(\delta)-\xi_{p}(\delta)\right|>\varepsilon\right) \leqslant 2 \exp \left(-n \delta_{\varepsilon}^{2}\right), \quad n \geqslant 1,
$$

where $\delta_{\varepsilon}=\min \left[F_{\delta}\left(\xi_{p}(\delta)+\varepsilon\right)-p, p-F_{\delta}\left(\xi_{p}(\delta)-\varepsilon\right)\right]$ and $F_{\delta}$ denotes the distribution of $x \zeta{ }^{8}$ Under Assumption 3(1), the density function $p(t \mid \delta)$ of $x \zeta$ is positive everywhere, and hence is bounded away from zero on any compact neighborhood $\mathcal{N}\left(\xi_{p}\left(\delta_{0}\right)\right) \times \mathcal{N}\left(\delta_{0}\right)$ of $\left(\xi_{p}\left(\delta_{0}\right), \delta_{0}\right)$. With (A.2.1), we can show that for any sequence $\left\{\delta_{n}\right\}$ such that $\lim _{n \rightarrow \infty} \delta_{n}=\delta_{0}$,

$$
\sqrt{n}\left|\xi_{n p}\left(\delta_{n}\right)-\xi_{p}\left(\delta_{n}\right)\right|=\mathrm{O}_{p}(1)
$$

\footnotetext{
${ }^{8}$ This exponential bound for the sample quantile follows from Hoeffding's inequality.
} 
Let $\varepsilon_{n}=c / \sqrt{n}$, where $c$ is an arbitrary constant. By the mean value theorem, $F_{\delta_{n}}\left(\xi_{p}\left(\delta_{n}\right)+\varepsilon_{n}\right)-p=p\left(\xi_{p}\left(\delta_{n}\right)+\lambda_{n} \varepsilon_{n} \mid \delta_{n}\right) \varepsilon_{n}$ for some $\lambda_{n}, 0 \leqslant \lambda_{n} \leqslant 1$. By the continuity of $\xi_{p}(\delta)$ at $\delta=\delta_{0},\left(\xi_{p}\left(\delta_{n}\right)+\lambda_{n} \varepsilon_{n}, \delta_{n}\right) \in \mathscr{N}\left(\xi_{p}\left(\delta_{0}\right)\right) \times \mathscr{N}\left(\delta_{0}\right)$ for sufficiently large $n$. It follows that for large $n, F_{\delta_{n}}\left(\xi_{p}\left(\delta_{n}\right)+\varepsilon_{n}\right)-p \geqslant b \varepsilon_{n}$, where $b=$ $\inf _{(t, \delta) \in \mathcal{N}\left(\xi_{p}\left(\delta_{0}\right)\right) \times \mathcal{N}\left(\delta_{0}\right)} p(t \mid \delta)>0$. Similarly, $p-F_{\delta_{n}}\left(\xi_{p}\left(\delta_{n}\right)-\varepsilon_{n}\right) \geqslant b \varepsilon_{n}$. Therefore, for large $n, \exp \left(-n \delta_{\varepsilon_{n}}^{2}\right) \leqslant \exp \left(-n b^{2} \varepsilon_{n}^{2}\right)=\exp \left(-b^{2} c^{2}\right)$, and

$$
P\left(\sqrt{n}\left|\xi_{n p}\left(\delta_{n}\right)-\xi_{p}\left(\delta_{n}\right)\right|>c\right) \leqslant 2 \exp \left(-b^{2} c^{2}\right) .
$$

Since $c$ is arbitrary, it follows that $\sqrt{n}\left|\xi_{n p}\left(\delta_{n}\right)-\xi_{p}\left(\delta_{n}\right)\right|=\mathrm{O}_{p}(1)$ for any sequence $\left\{\delta_{n}\right\}$ which converges to $\delta_{0}$. For any finite constant $M>0$, define $\Delta_{M, n}=\left\{\delta \mid\left\|\delta-\delta_{0}\right\|<M / \sqrt{n}\right.$ and $\left.\delta \in R_{n}^{k}\right\} . \Delta_{M, n}$ has the interesting property that its cardinality is finite and bounded, say by $\bar{M}$, independently of $n$. This is so since the cardinality of $\left\{\left(i_{1}, \ldots, i_{k}\right) \mid i_{1}, \ldots, i_{k}\right.$ are integers and $\left|i_{l}-\sqrt{n} \delta_{0, l}\right|<M$, $l=1, \ldots, k\}$ is finite and bounded, independently of $n$. It is obvious that for any sequence $\left\{\delta_{n}\right\}$ with $\delta_{n} \in \Delta_{M, n}$, it converges to $\delta_{0}$. The finiteness of $\Delta_{M, n}$ and (A.2.3) imply that

$$
P\left(\sup _{\delta_{n} \in \boldsymbol{A}_{M, n}} \sqrt{n}\left|\xi_{n p}\left(\delta_{n}\right)-\xi_{p}\left(\delta_{n}\right)\right|>c\right) \leqslant 2 \bar{M} \exp \left(-b^{2} c^{2}\right),
$$

for large $n$, and therefore

$$
\sup _{\delta_{n} \in \Delta_{M, n}} \sqrt{n}\left|\xi_{n p}\left(\delta_{n}\right)-\xi_{p}\left(\delta_{n}\right)\right|=\mathrm{O}_{P}(1) .
$$

Since $\bar{\delta}_{n}$ is in $R_{n}^{k}$ and is a $\sqrt{n}$-consistent estimate of $\delta_{0}, \bar{\delta}_{n}$ will lie in $\Delta_{M, n}$ with probability close to one for large $n$. Hence

$$
\sqrt{n}\left|\xi_{n p}\left(\bar{\delta}_{n}\right)-\xi_{p}\left(\bar{\delta}_{n}\right)\right|=\mathrm{O}_{P}(1)
$$

By the mean value theorem, $\xi_{p}\left(\bar{\delta}_{n}\right)=\xi_{p}\left(\delta_{0}\right)+\left(\partial \xi_{p}\left(\delta_{n}^{*}\right) / \partial \delta^{\prime}\right)\left(\bar{\delta}_{n}-\delta_{0}\right)$. Since $p(t \mid \delta)$ is continuous, $\partial \xi_{p}(\delta) / \partial \delta$ is continuous at $\delta_{0}$, and $\sqrt{n}\left(\bar{\delta}_{n}-\delta_{0}\right)=\mathrm{O}_{p}(1)$, it follows that

$$
\begin{aligned}
\sqrt{n}\left|\xi_{n p}\left(\bar{\delta}_{n}\right)-\xi_{p}\left(\delta_{0}\right)\right| & \leqslant \sqrt{n}\left|\xi_{n p}\left(\bar{\delta}_{n}\right)-\xi_{p}\left(\bar{\delta}_{n}\right)\right|+\left\|\frac{\partial \xi_{p}\left(\delta_{n}^{*}\right)}{\partial \delta^{\prime}}\right\| \sqrt{n}\left|\bar{\delta}_{n}-\delta_{0}\right| \\
& =\mathrm{O}_{p}(1),
\end{aligned}
$$

i.e., $\hat{\xi}_{n p}$ is a $\sqrt{n}$-consistent estimate of $\xi_{p}\left(\delta_{0}\right)$. Similarly, $\hat{\xi}_{n(1-p)}$ is a $\sqrt{n}$-consistent estimate of $\xi_{(1-p)}\left(\delta_{0}\right)$.

The LeCam discretization device is also useful in the following way. Denote $\hat{\xi}_{n}=\left(\hat{\xi}_{n p}, \hat{\xi}_{n(1-p)}\right)$ and $\xi_{0}=\left(\xi_{p}\left(\delta_{0}\right), \xi_{(1-p)}\left(\delta_{0}\right)\right)$. For any statistic $S_{n}\left(\hat{\xi}_{n}\right)$ constructed from the sample and $\hat{\xi}_{n}$, if we want to show that $S_{n}\left(\hat{\xi}_{n}\right)$ converges to 0 in 
probability, it is sufficient to show that $S_{n}\left(\xi_{0}+(1 / \sqrt{n}) h_{n}\right)$, where $\left\{h_{n}\right\}$ is any nonstochastic bounded sequence, converges to 0 in probability. Effectively, one can replace the stochastic sequence $\left\{\hat{\xi}_{n}\right\}$ by nonstochastic sequence $\left\{\xi_{0}+(1 / \sqrt{n}) h_{n}\right\}$ in the proof of convergence in probability (see LeCam, 1960; Manski, 1984).

\section{A.3. Smooth trimming}

The trimming can be smoothed by down weighting the observations $x_{i} \hat{\zeta}$ near the sample quantiles $\hat{\xi}_{n p}$ and $\hat{\xi}_{n(1-p)}$. Let $h_{n}$ be a sequence of positive numbers which converge to zero with a rate such that $\lim _{n \rightarrow \infty}(n / \ln n) h_{n}^{(1+4 / r)}=\infty$, and let $q(\cdot)$ be a continuous density function on [0 1 l such that $q(0)=q(1)=0 .^{9}$ Denote $\xi_{n}=\left(\xi_{n p}, \xi_{n(1-p)}\right)$. Define the following smooth trimming function $t_{n}$ of $x \zeta$ :

$$
t_{n}\left(x \zeta, \xi_{n}\right)= \begin{cases}0 & \text { if } x \zeta<\zeta_{n p}, \\ \int_{0}^{x \zeta-\xi_{n p}} \frac{\xi_{n}}{h_{n}} q(w) \mathrm{d} w & \text { if } \xi_{n p} \leqslant x \zeta \leqslant \xi_{n p}+h_{n}, \\ 1 & \text { if } \xi_{n p}+h_{n}<x \zeta<\xi_{n(1-p)}-h_{n}, \\ 1-\int_{0}^{\frac{x \zeta-\xi_{n(1-p)}}{h_{n}}+1} q(w) \mathrm{d} w & \text { if } \quad \xi_{n(1-p)}-h_{n} \leqslant x \zeta \leqslant \xi_{n(1-p)}, \\ 0 & \text { if } \quad \xi_{n(1-p)} \leqslant x \zeta .\end{cases}
$$

This function is continuously differentiable in $\delta, \xi_{n p}$, and $\xi_{n(1-p)}$, with

$$
\begin{aligned}
& \frac{\partial t_{n}\left(x \zeta, \xi_{n}\right)}{\partial \delta^{\prime}}= \begin{cases}0 & \text { if } x \zeta<\xi_{n p}, \\
\frac{1}{h_{n}} q\left(\frac{x \zeta-\xi_{n p}}{h_{n}}\right) x_{(2)} & \text { if } \xi_{n p} \leqslant x \zeta \leqslant \xi_{n p}+h_{n}, \\
0 & \text { if } \xi_{n p}+h_{n}<x \zeta<\xi_{n(1-p)}-h_{n}, \\
-\frac{1}{h_{n}} q\left(\frac{x \zeta-\xi_{n(1-p)}}{h_{n}}+1\right) x_{(2)} & \text { if } \xi_{n(1-p)}-h_{n} \leqslant x \zeta \leqslant \xi_{n(1-p),} \\
0 & \text { if } \xi_{n(1-p)} \leqslant x \zeta,\end{cases} \\
& \frac{\partial t_{n}\left(x \zeta, \xi_{n}\right)}{\partial \xi_{n p}}=\left\{\begin{array}{lll}
0 & \text { if } x \zeta<\xi_{n p}, \\
-\frac{1}{h_{n}} q\left(\frac{x \zeta-\xi_{n p}}{h_{n}}\right) & \text { if } \xi_{n p} \leqslant x \zeta \leqslant \xi_{n p}+h_{n}, \\
0 & \text { if } \xi_{n p}+h_{n}<x \zeta,
\end{array}\right.
\end{aligned}
$$

\footnotetext{
${ }^{9}$ The rate of convergence of $h_{n}$ is designed to justify our asymptotic analysis (see Proposition 2 of Section A.4).
} 
and

$$
\frac{\partial t_{n}\left(x \zeta, \xi_{n}\right)}{\partial \xi_{n(1-p)}}=\left\{\begin{array}{lll}
0 & \text { if } \quad x \zeta<\xi_{n(1-p)}-h_{n}, \\
\frac{1}{h_{n}} q\left(\frac{\left.x \zeta-\xi_{n(1-p)}+1\right)}{h_{n}}\right) & \text { if } \quad \xi_{n(1-p)}-h_{n} \leqslant x \zeta \leqslant \xi_{n(1-p)}, \\
0 & \text { if } \quad \xi_{n(1-p)}<x \zeta .
\end{array}\right.
$$

As $n$ tends to infinity, $t_{n}\left(x \hat{\xi}, \hat{\xi}_{n}\right)$ will converge in probability to the indicator function $I_{T}\left(x \zeta_{0}\right)$, where $T=\left[\xi_{p}\left(\delta_{0}\right), \xi_{(1-p)}\left(\delta_{0}\right)\right]$.

The above trimming procedure can be generalized to models with multiple indices. For each index, it can be trimmed with the univariate function $t_{n}\left(x \hat{\zeta}, \hat{\xi}_{n}\right)$ above. The smooth trimming function can then be the product of all such univariate trimming functions.

A.4. Some useful asymptotic properties of nonparametric estimators of unknown functions

The following Lemma 1 provides a uniform law of large numbers for functions with a bandwidth sequence of parameters. Its proof relies on Hoeffding inequality (or Bernstein inequality) and can be found in Ichimura and Lee (1991). Lemmas 2, 3, and 4 provide results on the asymptotic biases of functions involving a kernel function and its first- and second-order derivatives. The proofs of Lemmas 2, 3, and 4 are also abstracted from Ichimura and Lee (1991).

Lemma 1. Let $g\left(z, a_{n}, \beta\right)$ be a measurable function which can be represented in the form

$$
g\left(z, a_{n}, \beta\right)=\frac{1}{a_{n}^{d}} c(z, \beta) h\left[z, \beta, \frac{s(z, \beta)}{a_{n}}\right],
$$

where $a_{n}=\mathrm{O}\left(1 / n^{p}\right), p>0, d>0, \beta \in B$, and $s(z, \beta)$ is a finite-dimensional vector value function. Let $\left\{z_{i}\right\}$ be a sequence of i.i.d. random vectors. Suppose that the following conditions are satisfied:

(i) $B$ is a compact subset of a finite-dimensional Euclidean space.

(ii) $c(z, \beta)$ is bounded by an l-order polynomial of $z$ uniformly in $\beta$, where $l \geqslant 0$.

(iii) The first $l \times r$ moments of $z$ exist, where $r \geqslant 2$.

(iv) $h(\cdot)$ is a bounded function.

(v) $\mathrm{E}\left[c^{2}(z, \beta) h^{2}\left(z, \beta, s(z, \beta) / a_{n}\right)\right]=\mathrm{O}\left(a_{n}^{\bar{d}}\right)$ uniformly in $\beta \in B$, where $\bar{d} \leqslant d$.

(vi) $h(z, \beta, s)$ satisfies the Lipschizian condition of order $I$ with respect to $\beta$ and $s$ uniformly in $z ; s(z, \beta)$ satisfies the Lipschizian condition of order $I$ with respect to $\beta$ uniformly in $z$. 
If $\lim _{n \rightarrow \infty}(n / \ln n) a_{n}^{2(1+l / r) d-\bar{d}}=\infty$, then $(1 / n) \sum_{i=1}^{n}\left[g\left(z_{i}, a_{n}, \beta\right)-\mathrm{E}\left(g\left(z_{i}, a_{n}, \beta\right)\right)\right]$ converges in probability to zero uniformly in $\beta \in B$.

Furthermore, in addition to the above conditions, if:

(vii) $\mathbf{E}\left(g\left(z, a_{n}, \beta\right)\right)$ converges to a limit function $g^{*}(\beta)$ uniformly in $\beta \in B$, then $(1 / n) \sum_{i=1}^{n} g\left(z_{i}, a_{n}, \beta\right)$ converges in probability to $g^{*}(\beta)$ uniformly in $\beta \in B$.

Lemma 2. Let $K(v)$ be a function on $R^{m}$ with a bounded support $D$ such that $\int_{D}|K(v)| \mathrm{d} v<\infty . t(z, \theta)$ is a continuous $m$-dimensional random vector. $z_{i}$ is a point in the support of $z$. Suppose that $\mathrm{E}\left(c\left(z, z_{i}, \theta\right) \mid t(z, \theta)=t, z_{i}\right) g(t \mid \theta)$, where $g(t \mid \theta)$ is the density function of $t(z, \theta)$, is uniformly continuous at $t$ on $R^{m}$ uniformly in $\left(\theta, z_{i}\right)$. Then

$$
\begin{aligned}
& \sup _{z_{i}, \theta} \mid \mathrm{E}\left[c\left(z, z_{i}, \theta\right) \frac{1}{a_{n}^{m}} K\left(\frac{t\left(z_{i}, \theta\right)-t(z, \theta)}{a_{n}}\right) \mid z_{i}\right] \\
& -\mathrm{E}\left[c\left(z, z_{i}, \theta\right) \mid t(z, \theta)=t\left(z_{i}, \theta\right), z_{i}\right] g\left(t\left(z_{i}, \theta\right) \mid \theta\right) \mid \rightarrow 0 .
\end{aligned}
$$

Furthermore, if $K(v)$ has zero moments up to the order $s^{*}$; i.e., $\int_{D} v_{1}^{i_{1}} \cdots v_{m}^{i_{m}}$ $\times K(v) \mathrm{d} v=0$, for all $i_{j} \geqslant 0$, where $j=1, \ldots, m, i_{1}+\cdots+i_{m}<s^{*}$, and $\int_{D}\|v\|^{s^{*}}|K(v)| \mathrm{d} v<\infty ;$ and $\mathrm{E}\left(c\left(z, z_{i}, \theta\right) \mid t(z, \theta)=t, z_{i}\right) g(t \mid \theta)$ is differentiable with $t$ on $R^{m}$ to the order $s^{*}$ and the $s^{*}$-order derivatives are uniformly bounded, then

$$
\begin{aligned}
& \sup _{z_{i}, \theta} \mid \mathrm{E}\left[c\left(z, z_{i}, \theta\right) \frac{1}{a_{n}^{m}} K\left(\frac{t\left(z_{i}, \theta\right)-t(z, \theta)}{a_{n}}\right) \mid z_{i}\right] \\
& -\mathrm{E}\left[c\left(z, z_{i}, \theta\right) \mid t(z, \theta)=t\left(z_{i}, \theta\right), z_{i}\right] g\left(t\left(z_{i}, \theta\right) \mid \theta\right) \mid=\mathrm{O}\left(a_{n}^{s^{*}}\right) .
\end{aligned}
$$

Lemma 3. Let $K(v)$ be a function on $R^{m}$ with a bounded support $D$ such that $K(v)$ goes to zero at the boundary of $D$ and its gradient $\partial K(v) / \partial v$ is bounded. Suppose that $(\partial / \partial t)\left[\mathrm{E}\left(c\left(z, z_{i}, \theta\right) \mid t(z, \theta)=t, z_{i}\right) g(t \mid \theta)\right]$, where $g(t \mid \theta)$ is the density function $t(z, \theta)$, are uniformly continuous at $t$ uniformly in $\left(z_{i}, \theta\right)$. Then

$$
\begin{aligned}
& \lim _{n \rightarrow \infty} \sup _{z_{i}, \theta} \mid \mathrm{E}\left[c\left(z, z_{i}, \theta\right) \frac{1}{a_{n}^{m+1}} \frac{\partial K\left(\frac{t\left(z_{i}, \theta\right)-t(z, \theta)}{a_{n}}\right)}{\partial v} \mid z_{i}\right] \\
& -\frac{\partial}{\partial t}\left\{\mathrm{E}\left[c\left(z, z_{i}, \theta\right) \mid t(z, \theta)=t\left(z_{i}, \theta\right), z_{i}\right] g\left(t\left(z_{i}, \theta\right) \mid \theta\right)\right\} \mid=0 .
\end{aligned}
$$


Furthermore, if $K(v)$ has zero moments up to the order $s^{*}$, $\mathrm{E}\left(c\left(z, z_{i}, \theta\right) \mid t(z, \theta)=t, z_{i}\right) g(t \mid \theta)$ is differentiable at $t$ everywhere to the order $s^{*}+1$, and these derivatives are uniformly bounded, then

$$
\begin{aligned}
& \sup _{z_{i}, \theta} \mid \mathrm{E}\left[c\left(z, z_{i}, \theta\right) \frac{1}{a_{n}^{m+1}} \frac{\partial K\left(\frac{t\left(z_{i}, \theta\right)-t(z, \theta)}{a_{n}}\right)}{\partial v} \mid z_{i}\right] \\
& -\frac{\partial}{\partial t}\left\{\mathrm{E}\left[c\left(z, z_{i}, \theta\right) \mid t(z, \theta)=t\left(z_{i}, \theta\right), z_{i}\right] g\left(t\left(z_{i}, \theta\right) \mid \theta\right)\right\} \mid=\mathrm{O}\left(a_{n}^{s^{*}}\right) .
\end{aligned}
$$

Lemma 4. Let $K(v)$ be a twice differentiable function on $R^{m}$ with a bounded support $D$ such that $K(v)$ and its gradient $\partial K(v) / \partial v$ go to zero at the boundary of $D$, and the gradient $\partial K(v) / \partial v$ and its Hessian matrix $\partial^{2} K(v) / \partial v \partial v^{\prime}$ are bounded. Suppose that $\left(\partial^{2} / \partial t \partial t^{\prime}\right)\left[\mathrm{E}\left(c\left(z, z_{i}, \theta\right) \mid t(z, \theta)=t, z_{i}\right) g(t \mid \theta)\right]$ are uniformly continuous at $t$ everywhere uniformly in $\left(z_{i}, \theta\right)$. Then

$$
\begin{gathered}
\lim _{n \rightarrow \infty} \sup _{z_{i}, \theta} \mid \mathrm{E}\left[c\left(z, z_{i}, \theta\right) \frac{1}{a_{n}^{m+2}} \frac{\partial^{2} K\left(\frac{t\left(z_{i}, \theta\right)-t(z, \theta)}{a_{n}}\right)}{\partial v \partial v^{\prime}} \mid z_{i}\right] \\
-\frac{\partial^{2}}{\partial v \partial v^{\prime}}\left\{\mathrm{E}\left[c\left(z, z_{i}, \theta\right) \mid t(z, \theta)=t\left(z_{i}, \theta\right), z_{i}\right] g\left(t\left(z_{i}, \theta\right) \mid \theta\right)\right\} \mid=0 .
\end{gathered}
$$

Let $x \zeta$ be an $m$-dimensional vector of indices and $K$ be an $m$-dimensional kernel function. Denote

$$
A_{n}\left(s \mid x_{i} \zeta\right)=\frac{1}{(n-1) a_{n}^{m}} \sum_{j \neq i}^{n} s_{j} K\left(\frac{x_{i} \zeta-x_{j} \zeta}{a_{n}}\right),
$$

where $s=1, y, z$, or $x$. Under Assumption 3(3), the variance of $A_{n}\left(w \mid x_{i} \zeta\right)$ has the familiar order $\mathrm{O}\left(1 / n a_{n}^{m}\right)$ uniformly on $C \times \Theta$, where $C$ is any compact subset of $x \zeta$ in $R^{m}$; i.e.,

$$
\sup _{C \times \Theta} \operatorname{var}\left(A_{n}\left(s \mid x_{i} \zeta\right) \mid x_{i}\right)=\mathrm{O}\left(\frac{1}{n a_{n}^{m}}\right) .
$$


Assumption 2 implies that $K(\cdot)$ is bounded. It follows from Lemma 1 that if $\lim _{n \rightarrow \infty}(n / \ln n) a_{n}^{(1+2 / r) m}=\infty$,

$$
\sup _{\left(x_{i} \zeta, \theta\right) \in C \times \Theta}\left|A_{n}\left(s \mid x_{i} \zeta\right)-\mathrm{E}\left[A_{n}\left(s \mid x_{i} \zeta\right) \mid x_{i}\right]\right| \stackrel{p}{\rightarrow} 0 .
$$

Under Assumptions 3(2) and 3(3), Lemma 2 guarantees that

$$
\sup _{C \times \Theta}\left|\mathrm{E}\left[A_{n}\left(s \mid x_{i} \zeta\right) \mid x_{i}\right]-A\left(s \mid x_{i} \zeta, \delta\right)\right|=\mathrm{O}\left(a_{n}^{s^{*}}\right),
$$

where

$$
A\left(s \mid x_{i} \zeta, \delta\right)=\left.[\mathrm{E}(s \mid x \zeta) p(x \zeta \mid \delta)]\right|_{x \zeta=x_{i} \zeta},
$$

i.e., $A\left(s \mid x_{i} \zeta, \delta\right)$ is the product of $p\left(x_{i} \zeta \mid \delta\right)$ and the conditional expectation of $s$ conditional on $x \zeta$ evaluated at the point $x_{i} \zeta$. To simplify notation in subsequent presentation, we adopt the convention that $\mathrm{E}\left(s \mid x \zeta=x_{i} \zeta\right)$ denotes the conditional expectation $\mathrm{E}(s \mid x \zeta)$ evaluated at the point $x_{i} \zeta$, i.e., $\mathrm{E}\left(s \mid x \zeta=x_{i} \zeta\right)=\left.\mathrm{E}(s \mid x \zeta)\right|_{x_{\zeta}=x_{i} \zeta}$, for any random variable $s$. Since with probability close to one, $T_{n}$ will be contained in a compact subset of $R^{m}$ for large $n$, $A_{n}\left(s \mid x_{i} \zeta\right)$ converges in probability to $A\left(s \mid x_{i} \zeta, \delta\right)$ on $T_{n} \times \Theta$. Since $p(x \zeta \mid \delta)$ is continuous and is positive everywhere, it is bounded away from zero on $T_{n} \times \Theta$. Hence the uniform convergence of $A_{n}\left(1 \mid x_{i} \zeta\right)$ implies that $\inf _{T_{n \times \Theta}} A_{n}\left(1 \mid x_{i} \zeta\right)$ is bounded away from zero in probability. ${ }^{10} \mathrm{E}_{n}\left(s \mid x_{i} \zeta\right)$ as a ratio of $A_{n}\left(s \mid x_{i} \zeta\right)$ over $A_{n}\left(1 \mid x_{i} \zeta\right)$ will converge in probability to $\mathrm{E}\left(s \mid x \zeta=x_{i} \zeta\right)$ uniformly on $T_{n} \times \Theta$.

The first-order derivative of $A_{n}\left(s \mid x_{i} \zeta\right)$ is

$$
\frac{\partial A_{n}\left(s \mid x_{i} \zeta\right)}{\partial \delta_{k}}=\frac{1}{(n-1) a_{n}^{m+1}} \sum_{j \neq i}^{n} s_{j}\left(x_{i}-x_{j}\right) \frac{\partial \zeta}{\partial \delta_{k}} \frac{\partial K\left(\frac{x_{i} \zeta-x_{j} \zeta}{a_{n}}\right)}{\partial v} .
$$

Under Assumption 3(3), the variance of $\partial A_{n}\left(s \mid x_{i} \zeta\right) / \partial \delta$ has the familiar order $\mathrm{O}\left(1 / n a_{n}^{m+2}\right)$ uniformly in $\left(x_{i} \zeta, \delta\right) \in C \times \Theta$. Assumption $3(2)$ justifies the conditions in Lemma 3 and hence

$$
\sup _{c \times \Theta}\left|\mathrm{E}\left[\frac{\partial A_{n}\left(s \mid x_{i} \zeta\right)}{\partial \delta_{k}} \mid x_{i} \zeta\right]-\frac{\partial A\left(s \mid x_{i} \zeta, \delta\right)}{\partial \delta_{k}}\right|=\mathrm{O}\left(a_{n}^{s^{*}}\right),
$$

where

$$
\begin{aligned}
\frac{\partial A\left(s \mid x_{i} \zeta, \delta\right)}{\partial \delta_{k}}= & \operatorname{tr}\left\{\frac{\partial \mathrm{E}\left(s\left(x_{i}-x\right) \mid x \zeta=x_{i} \zeta, x_{i}\right)}{\partial t} \frac{\partial \zeta}{\partial \delta_{k}}\right\} p\left(x_{i} \zeta \mid \delta\right) \\
& +\mathrm{E}\left(s\left(x_{i}-x\right) \mid x \zeta=x_{i} \zeta, x_{i}\right) \frac{\partial \zeta}{\partial \delta_{k}} \nabla p\left(x_{i} \zeta \mid \delta\right)
\end{aligned}
$$

\footnotetext{
${ }^{10}$ The trimming of indices is designed mainly for this purpose. Otherwise, trimming would not be needed.
} 
where $\nabla p(x \zeta \mid \delta)$ denotes the first-order derivative of $p(x \zeta \mid \delta)$ with respect to $x \zeta$ (see Ichimura and Lee, 1991). Lemmas 1 and 3 imply that if $\lim _{n \rightarrow \infty}(n / \ln n) a_{n}^{(1+4 / r)(m+1)+1}=\infty$,

$$
\sup _{T_{n} \times \Theta}\left|\frac{\partial A_{n}\left(s \mid x_{i} \zeta\right)}{\partial \delta}-\frac{\partial A\left(s \mid x_{i} \zeta, \delta\right)}{\partial \delta}\right| \stackrel{p}{\rightarrow} 0 .
$$

Similarly, under Assumptions 3(3) and 3(4), Lemmas 1 and 4 imply that if $\lim _{n \rightarrow \infty}(n / \ln n) a_{n}^{(1+6 / r)(m+2)+2}=\infty$,

$$
\sup _{T_{n} \times \Theta}\left|\frac{\partial^{2} A_{n}\left(s \mid x_{i} \zeta\right)}{\partial \delta \partial \delta^{\prime}}-\frac{\partial^{2} A\left(s \mid x_{i} \zeta, \delta\right)}{\partial \delta \partial \delta^{\prime}}\right| \stackrel{p}{\rightarrow} 0 .
$$

Since $\mathrm{E}_{n}\left(s \mid x_{i} \zeta\right)=A_{n}\left(s \mid x_{i} \zeta\right) / A_{n}\left(1 \mid x_{i} \zeta\right)$,

$$
\frac{\partial \mathrm{E}_{n}\left(s \mid x_{i} \zeta\right)}{\partial \delta}=\left(\frac{\partial A_{n}\left(s \mid x_{i} \zeta\right)}{\partial \delta}-\mathrm{E}_{n}\left(s \mid x_{i} \zeta\right) \frac{\partial A_{n}\left(1 \mid x_{i} \zeta\right)}{\partial \delta}\right) / A_{n}\left(1 \mid x_{i} \zeta\right)
$$

converges in probability to $\partial \mathrm{E}\left(s \mid x \zeta=x_{i} \zeta\right) / \partial \delta$, where

$$
\frac{\partial \mathrm{E}(s \mid x \zeta)}{\partial \delta}=\left(\frac{\partial A(s \mid x \zeta, \delta)}{\partial \delta}-\mathrm{E}(s \mid x \zeta) \frac{\partial A(1 \mid x \zeta, \delta)}{\partial \delta}\right) / A(1 \mid x \zeta, \delta)
$$

from (A.4.3), (A.4.4), and (A.4.9). Since $\mathrm{E}\left(u_{1} \mid x\right)=\mathrm{E}\left(u_{1} \mid x \zeta_{0}\right)$, as shown in Ichimura and Lee (1991, Lemma 4), we have explicitly the following expression:

$$
\left.\frac{\partial \mathrm{E}\left(u_{1} \mid x \zeta\right)}{\partial \delta_{k}}\right|_{\delta=\delta_{0}}=\left(x-\mathrm{E}\left(x \mid x \zeta_{0}\right)\right) \frac{\partial \zeta\left(\delta_{0}\right)}{\partial \delta_{k}} \nabla \mathrm{E}\left(u_{1} \mid x \zeta_{0}\right),
$$

where $\nabla \mathrm{E}\left(u_{1} \mid x \zeta_{0}\right)$ is the first-order derivative of $\mathrm{E}\left(u_{1} \mid x \zeta_{0}\right)$ with respect to $x \zeta_{0}$.

The following propositions and lemmas will be used repeatedly for our asymptotic analysis in Section A.5. They are summarized here because they are of interest on their own and provide convenient reference.

Proposition 1. Let $\mathscr{N}\left(\xi_{0}\right)$ be a compact neighborhood of $\xi_{0}=\left(\xi_{p}\left(\delta_{0}\right), \xi_{(1-p)}\left(\delta_{0}\right)\right)$ such that for any $\xi=\left(\xi_{p}, \xi_{(1-p)}\right) \in \mathscr{N}\left(\xi_{0}\right),\left[\xi_{p}, \xi_{(1-p)}\right] \subseteq \bar{T}$ where $\bar{T}=$ $\left[\xi_{p}\left(\delta_{0}\right)-\Delta, \xi_{(1-p)}\left(\delta_{0}\right)+\Delta\right]$ for some $\Delta>0$ (a constant not depended on $\left.\xi\right)$. Suppose that:

(1) $t_{n}\left(x_{i} \zeta, \xi\right)$ is a bounded smooth trimming function defined in Section A.3, which vanishes at any $x_{i} \zeta$ outside the interval $\left[\xi_{p}, \xi_{(1-p)}\right]$.

(2) $A_{n}\left(s \mid x_{i} \zeta\right) \stackrel{p}{\rightarrow} A\left(s \mid x_{i} \zeta, \delta\right)$ uniformly in $\left(x_{i} \zeta, \delta\right) \in \bar{T} \times \Theta$, where $\Theta$ is a compact neighborhood of $\delta_{0}$, and $A(s \mid x \zeta, \delta)$ is continuous in $\delta$ and $x \zeta$.

(3) $f(d)$ is uniformly continuous and bounded on $A_{\Delta}$, where $A_{\Delta}=$ $\{d \mid d=A(s \mid x \zeta, \delta),(x \zeta, \delta) \in \bar{T} \times \Theta\}$. 
(4) a(s) is bounded by a square integrable polynomial of finite order.

Then

$$
\begin{aligned}
& \operatorname{plim}_{n \rightarrow \infty} \sup _{\Theta \times \mathcal{N}\left(\xi_{0}\right)} \mid \frac{1}{n} \sum_{i=1}^{n} t_{n}\left(x_{i} \zeta, \xi\right) a\left(s_{i}\right) f\left(A_{n}\left(s \mid x_{i} \zeta\right)\right) \\
& -\mathrm{E}\left\{t_{n}(x \zeta, \xi) a(s) f(A(s \mid x \zeta, \delta))\right\} \mid=0 .
\end{aligned}
$$

In addition to the above assumptions, if $A(s \mid x \zeta, \delta)$ is continuous in $\delta$ and $x \zeta$ a.e., $\hat{\delta}$ is a consistent estimate of $\delta_{0}$, and $\hat{\xi}_{n}$ is a consistent estimate of $\xi_{0}$, then

$$
\operatorname{plim}_{n \rightarrow \infty} \frac{1}{n} \sum_{i=1}^{n} t_{n}\left(x_{i} \hat{\zeta}, \hat{\zeta}_{n}\right) a\left(s_{i}\right) f\left(A_{n}\left(s \mid x_{i} \hat{\zeta}\right)\right)=\mathrm{E}\left\{I_{T}\left(x_{\zeta_{0}}\right) a(s) f\left(A\left(s \mid x \zeta_{0}, \delta_{0}\right)\right)\right\},
$$

where $T=\left[\xi_{p}\left(\delta_{0}\right), \xi_{(1-p)}\left(\delta_{0}\right)\right]$.

Proof.

$$
\begin{aligned}
& \sup _{\Theta \times \mathcal{N}\left(\zeta_{0}\right)}\left|\frac{1}{n} \sum_{i=1}^{n} t_{n}\left(x_{i} \zeta, \xi\right) a\left(s_{i}\right) f\left(A_{n}\left(s \mid x_{i} \zeta\right)\right)-\frac{1}{n} \sum_{i=1}^{n} t_{n}\left(x_{i} \zeta, \xi\right) a\left(s_{i}\right) f\left(A\left(s \mid x_{i} \zeta, \delta\right)\right)\right| \\
& \leqslant \frac{1}{n} \sum_{i-1}^{n}\left|a\left(s_{i}\right)\right| \sup _{\left(x_{i} \zeta, \delta\right) \in \bar{T} \times \Theta}\left|f\left(A_{n}\left(s \mid x_{i} \zeta\right)\right)-f\left(A\left(s \mid x_{i} \zeta, \delta\right)\right)\right| \\
& \stackrel{p}{\rightarrow} 0
\end{aligned}
$$

because $t_{n}$ is bounded by one, $f\left(A_{n}\left(s \mid x_{i} \zeta\right)\right)$ converges in probability to $f\left(A\left(s \mid x_{i} \zeta, \delta\right)\right)$ uniformly on $\bar{T} \times \Theta$, and $(1 / n) \sum_{i=1}^{n}\left|a\left(s_{i}\right)\right|=\mathrm{O}_{p}(1)$. Because $\sup _{\Theta \times \mathcal{N}\left(\xi_{0}\right)}\left|t_{n}(x \zeta, \xi) f(A(s \mid x \zeta, \delta))\right|$ is bounded and $a(s)$ is bounded by a polynomial of $s$, the uniform law of large numbers in Lemma 1 with $d=\bar{d}=0$ implies that as $n$ goes to infinity,

$$
\begin{aligned}
& \frac{1}{n} \sum_{i=1}^{n}\left\{t_{n}\left(x_{i} \zeta, \xi\right) a\left(s_{i}\right) f\left(A\left(s \mid x_{i} \zeta, \delta\right)\right)\right. \\
& \left.-\mathrm{E}\left[t_{n}\left(x_{i} \zeta, \xi\right) a\left(s_{i}\right) f\left(A\left(s \mid x_{i} \zeta, \delta\right)\right)\right]\right\} \stackrel{p}{\rightarrow} 0
\end{aligned}
$$

uniformly on $\Theta \times \mathscr{N}\left(\xi_{0}\right)$. The second part of the conclusion follows by the Lebesque dominated convergence theorem. Q.E.D.

Proposition 2. Let $\mathscr{N}\left(\xi_{0}\right)$ be a compact neighborhood of $\xi_{0}=\left(\xi_{p}\left(\delta_{0}\right), \xi_{(1-p)}\left(\delta_{0}\right)\right)$ such that for any $\xi=\left(\xi_{p}, \xi_{(1-p)}\right) \in \mathcal{N}\left(\xi_{0}\right), \quad\left[\xi_{p}, \xi_{(1-p)}\right] \subseteq \bar{T}$ where $\bar{T}=\left[\xi_{p}\left(\delta_{0}\right)-A, \xi_{(1-p)}\left(\delta_{0}\right)+\Delta\right]$ for some $\Delta>0$ (a constant not dependent on $\xi)$. Let $x \zeta=\left(x \zeta_{1}, x \zeta_{(2)}\right)$ and, conformably, $\xi=\left(\xi_{1}, \xi_{(2)}\right)$, where $x \zeta_{1}$ is a single index. Let $t_{n}\left(x \zeta_{(2)}, \xi_{(2)}\right)$ be the trimming function for the indices $x \zeta_{(2)}$ defined in 
Section A.3. Suppose that:

(1) $q$ is a continuously differentiable density function having a support $[0,1]$.

(2) $A_{n}\left(s \mid x_{i} \zeta\right) \stackrel{p}{\rightarrow} A\left(s \mid x_{i} \zeta, \delta\right)$ uniformly in $\left(x_{i} \zeta, \delta\right) \in \bar{T} \times \Theta$, where $\Theta$ is a compact neighborhood of $\delta_{0}$, and $A(s \mid x \zeta, \delta)$ is continuous in $\delta$ and $x \zeta$.

(3) $f(d)$ is uniformly continuous on $A_{\Delta}$, where $A_{\Delta}=\{d \mid d=A(s \mid x \zeta, \delta)$, $(x \zeta, \delta) \in \bar{T} \times \Theta\}$.

(4) a(s) is bounded by a polynomial of order $l, l \geqslant 0$. The $r \times l$-order moment of $s$ exists, where $r \geqslant 2$.

(5) The functions $q\left(\left(x_{\zeta_{1, p}}-\xi_{1, p}\right) / h_{n}\right) t_{n}\left(x \zeta_{(2)}, \xi_{(2)}\right)$ of $x \zeta$ have finite supports contained in $\bar{T}$ for all $\xi \in \mathcal{N}\left(\xi_{0}\right)$ for large $n$.

(6) $\mathrm{E}\left[t_{n}\left(x \zeta_{(2)}, \xi_{(2)}\right) a(s) f(A(s \mid x \zeta, \delta)) \mid x \zeta_{1}\right] g_{1}\left(x \zeta_{1} \mid \delta\right)$ is uniformly continuous at $x \zeta_{1}$, uniformly on $\Theta \times \mathscr{N}\left(\xi_{0}\right)$ and $n$, where $g_{1}(t \mid \delta)$ is the marginal density of $x \zeta_{1}$.

Then, under the rate that $\lim _{n \rightarrow \infty} h_{n}=0$ and $\lim _{n \rightarrow \infty} n h_{n}^{(1+2 l / r)} / \ln n=\infty$,

$$
\begin{aligned}
& \operatorname{plim}_{n \rightarrow \infty} \sup _{\Theta \times \mathcal{N}\left(\xi_{0}\right)} \mid \frac{1}{n} \sum_{i=1}^{n} \frac{1}{h_{n}} q\left(\frac{x_{i} \zeta_{1}-\xi_{1, p}}{h_{n}}\right) t_{n}\left(x_{i} \zeta_{(2)}, \xi_{(2)}\right) a\left(s_{i}\right) f\left(A_{n}\left(s \mid x_{i} \zeta\right)\right) \\
& -\mathrm{E}\left[t_{n}\left(x \zeta_{(2)}, \xi_{(2)}\right) a(s) f(A(s \mid x \zeta)) \mid x \zeta_{1}=\xi_{1, p}\right] g_{1}\left(\xi_{1, p} \mid \delta\right) \mid=0 .
\end{aligned}
$$

In addition to the above assumptions, if $\hat{\delta}$ is a consistent estimate of $\delta_{0}$ and $\hat{\xi}_{n}$ is a consistent estimate of $\xi_{0}$, then

$$
\begin{aligned}
& \operatorname{plim} \frac{1}{n} \sum_{i=1}^{n} \frac{1}{h_{n}} q\left(\frac{x_{i} \hat{\zeta}_{1}-\hat{\xi}_{1, p n}}{h_{n}}\right) t_{n}\left(x_{i} \hat{\zeta}_{(2)}, \xi_{(2), n}\right) a\left(s_{i}\right) f\left(A_{n}\left(s \mid x_{i} \hat{\zeta}\right)\right) \\
& =\mathrm{E}\left[I_{T_{(2)}}\left(x_{\zeta(2)}\left(\delta_{0}\right)\right) a(s) f\left(A\left(s \mid x \zeta_{0}\right)\right) \mid x \zeta_{1}\left(\delta_{0}\right)=\xi_{1, p}\left(\delta_{0}\right)\right] g_{1}\left(\xi_{1, p}\left(\delta_{0}\right) \mid \delta_{0}\right),
\end{aligned}
$$

where $T_{(2)}=\left[\xi_{(2), p}\left(\delta_{0}\right), \xi_{(2),(1-p)}\left(\delta_{0}\right)\right]$.

Proof

$$
\begin{aligned}
& \sup _{\Theta \times \mathcal{N}\left(\zeta_{0}\right)} \mid \frac{1}{n} \sum_{i=1}^{n} \frac{1}{h_{n}} q\left(\frac{x_{i} \zeta_{1}-\xi_{1, p}}{h_{n}}\right) t_{n}\left(x_{i} \zeta_{(2)}, \xi_{(2)}\right) \\
& \quad \times a\left(s_{i}\right)\left[f\left(A_{n}\left(s \mid x_{i} \zeta\right)\right)-f\left(A\left(s \mid x_{i} \zeta, \delta\right)\right)\right] \mid \\
& \leqslant \sup _{\Theta \times \mathcal{N}\left(\xi_{0}\right)} \frac{1}{n} \sum_{i=1}^{n}\left|a\left(s_{i}\right)\right| \frac{1}{h_{n}} q\left(\frac{x_{i} \zeta_{1}-\xi_{1, p}}{h_{n}}\right) \\
& \quad \times \sup _{\left(x_{i} \zeta, \delta\right) \in \bar{T} \times \Theta}\left|f\left(A_{n}\left(s \mid x_{i} \zeta\right)\right)-f\left(A\left(s \mid x_{i} \zeta, \delta\right)\right)\right| .
\end{aligned}
$$


By Lemma 1, as $n h_{n}^{(1+2 l / r)} / \ln n=\infty$,

$$
\sup _{\Theta \times \mathcal{N}\left(\xi_{0}\right)}\left|\frac{1}{n} \sum_{i=1}^{n}\right| a\left(s_{i}\right)\left|\frac{1}{h_{n}} q\left(\frac{x_{i} \zeta_{1}-\xi_{1, p}}{h_{n}}\right)-\mathrm{E}\left\{|a(s)| \frac{1}{h_{n}} q\left(\frac{x_{i} \zeta_{1}-\xi_{1, p}}{h_{n}}\right)\right\}\right| \stackrel{p}{\rightarrow} 0 .
$$

By Lemma 2,

$$
\begin{aligned}
& \sup _{\Theta \times \mathcal{N}\left(\zeta_{0}\right)}\left|\mathrm{E}\left\{|a(s)| \frac{1}{h_{n}} q\left(\begin{array}{c}
x \zeta_{1}-\xi_{1, p} \\
h_{n}
\end{array}\right)\right\}-\mathrm{E}\left(|a(s)| \mid x \zeta_{1}=\xi_{1, p}\right) g_{1}\left(\xi_{1, p} \mid \delta\right)\right| \\
& \rightarrow 0 .
\end{aligned}
$$

Since $\mathrm{E}\left(|a(s)| \mid x \zeta_{1}=\xi_{1, p}\right) g_{1}\left(\xi_{1, p} \mid \delta\right)$ is bounded on $\Theta \times \mathscr{N}\left(\xi_{0}\right)$, it follows that

$$
\sup _{\Theta \times \mathcal{N}\left(\xi_{0}\right)} \frac{1}{n} \sum_{i=1}^{n}\left|a\left(s_{i}\right)\right| \frac{1}{h_{n}} q\left(\frac{x_{i} \zeta_{1}-\xi_{1, p}}{h_{n}}\right)=\mathrm{O}_{p}(1),
$$

and hence $(*)$ goes to zero in probability. Similarly, by Lemma 1 ,

$$
\begin{aligned}
& \sup _{\Theta \times \mathcal{N}\left(\xi_{0}\right)} \mid \frac{1}{n} \sum_{i=1}^{n} \frac{1}{h_{n}} q\left(\frac{x_{i} \zeta_{1}-\xi_{1, p}}{h_{n}}\right) t_{n}\left(x_{i} \zeta_{(2)}, \xi_{(2)}\right) a\left(s_{i}\right) f\left(A\left(s \mid x_{i} \zeta, \delta\right)\right) \\
& -\mathrm{E}\left[\frac{1}{h_{n}} q\left(\frac{x \zeta_{1}-\xi_{1, p}}{h_{n}}\right) t_{n}\left(x \zeta_{(2)}, \xi_{(2)}\right) a(s) f(A(s \mid x \zeta, \delta))\right] \mid \stackrel{p}{\rightarrow} 0 .
\end{aligned}
$$

Since

$$
\begin{aligned}
& \mathrm{E}\left[\frac{1}{h_{n}} q\left(\frac{x \zeta_{1}-\xi_{1, p}}{h_{n}}\right) t_{n}\left(x \zeta_{(2)}, \xi_{(2)}\right) a(s) f(A(s \mid x \zeta, \delta))\right] \\
& =\int \mathrm{E}\left[t_{n}\left(x \zeta_{(2)}, \xi_{(2)}\right) a(s) f(A(s \mid x \zeta, \delta)) \mid x \zeta_{1}=\xi_{1, p}+h_{n} v\right] g_{1}\left(\xi_{1, p}+h_{n} v \mid \delta\right) q(v) \mathrm{d} v,
\end{aligned}
$$

it follows that

$$
\begin{aligned}
& \sup _{\Theta \times \mathcal{N}\left(\delta_{0}\right)} \mid \mathrm{E}\left[\frac{1}{h_{n}} q\left(\frac{x \zeta_{1}-\xi_{1, p}}{h_{n}}\right) t_{n}\left(x \zeta_{(2)}, \xi_{(2)}\right) a(s) f(A(s \mid x \zeta, \delta))\right] \\
& \quad-\mathrm{E}\left[t_{n}\left(x \zeta_{(2)}, \xi_{(2)}\right) a(s) f(A(s \mid x \zeta, \delta)) \mid x \zeta_{1}=\xi_{1, p}\right] g_{1}\left(\xi_{1, p} \mid \delta\right) \mid \\
& \leqslant \int_{\Theta \times \mathcal{N}\left(\delta_{0}\right), n} \mid \mathrm{E}\left[t_{n}\left(x \zeta_{(2)}, \xi_{(2)}\right) a(s) f(A(s \mid x \zeta, \delta)) \mid x \zeta_{1}=\xi_{1, p}+h_{n} v\right] \\
& \quad \times g_{1}\left(\xi_{1, p}+h_{n} v \mid \delta\right)-\mathrm{E}\left[t_{n}\left(x \zeta_{(2)}, \xi_{(2)}\right) a(s) f(A(s \mid x \zeta, \delta)) \mid x \zeta_{1}=\xi_{1, p}\right] \\
& \quad \times g_{1}\left(\xi_{1, p} \mid \delta\right) \mid q(v) \mathrm{d} v \\
& \rightarrow 0
\end{aligned}
$$


by the uniform continuity. The first part of the result follows from the above convergences. The second part of the result follows by the Lebesque dominated convergence theorem. Q.E.D.

Lemma 5. Let $C_{n}\left(\bar{s}_{1}, \ldots, \bar{s}_{i-1}, \bar{s}_{i+1}, \ldots, \bar{s}_{n} ; \bar{s}_{i}\right)$ be a sequence of measurable functions of an i.i.d. sample $\left\{\bar{s}_{i}\right\}$ and $d_{n}(\bar{s})$ be a measurable function such that $\mathrm{E}\left(\left|d_{n}(\bar{s})\right|\right)<\infty$ uniformly in $n$. Suppose that:

(l) $\sup \left|\mathrm{E}\left(C_{n}\left(\bar{s}_{1}, \cdots, \bar{s}_{n} ; \bar{s}_{i}\right) \mid \bar{s}_{i}\right)-C\left(\bar{s}_{i}\right)\right|=\mathrm{O}\left(a_{n}^{s^{*}}\right)$, for some measurable function $C\left(\bar{s}_{i}\right)$, and

(2) $\sup \operatorname{var}\left(C_{n}\left(\bar{s}_{1}, \cdots, \bar{s}_{n} ; \bar{s}_{i}\right) \mid \bar{s}_{i}\right)=\mathrm{O}\left(1 / n a_{n}^{r}\right)$.

If $s^{*}>r / 2, \lim _{n \rightarrow \infty} n a_{n}^{2 r}=\infty$, and $\lim _{n \rightarrow \infty} n a_{n}^{4 s^{*}}=0$, then

$$
\frac{1}{\sqrt{n}} \sum_{i=1}^{n} d_{n}\left(\bar{s}_{i}\right)\left|C_{n}\left(\bar{s}_{1}, \ldots, \bar{s}_{n} ; \bar{s}_{i}\right)-C\left(\bar{s}_{i}\right)\right|^{2} \stackrel{p}{\rightarrow} 0 .
$$

Proof. This result can be easily proved by the Markov inequality (Lee, 1992, p. 79, Lemma 6).

Proposition 3. Let $\nabla_{a} f_{n}(s, a)$ and $\nabla_{a}^{2} f_{n}(s, a)$ denote the first-and second-order derivatives of $f_{n}(s, a)$ with respect to a. Suppose that:

(I) The nonparametric function $A_{n}\left(s \mid x_{i} \zeta_{0}\right)$ satisfies the conditions in Lemma 5 , and

(2) $\sup _{x_{i} \zeta_{0} \in T}\left|\nabla_{a}^{2} f_{n}\left(s_{i}, \bar{A}_{n}\left(s \mid x_{i} \zeta_{0}\right)\right)\right| \leqslant \mathrm{O}_{p}(1) d_{n}\left(s_{i}\right)$, where $\bar{A}_{n}\left(s \mid x_{i} \zeta_{0}\right)$ is a consistent estimate of $A\left(s \mid x_{i} \zeta_{0}, \delta_{0}\right)$ and $d_{n}\left(s_{i}\right)$ is a measurable function such that $\mathrm{E}\left(I_{T}\left(x_{i} \zeta_{0}\right) d_{n}\left(s_{i}\right)\right)<\infty$ uniformly in $n$.

Then

$$
\begin{aligned}
& \frac{1}{\sqrt{n}} \sum_{i=1}^{n} I_{T}\left(x_{i} \zeta_{0}\right) f_{n}\left(s_{i}, A_{n}\left(s \mid x_{i} \zeta_{0}\right)\right) \\
& =\frac{1}{\sqrt{n}} \sum_{i=1}^{n} I_{T}\left(x_{i} \zeta_{0}\right) f_{n}\left(s_{i}, A\left(s \mid x_{i} \zeta_{0}, \delta_{0}\right)\right) \\
& \quad+\frac{1}{\sqrt{n}} \sum_{i=1}^{n} I_{T}\left(x_{i} \zeta_{0}\right) \nabla_{a} f_{n}\left(s_{i}, A\left(s \mid x_{i} \zeta_{0}, \delta_{0}\right)\right) \\
& \quad \times\left(A_{n}\left(s \mid x_{i} \zeta_{0}\right)-A\left(s \mid x_{i} \zeta_{0}, \delta_{0}\right)\right)+o_{p}(1) .
\end{aligned}
$$

Proof. By a Taylor expansion,

$$
\begin{aligned}
& \frac{1}{\sqrt{n}} \sum_{i=1}^{n} I_{T}\left(x_{i} \zeta_{0}\right) f_{n}\left(s_{i}, A_{n}\left(s \mid x_{i} \zeta_{0}\right)\right) \\
& =\frac{1}{\sqrt{n}} \sum_{i=1}^{n} I_{T}\left(x_{i} \zeta_{0}\right) f_{n}\left(s_{i}, A\left(s \mid x_{i} \zeta_{0}, \delta_{0}\right)\right)+L_{n}+R_{n},
\end{aligned}
$$


where

$$
L_{n}=\frac{1}{\sqrt{n}} \sum_{i=1}^{n} I_{T}\left(x_{i} \zeta_{0}\right) \nabla_{a} f_{n}\left(s_{i}, A\left(s \mid x_{i} \zeta_{0}, \delta_{0}\right)\right)\left(A_{n}\left(s \mid x_{i} \zeta_{0}\right)-A\left(s \mid x_{i} \zeta_{0}, \delta_{0}\right)\right)
$$

and

$$
R_{n}=\frac{1}{2} \frac{1}{\sqrt{n}} \sum_{i=1}^{n} I_{T}\left(x_{i} \zeta_{0}\right) \nabla_{a}^{2} f_{n}\left(s_{i}, \bar{A}_{n}\left(s \mid x_{i} \zeta_{0}\right)\right)\left(A_{n}\left(s \mid x_{i} \zeta_{0}\right)-A\left(s \mid x_{i} \zeta_{0}, \delta_{0}\right)\right)^{2},
$$

where $\overline{A_{n}}\left(s \mid x_{i} \zeta_{0}\right)$ lies between $A_{n}\left(s \mid x_{i} \zeta_{0}\right)$ and $A\left(s \mid x_{i} \zeta_{0}, \delta_{0}\right)$. Since

$$
\left|R_{n}\right| \leqslant \mathrm{O}_{p}(1) \frac{1}{\sqrt{n}} \sum_{i=1}^{n} I_{T}\left(x_{i} \zeta_{0}\right) d_{n}\left(s_{i}\right)\left[A_{n}\left(s \mid x_{i} \zeta_{0}\right)-A\left(s \mid x_{i} \zeta_{0}, \delta_{0}\right)\right]^{2}
$$

by our assumption, Lemma 5 implies that $R_{n}$ converges to zero in probability. Q.E.D.

Lemma 6. Let $\left\{\bar{s}_{i}\right\}$ be an i.i.d. sample and $\Phi_{n}\left(\bar{s}_{1}, \bar{s}_{2}, a_{n}\right)$ be a sequence of vectorvalued random functions with bandwidth $a_{n}$. Suppose that:

(1) There exist square integrable functions $h_{j}(\bar{s})$ such that $\left|\mathrm{E}\left(\Phi_{n}\left(\bar{s}_{1}, \bar{s}_{2}, a_{n}\right) \mid \bar{s}_{j}\right)\right|$ $\leqslant h_{j}\left(\bar{s}_{j}\right)$ for $j=1,2$.

(2) $\mathrm{E}\left(\Phi_{n}\left(\bar{s}_{1}, \bar{s}_{2}, a_{n}\right)\right)=\mathrm{O}\left(a_{n}^{s^{*}}\right)$ and $\operatorname{var}\left(\Phi_{n}\left(\bar{s}_{1}, \bar{s}_{2}, a_{n}\right)\right)=\mathrm{O}\left(1 / a_{n}^{r}\right)$.

(3) $\lim _{n \rightarrow \infty} \mathrm{E}\left(\Phi_{n}\left(\bar{s}_{1}, \bar{s}_{2}, a_{n}\right) \mid \bar{s}_{j}\right)=\psi_{j}\left(\bar{s}_{j}\right)$, a.e., for some measurable functions $\psi_{j}$, $j=1,2$.

(4) $\lim _{n \rightarrow \infty} \sqrt{n} a_{n}^{s^{*}}=0$ and $\lim _{n \rightarrow \infty} n a_{n}^{r}=\infty$.

If $\psi_{1}(\bar{s})$ and $\psi_{2}(\bar{s})$ are zero a.e., then

$$
\frac{1}{\sqrt{n}(n-1)} \sum_{i=1}^{n} \sum_{j \neq i}^{n} \Phi_{n}\left(\bar{s}_{i}, \bar{s}_{j}, a_{n}\right) \stackrel{p}{\rightarrow} 0 .
$$

On the other hand, if $\mathrm{E}\left\{\left[\psi_{1}(\bar{s})+\psi_{2}(\bar{s})\right]\left[\psi_{1}(\bar{s})+\psi_{2}(\bar{s})\right]^{\prime}\right\}=\Sigma$ which is nonzero, then

$$
\frac{1}{\sqrt{n}(n-1)} \sum_{i=1}^{n} \sum_{j \neq i}^{n} \Phi_{n}\left(\bar{s}_{i}, \bar{s}_{j}, a_{n}\right) \stackrel{D}{\rightarrow} \mathrm{N}(0, \Sigma) .
$$

Proof. These results follow from Powell, Stock and Stoker (1989) and the Lindeberg central limit theorem. Q.E.D.

Proposition 4. Suppose that $K$ is an $r$-dimensional kernel function with a bounded support $D$ such that $\int_{D}|K(v)| \mathrm{d} v<\infty$ and with a bandwidth $a_{n}$. Let

$$
A_{n}\left(s \mid x_{i} \zeta_{0}\right)=\frac{1}{(n-1) a_{n}^{r}} \sum_{j \neq i}^{n} s_{j} K\left(\frac{x_{i} \zeta_{0}-x_{j} \zeta_{0}}{a_{n}}\right),
$$


and let $g\left(x \zeta_{0} \mid \delta_{0}\right)$ be the density of $x \zeta_{0}$. Denote $A\left(s \mid x \zeta_{0}, \delta_{0}\right)=\mathrm{E}\left(s \mid x \zeta_{0}\right) g\left(x \zeta_{0} \mid \delta_{0}\right)$. Let $f_{n}\left(s, x \zeta_{0}\right)$ be measurable functions such that $\sup _{n}\left|f_{n}\left(s, x \zeta_{0}\right)\right|$ is square integrable. Suppose that:

(1) $\mathrm{E}\left(s \mid x \zeta_{0}\right) g\left(x \zeta_{0} \mid \delta_{0}\right)$ is uniformly continuous at $x \zeta_{0}$, and

(2) $\mathrm{E}\left(f_{n}\left(s, x \zeta_{0}\right) \mid x \zeta_{0}\right)=0$ a.e., for all $n$.

If $\lim _{n \rightarrow \infty} n a_{n}^{r}=\infty$, then

$$
\frac{1}{\sqrt{n}} \sum_{i=1}^{n} f_{n}\left(s_{i}, x_{i} \zeta_{0}\right)\left[A_{n}\left(s \mid x_{i} \zeta_{0}\right)-A\left(s \mid x_{i} \zeta_{0}, \delta_{0}\right)\right] \stackrel{p}{\rightarrow} 0 .
$$

Proof. Define

$$
\Phi_{n}\left(\bar{s}_{i}, \bar{s}_{j}, a_{n}\right)=f_{n}\left(s_{i}, x_{i} \zeta_{0}\right)\left[\frac{1}{a_{n}^{r}} s_{j} K\left(\frac{x_{i} \zeta_{0}-x_{j} \zeta_{0}}{a_{n}}\right)-A\left(s \mid x_{i} \zeta, \delta_{0}\right)\right],
$$

where $\bar{s}=(s, x)$. It follows that

$$
\begin{aligned}
& \frac{1}{\sqrt{n}} \sum_{i=1}^{n} f_{n}\left(s_{i}, \mathrm{x}_{i} \zeta_{0}\right)\left[A_{n}\left(s \mid x_{i} \zeta_{0}\right)-A\left(s \mid x_{i} \zeta_{0}, \delta_{0}\right)\right] \\
& =\frac{1}{(n-1) \sqrt{n}} \sum_{i=1}^{n} \sum_{j \neq i}^{n} \Phi_{n}\left(\bar{s}_{i}, \bar{s}_{j}, a_{n}\right) .
\end{aligned}
$$

For any $\varepsilon>0$, for large $n$ Lemma 2 implies that

$$
\begin{aligned}
& \left|\mathrm{E}\left(\Phi_{n}\left(\bar{s}_{1}, \bar{s}_{2}, a_{n}\right) \mid \bar{s}_{1}\right)\right| \\
& \leqslant\left|f_{n}\left(s_{1}, x_{1} \zeta_{0}\right)\right|\left|\mathrm{E}\left[\frac{1}{a_{n}^{r}} s_{2} K\left(\frac{x_{1} \zeta_{0}-x_{2} \zeta_{0}}{a_{n}}\right)-A\left(s \mid x_{1} \zeta, \delta_{0}\right) \mid x_{1}\right]\right| \\
& \leqslant \varepsilon \sup \left|f_{n}\left(s_{1}, x_{1} \zeta_{0}\right)\right|,
\end{aligned}
$$

which is square integrable. Since $\varepsilon$ is arbitary, the above relations imply that $\mathrm{E}\left(\Phi_{n}\left(s_{i}, s_{j}, a_{n}\right) \mid s_{i}\right)$ converges to zero. On the other hand,

$$
\begin{aligned}
& \mathrm{E}\left(\Phi_{n}\left(\bar{s}_{j}, \bar{s}_{i}, a_{n}\right) \mid \bar{s}_{i}\right) \\
&=s_{i} \mathrm{E}\left[f_{n}\left(s_{j}, x_{j} \zeta_{0}\right) \frac{1}{a_{n}^{r}} K\left(\frac{x_{j} \zeta_{0}-x_{i} \zeta_{0}}{a_{n}}\right) \mid x_{i}\right]-\mathrm{E}\left[f_{n}\left(s_{j}, x_{j} \zeta_{0}\right) A\left(s \mid x_{j} \zeta_{0}, \delta_{0}\right)\right] \\
&=s_{i} \int \mathrm{E}\left[f_{n}\left(s, x_{i} \zeta_{0}+a_{n} v\right) \mid x_{j} \zeta_{0}=x_{i} \zeta_{0}+a_{n} v, x_{i}\right] g\left(x_{i} \zeta_{0}+a_{n} v \mid \delta_{0}\right) K(v) \mathrm{d} v \\
&\left.-\mathrm{E}\left\{\mathrm{E}\left[f_{n}\left(s, x \zeta_{0}\right) \mid x \zeta_{0}\right)\right] A\left(s \mid x \zeta_{0}, \delta_{0}\right)\right\} \\
&= 0,
\end{aligned}
$$


by the condition (2). The conclusion of the proposition follows from Lemma 6. Q.E.D.

Proposition 5. Suppose that $K$ is an $r$-dimensional kernel function with a bounded support $D$ such that $\int_{D}|K(v)| \mathrm{d} v<\infty$ and with a bandwidth $a_{n}$. Let

$$
A_{n}\left(s \mid x_{i} \zeta_{0}\right)=\frac{1}{(n-1) a_{n}^{r}} \sum_{j \neq i}^{n} s_{j} K\left(\frac{x_{i} \zeta_{0}-x_{j} \zeta_{0}}{a_{n}}\right),
$$

and let $g\left(x \zeta_{0} \mid \delta_{0}\right)$ be the density of $x \zeta_{0}$. Denote $A\left(s \mid x \zeta_{0}, \delta_{0}\right)=\mathrm{E}\left(s \mid x \zeta_{0}\right) \times$ $g\left(x \zeta_{0} \mid \delta_{0}\right)$. Let $f_{n}\left(s, x \zeta_{0}\right)$ be measurable functions such that $\sup _{n}\left|f_{n}\left(s, x \zeta_{0}\right)\right|$ is square integrable. Suppose that:

(1) $\mathrm{E}\left(s \mid x \zeta_{0}\right) g\left(x \zeta_{0} \mid \delta_{0}\right)$ is uniformly continuous at $x \zeta_{0}$.

(2) $\mathrm{E}\left(f_{n}\left(s, x \zeta_{0}\right) \mid x \zeta_{0}\right) g\left(x \zeta_{0} \mid \delta_{0}\right)$ is continuous in $x \zeta_{0}$ a.e. uniformly in $n$.

(3) There exists a measurable function $h\left(x \zeta_{0}\right)$ such that $\left|\mathrm{E}\left(f_{n}\left(s, x \zeta_{0}\right) \mid x \zeta_{0}\right)\right|$ $\leqslant h\left(x \zeta_{0}\right)$, with $\mathrm{E}\left|h\left(x \zeta_{0}\right) A\left(s \mid x \zeta_{0}, \delta_{0}\right)\right|<\infty$ for large $n$.

(4) $\lim _{n \rightarrow \infty} \mathrm{E}\left(f_{n}\left(s, x \zeta_{0}\right) \mid x \zeta_{0}\right)=c\left(x \zeta_{0}\right)$ a.e.

$$
\begin{aligned}
& \text { If } \lim _{n \rightarrow \infty} n a_{n}^{r}=\infty \text { and } \lim _{n \rightarrow \infty} n a_{n}^{2 s}=0, \text { then } \\
& \qquad \frac{1}{\sqrt{n}} \sum_{i=1}^{n} f_{n}\left(s_{i}, x_{i} \zeta_{0}\right)\left[A_{n}\left(s \mid x_{i} \zeta_{0}\right)-A\left(s \mid x_{i} \zeta_{0}, \delta_{0}\right)\right] \stackrel{D}{\rightarrow} \mathrm{N}(0, \Phi),
\end{aligned}
$$

where

$$
\begin{aligned}
\Phi= & \mathrm{E}\left\{\left[s \cdot c\left(x \zeta_{0}\right) g\left(x \zeta_{0} \mid \delta_{0}\right)-\mathrm{E}\left(s \cdot c\left(x \zeta_{0}\right) g\left(x \zeta_{0} \mid \delta_{0}\right)\right)\right]^{\prime}\right. \\
& \left.\times\left[s \cdot c\left(x \zeta_{0}\right) g\left(x \zeta_{0} \mid \delta_{0}\right)-\mathrm{E}\left(s \cdot c\left(x \zeta_{0}\right) g\left(x \zeta_{0} \mid \delta_{0}\right)\right)\right]\right\}
\end{aligned}
$$

Proof.

$$
\frac{1}{\sqrt{n}} \sum_{i=1}^{n} f_{n}\left(s_{i}, x_{i} \zeta_{0}\right)\left[A_{n}\left(s \mid x_{i} \zeta_{0}\right) \quad A\left(s \mid x_{i} \zeta_{0}, \delta_{0}\right)\right]=\frac{1}{(n-1) \sqrt{n}} \sum_{i=1}^{n} \sum_{j \neq i}^{n} \Phi_{n}\left(s_{i}, s_{j}, a_{n}\right),
$$

where

$$
\Phi_{n}\left(\bar{s}_{i}, \bar{s}_{j}, a_{n}\right)=f_{n}\left(s_{i}, x_{i} \zeta_{0}\right)\left[\frac{1}{a_{n}^{r}} s_{j} K\left(\frac{x_{i} \zeta_{0}-x_{j} \zeta_{0}}{a_{n}}\right)-A\left(s \mid x_{i} \zeta, \delta_{0}\right)\right] .
$$

By (1), $\mathrm{E}\left(\Phi_{n}\left(\bar{s}_{i}, \bar{s}_{j}, a_{n}\right) \mid \bar{s}_{i}\right)$ converges to zero. On the other hand,

$$
\begin{aligned}
\mathrm{E}\left(\Phi_{n}\left(\bar{s}_{j}, \bar{s}_{i}, a_{n}\right) \mid \bar{s}_{i}\right)= & s_{i} \mathrm{E}\left[f_{n}\left(s_{j}, x_{j} \zeta_{0}\right) \frac{1}{a_{n}^{r}} K\left(\frac{x_{j} \zeta_{0}-x_{i} \zeta_{0}}{a_{n}}\right) \mid x_{i}\right] \\
& -\mathrm{E}\left\{\mathrm{E}\left[f_{n}\left(s, x \zeta_{0}\right) \mid x \zeta_{0}\right] A\left(s \mid x \zeta_{0}, \delta_{0}\right)\right\} .
\end{aligned}
$$


Since

$$
\begin{aligned}
& \lim _{n \rightarrow \infty}\left\{\mathrm{E}\left[f_{n}\left(s_{j}, x_{j} \zeta_{0}\right) \frac{1}{a_{n}^{r}} K\left(\frac{x_{j} \zeta_{0}-x_{i} \zeta_{0}}{a_{n}}\right) \mid x_{i}\right]\right. \\
& \left.-\mathrm{E}\left[f_{n}\left(s, x \zeta_{0}\right) \mid x \zeta_{0}=x_{i} \zeta_{0}\right] g\left(x_{i} \zeta_{0} \mid \delta_{0}\right)\right\}=0
\end{aligned}
$$

by condition (2), it follows that, by conditions (3) and (4) and the Lebesque convergence theorem,

$$
\lim _{n \rightarrow \infty} \mathrm{E}\left(\Phi_{n}\left(\bar{s}_{j}, \bar{s}_{i}, a_{n}\right) \mid \bar{s}_{i}\right)=s_{i} c\left(x_{i} \zeta_{0}\right) g\left(x_{i} \zeta_{0} \mid \delta_{0}\right)-\mathrm{E}\left(c\left(x \zeta_{0}\right) \mathrm{E}\left(s \mid x \zeta_{0}\right) g\left(x \zeta_{0} \mid \delta_{0}\right)\right) \text { a.e. }
$$

The result of the proposition follows from the second part of Lemma 6. Q.E.D.

Proposition 6. Suppose that $K$ is an $r$-dimensional kernel function with a bounded support $D$ such that $\int_{D}|K(v)| \mathrm{d} v<\infty$ and with a bandwidth $a_{n}$. Let

$$
D_{n}\left(s \mid x_{i} \zeta_{0}\right)=\frac{1}{(n-1) a_{n}^{r+1}} \sum_{j \neq i}^{n} s_{j} \nabla K\left(\frac{x_{i} \zeta_{0}-x_{j} \zeta_{0}}{a_{n}}\right),
$$

where $\nabla K(v)=\partial K(v) / \partial v$ is the gradient vector of $K$, and let $g\left(x \zeta_{0} \mid \delta_{0}\right)$ be the density of $x \zeta_{0}$. Denote

$$
D\left(s \mid x \zeta_{0}, \delta_{0}\right)=\frac{\partial}{\partial\left(x \zeta_{0}\right)}\left\{\mathrm{E}\left(s \mid x \zeta_{0}\right) g\left(x \zeta_{0} \mid \delta_{0}\right)\right\} .
$$

Let $f_{n}\left(s, x \zeta_{0}\right)$ be measurable functions such that $\sup _{n}\left|f_{n}\left(s, x \zeta_{0}\right)\right|$ is square integrable. Suppose that:

(I) $K(v)$ vanishes at the boundary of $D$.

(2) $\left(\partial / \partial\left(x \zeta_{0}\right)\right)\left\{\mathrm{E}\left(s \mid x \zeta_{0}\right) g\left(x \zeta_{0} \mid \delta_{0}\right)\right\}$ is uniformly continuous at $x \zeta_{0}$.

(3) $\mathrm{E}\left(f_{n}\left(s, x \zeta_{0}\right) \mid x \zeta_{0}\right)=0$ a.e., for all $n$.

If $\lim _{n \rightarrow \infty} n a_{n}^{r+2}=\infty$, then

$$
\frac{1}{\sqrt{n}} \sum_{i=1}^{n} f_{n}\left(s_{i}, x_{i} \zeta_{0}\right)\left[D_{n}\left(s \mid x_{i} \zeta_{0}\right)-D\left(s \mid x_{i} \zeta_{0}, \delta_{0}\right)\right] \stackrel{p}{\rightarrow} 0 .
$$

Proof. Define

$$
\Phi_{n}\left(\bar{s}_{i}, \bar{s}_{j}, a_{n}\right)=f_{n}\left(s_{i}, x_{i} \zeta_{0}\right)\left[\frac{1}{a_{n}^{r+1}} s_{j} \nabla K\left(\frac{x_{i} \zeta_{0}-x_{j} \zeta_{0}}{a_{n}}\right)-D\left(s \mid x_{i} \zeta_{0}, \delta_{0}\right)\right] .
$$

It follows that

$$
\begin{aligned}
& \frac{1}{\sqrt{n}} \sum_{i=1}^{n} f_{n}\left(s_{i}, x_{i} \zeta_{0}\right)\left[D_{n}\left(s \mid x_{i} \zeta_{0}\right)-D\left(s \mid x_{i} \zeta_{0}, \delta_{0}\right)\right] \\
& -\frac{1}{(n-1) \sqrt{n}} \sum_{i=1}^{n} \sum_{j \neq 1}^{n} \Phi_{n}\left(\bar{s}_{i}, \bar{s}_{j}, a_{n}\right) .
\end{aligned}
$$


By conditions (1) and (2), Lemma 3 implies that

$$
\mathrm{E}\left[s_{j} \frac{1}{a_{n}^{r+1}} \nabla K\left(\frac{x_{i} \zeta_{0}-x_{j} \zeta_{0}}{a_{n}}\right) \mid x_{i}\right] \text { converges to } D\left(s \mid x_{i} \zeta_{0}, \delta_{0}\right) \text {. }
$$

Hence $\mathrm{E}\left(\Phi_{n}\left(\bar{s}_{i}, \bar{s}_{j}, a_{n}\right) \mid \bar{s}_{i}\right)$ converges to zero. On the other hand, under condition (3),

$$
\begin{aligned}
\mathrm{E}\left(\Phi_{n}\left(\bar{s}_{j}, \bar{s}_{i}, a_{n}\right) \mid \bar{s}_{i}\right)= & s_{i} \mathrm{E}\left[f_{n}\left(s_{j}, x_{j} \zeta_{0}\right) \frac{1}{a_{n}^{r+1}} \nabla K\left(\frac{x_{j} \zeta_{0}-x_{i} \zeta_{0}}{a_{n}}\right) \mid x_{i}\right] \\
& -\mathrm{E}\left[f_{n}\left(s_{j}, x_{j} \zeta_{0}\right) D\left(s \mid x_{j} \zeta_{0}, \delta_{0}\right)\right] \\
= & s_{i} \frac{1}{a_{n}} \int_{D} \mathrm{E}\left[f_{n}\left(s, x_{i} \zeta_{0}+a_{n} v\right) \mid x_{j} \zeta_{0}=x_{i} \zeta_{0}+a_{n} v, x_{i}\right] \\
& \times g\left(x_{i} \zeta_{0}+a_{n} v \mid \delta_{0}\right) \nabla K(v) \mathrm{d} v \\
& -\mathrm{E}\left\{\mathrm{E}\left[f_{n}\left(s, x \zeta_{0}\right) \mid x \zeta_{0}\right] D\left(s \mid x \zeta_{0}, \delta_{0}\right)\right\} \\
= & 0 .
\end{aligned}
$$

The result of the proposition follows from the first part of Lemma 6. Q.E.D.

\section{A.5. Asymptotic properties}

The asymptotic distributions of

$$
\frac{1}{\sqrt{n}} \hat{X}_{2}^{\prime} \hat{U}_{n}, \quad \frac{1}{\sqrt{n}} \hat{X}_{2}^{\prime} \hat{\Lambda}_{n}^{-1} \hat{U}_{n}, \quad \frac{1}{\sqrt{n}} \frac{\partial \mathrm{E}_{n}(\hat{\beta}, \hat{\zeta})}{\partial \delta} \hat{\Lambda}_{n}^{-1} \hat{U}_{n}
$$

can be derived from Propositions 1-6.

$$
\begin{aligned}
\frac{1}{\sqrt{n}} \hat{X}_{2}^{\prime} \hat{U}_{n} & =\frac{1}{\sqrt{n}} \sum_{i=1}^{n} t_{n}\left(x_{i} \hat{\zeta}, \hat{\xi}_{n}\right)\left(x_{(2) i}-\mathrm{E}_{n}\left(x_{(2)} \mid x_{i} \hat{\zeta}\right)\right)^{\prime}\left(u_{1 i}-\mathrm{E}_{n}\left(u_{1} \mid x_{i} \hat{\zeta}\right)\right) \\
& =C_{0, n}+\left\{C_{1, n}+C_{2, n}+C_{3, n}\right\} \sqrt{n}\left(\hat{\delta}-\delta_{0}\right)+C_{4, n} \sqrt{n}\left(\hat{\xi}_{n}-\xi_{0}\right)
\end{aligned}
$$

where

$$
\begin{aligned}
& C_{0, n}=\frac{1}{\sqrt{n}} \sum_{i=1}^{n} t_{n}\left(x_{i} \zeta_{0}, \xi_{0}\right)\left(x_{(2) i}-\mathrm{E}_{n}\left(x_{(2)} \mid x_{i} \zeta_{0}\right)\right)^{\prime}\left(u_{1 i}-\mathrm{E}_{n}\left(u_{1} \mid x_{i} \zeta_{0}\right)\right), \\
& C_{1, n}=-\frac{1}{n} \sum_{i=1}^{n} t_{n}\left(x_{i} \bar{\zeta}, \bar{\xi}\right)\left(x_{(2) i}-\mathrm{E}_{n}\left(x_{(2)} \mid x_{i} \bar{\zeta}\right)\right)^{\prime} \frac{\partial \mathrm{E}_{n}\left(u_{1} \mid x_{i} \bar{\zeta}\right)}{\partial \delta^{\prime}} \\
& C_{2, n}=-\frac{1}{n} \sum_{i=1}^{n} t_{n}\left(x_{i} \bar{\zeta}, \bar{\zeta}\right)\left(u_{1 i}-\mathrm{E}_{n}\left(u_{1} \mid x_{i} \bar{\zeta}\right)\right)^{\prime} \frac{\partial \mathrm{E}_{n}\left(x_{2}^{\prime} \mid x_{i} \bar{\zeta}\right)}{\partial \delta^{\prime}} \\
& C_{3, n}=\frac{1}{n} \sum_{i=1}^{n}\left(x_{(2) i}-\mathrm{E}_{n}\left(x_{(2)} \mid x_{i} \bar{\zeta}\right)\right)^{\prime}\left(u_{1 i}-\mathrm{E}_{n}\left(u_{1} \mid x_{i} \bar{\zeta}\right)\right) \frac{\partial t_{n}\left(x_{i} \bar{\zeta}, \bar{\zeta}\right)}{\partial \delta^{\prime}}
\end{aligned}
$$




$$
C_{1, n}=\frac{1}{n} \sum_{i=1}^{n}\left(x_{(2) i}-\mathrm{E}_{n}\left(x_{(2)} \mid x_{i} \bar{\zeta}\right)\right)^{\prime}\left(u_{1 i}-\mathrm{E}_{n}\left(u_{1} \mid x_{i} \bar{\zeta}\right)\right) \frac{\partial t_{n}\left(x_{i} \bar{\zeta}, \bar{\zeta}\right)}{\partial \xi^{\prime}}
$$

Proposition 1 implies that

$$
C_{1, n} \stackrel{p}{\rightarrow}-\mathrm{E}\left(I_{T}\left(x \zeta_{0}\right)\left(x_{(2)}-\mathrm{E}\left(x_{(2)} \mid x \zeta_{0}\right)\right)^{\prime} \frac{\partial \mathrm{E}\left(u_{1} \mid x \zeta_{0}, \delta_{0}\right)}{\partial \delta^{\prime}}\right),
$$

and $C_{2, n}$ converges to zero in probability. As $\lim _{n \rightarrow \infty}(n / \ln n) h_{n}^{(1+4 / r)}=\infty$, Proposition 2 implies that $C_{3, n}$ and $C_{4, n}$ converge to zero in probability. To see the latter, consider the single-index model for simplicity. From (A.3.2), since $q$ has support on $[0,1]$ and is vanishing outside $[0,1]$, for large $n(A .3 .2)$ can be rewritten as

$$
\frac{\partial t_{n}(x \zeta, \zeta)}{\partial \delta^{\prime}}=\frac{1}{h_{n}} q\left(\frac{x \zeta-\xi_{p}}{h_{n}}\right) x_{(2)}-\frac{1}{h_{n}} q\left(\frac{x \zeta-\xi_{(1-p)}+h_{n}}{h_{n}}\right) x_{(2)} ;
$$

(A.3.3) can be written as

$$
\frac{\partial t_{n}(x \zeta, \zeta)}{\partial \xi_{p}}=-\frac{1}{h_{n}} q\left(\frac{x \zeta-\zeta_{p}}{h_{n}}\right)
$$

and (A.3.4) is

$$
\frac{\partial t_{n}(x \zeta, \xi)}{\partial \xi_{(1-p)}}=\frac{1}{h_{n}} q\left(\frac{x \zeta-\xi_{(1-p)}+h_{n}}{h_{n}}\right) .
$$

Hence $C_{3, n}=C_{3, n}^{(1)}-C_{3, n}^{(2)}$ where

$$
C_{3, n}^{(1)}=\frac{1}{n} \sum_{i=1}^{n} \frac{1}{h_{n}} q\left(\frac{x_{i} \bar{\zeta}-\bar{\xi}_{p}}{h_{n}}\right)^{\prime}\left(u_{1 i}-\mathrm{E}_{n}\left(u_{1} \mid x_{i} \bar{\zeta}\right)\right)\left(x_{(2) i}-\mathrm{E}_{n}\left(x_{(2)} \mid x_{i} \bar{\zeta}\right)\right)^{\prime} x_{(2) i},
$$

and

$$
\begin{aligned}
C_{3, n}^{(2)}= & -\frac{1}{n} \sum_{i=1}^{n} \frac{1}{h_{n}} q\left(\frac{x_{i} \bar{\zeta}-\bar{\xi}_{(1 p)}+h_{n}}{h_{n}}\right) \\
& \times\left(u_{1 i}-\mathrm{E}_{n}\left(u_{1} \mid x_{i} \bar{\zeta}\right)\right)\left(x_{(2) i}-\mathrm{E}_{n}\left(x_{(2)} \mid x_{i} \bar{\zeta}\right)\right)^{\prime} x_{(2)} i
\end{aligned}
$$

By the equivalent expressions for (A.3.3) and (A.3.4) above, $C_{4, n}$ has a similar expression. Proposition 2 is applicable to these terms. For example,

$$
\begin{aligned}
\operatorname{plim}_{n \rightarrow \infty} C_{3, n}^{(1)} & =\left.\mathrm{E}\left[\left(u_{1}-\mathrm{E}\left(u_{1} \mid x \zeta_{0}\right)\right)\left(x_{(2)}-\mathrm{E}\left(x_{(2)} \mid x \zeta_{0}\right)\right)^{\prime} x_{(2)} \mid x \zeta_{0}\right] p\left(x \zeta_{0} \mid \delta_{0}\right)\right|_{x \zeta_{0}=\xi_{p}\left(\delta_{0}\right)} \\
& =\left.\mathrm{E}\left[\left(u_{1}-\mathrm{E}\left(u_{1} \mid x \zeta_{0}\right)\right)\left(x_{(2)}-\mathrm{E}\left(x_{(2)} \mid x \zeta_{0}\right)\right)^{\prime} x_{(2)} \mid x \zeta_{0}\right] p\left(x \zeta_{0} \mid \delta_{0}\right)\right|_{x \zeta_{0}=\zeta_{p}\left(\delta_{0}\right)} \\
& =0
\end{aligned}
$$


because $\mathrm{E}\left(u_{1}-\mathrm{E}\left(u_{1} \mid x \zeta_{0}\right) \mid x\right)=0$. Thus, both $C_{3, n}$ and $C_{4, n}$ converge to zero in probability.

Consider the term $C_{0, n}$. To simplify notations, let

$$
\begin{aligned}
A_{n i} & =\frac{1}{(n-1) a_{n}^{m}} \sum_{j \neq i}^{n} x_{2 j} K\left(\frac{x_{i} \zeta_{0}-x_{j} \zeta_{0}}{a_{n}}\right), \\
B_{n i} & =\frac{1}{(n-1) a_{n}^{m}} \sum_{j \neq i}^{n} K\left(\frac{x_{i} \zeta_{0}-x_{j} \zeta_{0}}{a_{n}}\right), \\
C_{n i} & =\frac{1}{(n-1) a_{n}^{m}} \sum_{j \neq i}^{n} u_{1 j} K\left(\frac{x_{i} \zeta_{0}-x_{j} \zeta_{0}}{a_{n}}\right) .
\end{aligned}
$$

Let $A_{i}=\mathrm{E}\left(x_{(2) i} \mid x_{i} \zeta_{0}\right) p\left(x_{i} \zeta_{0} \mid \delta_{0}\right), \quad B_{i}=p\left(x_{i} \zeta_{0} \mid \delta_{0}\right)$, and $C_{i}=\mathrm{E}\left(u_{1 i} \mid x_{i} \zeta_{0}\right) \times$ $p\left(x_{i} \zeta_{0} \mid \delta_{0}\right)$ be their limits. By a Taylor expansion,

where

$$
\begin{aligned}
C_{0, n}= & \frac{1}{\sqrt{n}} \sum_{i=1}^{n} t_{n}\left(x_{i} \zeta_{0}, \xi_{0}\right)\left(x_{(2) i} \quad \mathrm{E}\left(x_{(2) i} \mid x_{i} \zeta_{0}\right)\right)^{\prime}\left(u_{1 i} \quad \mathrm{E}\left(u_{1 i} \mid x_{i} \zeta_{0}\right)\right) \\
& +L_{n}+R_{n},
\end{aligned}
$$

$$
\begin{aligned}
L_{n}= & -\frac{1}{\sqrt{n}} \sum_{i=1}^{n} t_{n}\left(x_{i} \zeta_{0}, \zeta_{0}\right) \frac{1}{B_{i}}\left(u_{1 i}-\mathrm{E}\left(u_{1 i} \mid x_{i} \zeta_{0}\right)\right)\left(A_{n i}-A_{i}\right)^{\prime} \\
& -\frac{1}{\sqrt{n}} \sum_{i=1}^{n} t_{n}\left(x_{i} \zeta_{0}, \zeta_{0}\right)\left(x_{(2) i}-\mathrm{E}\left(x_{(2) i} \mid x_{i} \zeta_{0}\right)\right)^{\prime} \frac{1}{B_{i}}\left(C_{n i}-C_{i}\right) \\
& +\frac{1}{\sqrt{n}} \sum_{i=1}^{n} t_{n}\left(x_{i} \zeta_{0}, \zeta_{0}\right)\left[\mathrm{E}\left(x_{(2) i} \mid x_{i} \zeta_{0}\right)^{\prime}\left(u_{1 i}-\mathrm{E}\left(u_{1 i} \mid x_{i} \zeta_{0}\right)\right)\right. \\
& \left.+\left(x_{(2) i}-\mathrm{E}\left(x_{(2) i} \mid x_{i} \zeta_{0}\right)\right)^{\prime} \mathrm{E}\left(u_{1 i} \mid x_{i} \zeta_{0}\right)\right] \frac{1}{B_{i}}\left(B_{n i}-B_{i}\right)
\end{aligned}
$$

and

$$
\begin{aligned}
R_{n}= & \frac{2}{\sqrt{n}} \sum_{i=1}^{n} t_{n}\left(x_{i} \zeta_{0}, \xi_{0}\right) \frac{1}{\tilde{B}_{n i}^{2}}\left(A_{n i}-A_{i}\right)^{\prime}\left(C_{n i}-C_{i}\right) \\
& +\frac{2}{\sqrt{n}} \sum_{i=1}^{n} t_{n}\left(x_{i} \zeta_{0}, \xi_{0}\right)\left[\left(u_{1 i} \tilde{B}_{n i}-\tilde{C}_{n i}\right)-\tilde{C}_{n i}\right] \frac{1}{\tilde{B}_{n i}^{3}}\left(A_{n i}-A_{i}\right)^{\prime}\left(B_{n i}-B_{i}\right) \\
& +\frac{2}{\sqrt{n}} \sum_{i=1}^{n} t_{n}\left(x_{i} \zeta_{0}, \xi_{0}\right)\left[-\tilde{A}_{n i}^{\prime}+\left(x_{2 i} \tilde{B}_{n i}-\tilde{A}_{n i}\right)^{\prime}\right] \frac{1}{\tilde{B}_{n i}^{3}}\left(B_{n i}-B_{i}\right)\left(C_{n i}-C_{i}\right) \\
& +\frac{2}{\sqrt{n}} \sum_{i=1}^{n} t_{n}\left(x_{i} \zeta_{0}, \xi_{0}\right)\left[-\tilde{A}_{n i}^{\prime}\left(u_{1 i} \tilde{B}_{n i}-\tilde{C}_{n i}\right)+\tilde{A}_{n i}^{\prime} \tilde{C}_{n i}-\left(x_{2 i} \tilde{B}_{n i}-\tilde{A}_{n i}\right)^{\prime} \tilde{C}_{n i}\right] \\
& \times \frac{1}{\tilde{B}_{n i}^{4}}\left(B_{n i}-B_{i}\right)^{2} .
\end{aligned}
$$


Since $\lim _{n \rightarrow \infty} n a_{n}^{2 m}=\infty$ and $\lim _{n \rightarrow \infty} n a_{n}^{4 s^{*}}=0, R_{n}$ converges to zero in probability by Proposition 3. As $\lim _{n \rightarrow \infty} n a_{n}^{m}=\infty, L_{n}$ converges to zero in probability by Proposition 4. Hence

$$
\begin{aligned}
\frac{1}{\sqrt{n}} \hat{X}_{2}^{\prime} \hat{U}_{n}= & \frac{1}{\sqrt{n}} \sum_{i=1}^{n} t_{n}\left(x_{i} \zeta_{0}, \xi_{0}\right)\left(x_{(2) i}-\mathrm{E}\left(x_{(2) i} \mid x_{i} \zeta_{0}\right)\right)^{\prime}\left(u_{1 i}-\mathrm{E}\left(u_{1 i} \mid x_{i} \zeta_{0}\right)\right) \\
& -\mathrm{E}\left(I_{T}\left(x \zeta_{0}\right)\left(x_{(2)}-\mathrm{E}\left(x_{(2)} \mid x \zeta_{0}\right)\right)^{\prime} \frac{\partial \mathrm{E}\left(u_{1} \mid x \zeta_{0}, \delta_{0}\right)}{\partial \delta^{\prime}}\right) \\
& \times \sqrt{n}\left(\tilde{\delta}-\delta_{0}\right)+o_{p}(1)
\end{aligned}
$$

which is asymptotically normal $\mathrm{N}(0, A)$, where $\Delta$ is defined in (4.11), under the property that $\sqrt{n}\left(\hat{\delta}-\delta_{0}\right)$ is asymptotically uncorrelated with $\left(u_{1}-\mathrm{E}\left(u_{1} \mid x \zeta_{0}\right)\right)$.

For the asymptotic distribution of the SG2LS, by a mcan valuc theorem

$$
\begin{aligned}
& \frac{1}{\sqrt{n}} \hat{X}_{2}^{\prime} \hat{A}_{n}^{-1} \hat{U}_{n} \\
& =\frac{1}{\sqrt{n}} \sum_{i=1}^{n} t_{n}\left(x_{i}, \hat{\zeta}, \hat{\xi}_{n}\right)\left(x_{(2) t}-\mathbf{E}_{n}\left(x_{(2)} \mid x_{i} \hat{\zeta}\right)\right)^{\prime} \omega_{n i}^{-1}(\hat{\beta}, \hat{\delta})\left(u_{1 i}-\mathbf{E}_{n}\left(u_{1} \mid x_{i} \hat{\zeta}\right)\right) \\
& =\frac{1}{\sqrt{n}} \sum_{i=1}^{n} t_{n}\left(x_{i} \zeta_{0}, \zeta_{0}\right)\left(x_{(2) i}-\mathrm{E}_{n}\left(x_{(2)} \mid x_{i} \zeta_{0}\right)\right)^{\prime} \omega_{n i}^{-1}\left(\beta_{0}, \delta_{0}\right)\left(u_{1 i}-\mathrm{E}_{n}\left(u_{1} \mid x_{i} \zeta_{0}\right)\right) \\
& -\frac{1}{n} \sum_{i=1}^{n} t_{n}\left(x_{i} \bar{\zeta}, \bar{\xi}\right)\left(x_{(2) i}-\mathrm{E}_{n}\left(x_{(2)} \mid x_{i} \bar{\zeta}\right)\right)^{\prime} \omega_{n i}^{-1}(\bar{\beta}, \bar{\delta}) \frac{\partial \mathrm{E}_{n}\left(u_{1} \mid x_{i} \bar{\zeta}\right)}{\partial \delta^{\prime}} \sqrt{n}\left(\hat{\delta}-\delta_{0}\right) \\
& +\frac{1}{n} \sum_{i=1}^{n}\left(u_{1 i}-\mathrm{E}_{n}\left(u_{1} \mid x_{i} \bar{\zeta}\right)\right) \frac{\partial}{\partial \delta^{\prime}}\left[t_{n}\left(x_{i} \bar{\zeta}, \bar{\zeta}\right)\left(x_{(2) i}-\mathrm{E}_{n}\left(x_{(2)} \mid x_{i} \bar{\zeta}\right)\right)^{\prime} \omega_{n i}^{-1}(\bar{\beta}, \bar{\delta})\right] \\
& \times \sqrt{n}\left(\hat{\delta}-\delta_{0}\right)+\frac{1}{n} \sum_{i=1}^{n} t_{n}\left(x_{i} \zeta, \xi\right)\left(u_{1 i}-\mathrm{E}_{n}\left(u_{1} \mid x_{i} \bar{\zeta}\right)\right)\left(x_{(2) i}-\mathrm{E}_{n}\left(x_{(2)} \mid x_{i} \bar{\zeta}\right)\right)^{\prime} \\
& \times \frac{\partial \omega_{n i}^{-1}(\bar{\beta}, \bar{\delta})}{\partial \beta^{\prime}} \sqrt{n}\left(\hat{\beta}-\beta_{0}\right)+\frac{1}{n} \sum_{i=1}^{n}\left(u_{1 i}-\mathrm{E}_{n}\left(u_{1} \mid x_{i} \bar{\zeta}\right)\right)\left(x_{(2) i}-\mathrm{E}_{n}\left(x_{(2)} \mid x_{i} \bar{\zeta}\right)\right)^{\prime} \\
& \times \omega_{n i}^{-1}(\bar{\beta}, \bar{\delta}) \frac{\partial t_{n}\left(x_{i} \bar{\zeta}, \bar{\xi}\right)}{\partial \xi^{\prime}} \sqrt{n}\left(\hat{\xi}_{n}-\xi_{0}\right) \\
& =C_{0, n}^{(\omega)}-\mathrm{E}\left[I_{T}\left(x \zeta_{0}\right)\left(x_{(2)}-\mathrm{E}\left(x_{(2)} \mid x \zeta_{0}\right)\right)^{\prime} \omega^{-1}\left(x \zeta_{0}, \delta_{0}\right) \frac{\mathrm{\partial E}\left(u_{1} \mid x \zeta_{0}, \delta_{0}\right)}{\partial \delta^{\prime}}\right] \\
& \times \sqrt{n}\left(\hat{\delta}-\delta_{0}\right)+\mathrm{o}_{p}(1)
\end{aligned}
$$


where

$$
\begin{aligned}
C_{0, n}^{(\omega)}= & \frac{1}{\sqrt{n}} \sum_{i=1}^{n} t_{n}\left(x_{i} \zeta_{0}, \zeta_{0}\right)\left(x_{(2) i}-\mathrm{E}_{n}\left(x_{(2)} \mid x_{i} \zeta_{0}\right)\right)^{\prime} \\
& \times \omega_{n i}^{-1}\left(\beta_{0}, \delta_{0}\right)\left(u_{1 i}-\mathrm{E}_{n}\left(u_{1} \mid x_{i} \zeta_{0}\right)\right) .
\end{aligned}
$$

To simplify notations, let

$$
\begin{aligned}
& H_{n i}=\frac{1}{(n-1) b_{n}^{m}} \sum_{j \neq i}^{n} H\left(\frac{x_{i} \zeta_{0}-x_{j} \zeta_{0}}{b_{n}}\right), \\
& R_{n i}=\frac{1}{(n-1) b_{n}^{m}} \sum_{j \neq i}^{n} u_{1 j} H\left(\frac{x_{i} \zeta_{0}-x_{j} \zeta_{0}}{b_{n}}\right), \\
& S_{n i}=\frac{1}{(n-1) b_{n}^{m}} \sum_{j \neq i}^{n} u_{1 j}^{2} H\left(\frac{x_{i} \zeta_{0}-x_{j} \zeta_{0}}{b_{n}}\right) .
\end{aligned}
$$

Let $H_{i}=B_{i}, R_{i}=C_{i}$, and $S_{i}=\mathrm{E}\left(u_{1 i}^{2} \mid x_{i} \zeta_{0}\right) p\left(x_{i} \zeta_{0} \mid \delta_{0}\right)$ be their limits respectively. By Proposition 3, as $\lim _{n \rightarrow \infty} n a_{n}^{2 m}=\infty, \lim _{n \rightarrow \infty} n a_{n}^{4 s^{*}}-0, \lim _{n \rightarrow \infty} n b_{n}^{2 m}=\infty$, and $\lim _{n \rightarrow \infty} n b_{n}^{4 h^{*}}=0$,

$$
\begin{aligned}
C_{0, n}^{(\omega)}= & \frac{1}{\sqrt{n}} \sum_{i=1}^{n} t_{n}\left(x_{i} \zeta_{0}, \xi_{0}\right)\left(x_{(2) i}-\mathrm{E}\left(x_{(2) i} \mid x_{i} \zeta_{0}\right)\right)^{\prime} \\
& \times \omega^{-1}\left(x_{i} \zeta_{0}, \delta_{0}\right)\left(u_{1 i}-\mathrm{E}\left(u_{1 i} \mid x_{i} \zeta_{0}\right)\right)+L_{n}^{(\omega)}+o_{p}(1),
\end{aligned}
$$

where

$$
\begin{aligned}
L_{n}^{(w)}= & -\frac{1}{\sqrt{n}} \sum_{i=1}^{n} t_{n}\left(x_{i} \zeta_{0}, \zeta_{0}\right) \omega^{-1}\left(x_{i} \zeta_{0}, \delta_{0}\right)\left(u_{1 i}-\mathrm{E}\left(u_{1 i} \mid x_{i} \zeta_{0}\right)\right) \frac{1}{B_{i}}\left(A_{n i}-A_{i}\right)^{\prime} \\
& -\frac{1}{\sqrt{n}} \sum_{i=1}^{n} t_{n}\left(x_{i} \zeta_{0}, \zeta_{0}\right) \omega^{-1}\left(x_{i} \zeta_{0}, \delta_{0}\right)\left(x_{(2) i}-\mathrm{E}\left(x_{(2) i} \mid x_{i} \zeta_{0}\right)\right)^{\prime} \frac{1}{B_{i}}\left(C_{n i}-C_{i}\right) \\
& +\frac{1}{\sqrt{n}} \sum_{i=1}^{n} t_{n}\left(x_{i} \zeta_{0}, \zeta_{0}\right) \omega^{-1}\left(x_{i} \zeta_{0}, \delta_{0}\right)\left[\mathrm{E}\left(x_{(2) i} \mid x_{i} \zeta_{0}\right)^{\prime}\left(u_{1 i}-\mathrm{E}\left(u_{1 i} \mid x_{i} \zeta_{0}\right)\right)\right. \\
& \left.+\left(x_{(2) i}-\mathrm{E}\left(x_{(2) i} \mid x_{i} \zeta_{0}\right)\right)^{\prime} \mathrm{E}\left(u_{1 i} \mid x_{i} \zeta_{0}\right)\right] \frac{1}{B_{i}}\left(B_{n i}-B_{i}\right) \\
& -\frac{1}{\sqrt{n}} \sum_{i=1}^{n} t_{n}\left(x_{i} \zeta_{0}, \xi_{0}\right) \omega^{-2}\left(x_{i} \zeta_{0}, \delta_{0}\right)\left(x_{(2) i}-\mathrm{E}\left(x_{(2) i} \mid x_{i} \zeta_{0}\right)\right)^{\prime} \\
& \times\left(u_{1 i}-\mathrm{E}\left(u_{1 i} \mid x_{i} \zeta_{0}\right)\right) \frac{1}{H_{i}}\left\{\left(S_{n i}-S_{i}\right)-2 \mathrm{E}\left(u_{1 i} \mid x_{i} \zeta_{0}\right)\left(R_{n i}-R_{i}\right)\right. \\
& \left.-\left[\mathrm{E}\left(u_{1 i}^{2} \mid x_{i} \zeta_{0}\right)-2 \mathrm{E}\left(u_{1 i} \mid x_{i} \zeta_{0}\right)\right]\left(H_{n i}-H_{i}\right)\right\} .
\end{aligned}
$$


Since $\mathrm{E}\left[x_{(2) i}-\mathrm{E}\left(x_{(2) i} \mid x_{i} \zeta_{0}\right) \mid x_{i} \zeta_{0}\right]=0, \mathrm{E}\left[u_{1 i}-\mathrm{E}\left(u_{1 i} \mid x_{i} \zeta_{0}\right) \mid x_{i}\right]=0$, and the other functions in $L_{n}^{(\omega)}$ are functions of $x_{i} \zeta_{0}$, Proposition 4 implies that $L_{n}^{(\omega)}$ converges to zero in probability when $\lim _{n \rightarrow \infty} n a_{n}^{m}=\infty$ and $\lim _{n \rightarrow \infty} n b_{n}^{m}=\infty$. Similarly,

$$
\begin{aligned}
& \frac{1}{\sqrt{n}} \frac{\partial \mathrm{E}_{n}(\hat{\beta}, \hat{\zeta})}{\partial \delta} \hat{\Lambda}_{n}^{-1} \hat{U}_{n} \\
& =\frac{1}{\sqrt{n}} \sum_{i=1}^{n} t_{n}\left(x_{i} \hat{\zeta}, \hat{\xi}_{n}\right) \frac{\partial \mathrm{E}_{n}\left(y-w \hat{\beta} \mid x_{i} \hat{\zeta}\right)}{\partial \delta} \omega_{n i}^{-1}(\hat{\beta}, \hat{\delta})\left(u_{1 i}-\mathrm{E}_{n}\left(u_{1} \mid x_{i} \hat{\zeta}\right)\right) \\
& =\frac{1}{\sqrt{n}} \sum_{i=1}^{n} t_{n}\left(x_{i} \zeta_{0}, \xi_{0}\right) \frac{\partial \mathrm{E}_{n}\left(u_{1} \mid x_{i} \zeta_{0}\right)}{\partial \delta} \omega_{n i}^{-1}\left(\beta_{0}, \dot{\delta}_{0}\right)\left(u_{1 i}-\mathrm{E}_{n}\left(u_{1} \mid x_{i} \zeta_{0}\right)\right) \\
& -\frac{1}{n} \sum_{i=1}^{n} t_{n}\left(x_{i} \bar{\zeta}, \bar{\xi}\right) \frac{\partial \mathrm{E}_{n}\left(y-w \bar{\beta} \mid x_{i} \bar{\zeta}\right)}{\partial \delta} \omega_{n i}^{-1}(\bar{\beta}, \bar{\delta}) \frac{\partial \mathrm{E}_{n}\left(u_{1} \mid x_{i} \bar{\zeta}\right)}{\partial \delta^{\prime}} \sqrt{n}\left(\hat{\delta}-\delta_{0}\right) \\
& +\frac{1}{n} \sum_{i=1}^{n}\left(u_{1 i}-\mathrm{E}_{n}\left(u_{1} \mid x_{i} \bar{\zeta}\right)\right) \frac{\partial}{\partial \delta^{\prime}}\left[t\left(x_{i} \bar{\zeta}, \bar{\xi}\right) \frac{\partial \mathrm{E}_{n}\left(y \quad w \bar{\beta} \mid x_{i} \bar{\zeta}\right)}{\partial \delta} \omega_{n i}^{-1}(\bar{\beta}, \bar{\delta})\right] \\
& \times \sqrt{n}\left(\bar{\delta}-\delta_{0}\right)+\frac{1}{n} \sum_{i=1}^{n} t_{n}\left(x_{i} \zeta, \xi\right)\left(u_{1 i}-\mathrm{E}_{n}\left(u_{1} \mid x_{i} \zeta\right)\right) \\
& \times \frac{\partial}{\partial \beta^{\prime}}\left[\frac{\partial \mathrm{E}_{n}\left(y-w \bar{\beta} \mid x_{i} \bar{\zeta}\right)}{\partial \delta} \omega_{n i}^{-1}(\bar{\beta}, \bar{\delta})\right] \sqrt{n}\left(\hat{\beta}-\beta_{0}\right) \\
& +\frac{1}{n} \sum_{i=1}^{n}\left(u_{1 i}-\mathrm{E}_{n}\left(u_{1} \mid x_{i} \bar{\zeta}\right)\right) \frac{\partial \mathrm{E}_{n}\left(y-w \bar{\beta} \mid x_{i} \bar{\zeta}\right)}{\partial \delta} \\
& \times \omega_{n i}^{-1}(\bar{\beta}, \bar{\delta}) \frac{\partial t\left(x_{i} \bar{\zeta}, \bar{\xi}\right)}{\partial \delta^{\prime}} \sqrt{n}\left(\hat{\xi}_{n}-\xi_{0}\right) \\
& =C_{0, n}^{(d)}-\mathrm{E}\left[I_{T}\left(x \zeta_{0}\right) \frac{\partial \mathrm{E}\left(u_{1} \mid x \zeta_{0}, \delta_{0}\right)}{\partial \delta} \omega^{-1}\left(x \zeta_{0}, \delta_{0}\right) \frac{\partial \mathrm{E}\left(u_{1} \mid x \zeta_{0}, \delta_{0}\right)}{\partial \delta^{\prime}}\right] \\
& \times \sqrt{n}\left(\hat{\delta}-\delta_{0}\right)+o_{p}(1),
\end{aligned}
$$

where

$$
C_{0, n}^{(d)}=\frac{1}{\sqrt{n}} \sum_{i=1}^{n} t_{n}\left(x_{i} \zeta_{0}, \zeta_{0}\right) \frac{\partial \mathrm{E}_{n}\left(u_{1} \mid x_{i} \zeta_{0}, \delta_{0}\right)}{\partial \delta} \omega_{n}^{-1}\left(\beta_{0}, \delta_{0}\right)\left(u_{1 i}-\mathrm{E}_{n}\left(u_{1} \mid x_{i} \zeta_{0}\right)\right) .
$$

Since $\lim _{n \rightarrow \infty} n a_{n}^{2(m+2)}=\infty, \quad \lim _{n \rightarrow \infty} n a_{n}^{4 s^{*}}=0, \quad \lim _{n \rightarrow \infty} n b_{n}^{2 m}=\infty, \quad$ and $\lim _{n \rightarrow \infty} n b_{n}^{4 h^{*}}=0$, Proposition 3 implies that

$$
\begin{aligned}
C_{0, n}^{(d)}= & \frac{1}{\sqrt{n}} \sum_{i=1}^{n} t_{n}\left(x_{i} \zeta_{0}, \zeta_{0}\right) \frac{\partial \mathrm{E}\left(u_{1} \mid x_{i} \zeta_{0}, \delta_{0}\right)}{\partial \delta} \omega^{-1}\left(x_{i} \zeta_{0}, \delta_{0}\right)\left(u_{1 i}-\mathrm{E}\left(u_{1 i} \mid x_{i} \zeta_{0}\right)\right) \\
& +I_{0, n}^{(d)}+\sigma_{p}(1),
\end{aligned}
$$


where

$$
\begin{aligned}
& L_{0, n}^{(d)}=-\frac{1}{\sqrt{n}} \sum_{i=1}^{n} t_{n}\left(x_{i} \zeta_{0}, \xi_{0}\right) \frac{\partial \mathrm{E}\left(u_{1} \mid x_{i} \zeta_{0}, \delta_{0}\right)}{\partial \delta} \omega^{-1}\left(x_{i} \zeta_{0}, \delta_{0}\right) \frac{1}{B_{i}}\left(A_{n i}-A_{i}\right) \\
& +\frac{1}{\sqrt{n}} \sum_{i=1}^{n} t_{n}\left(x_{i} \zeta_{0}, \xi_{0}\right) \omega^{-1}\left(x_{i} \zeta_{0}, \delta_{0}\right)\left[\frac{\partial \mathrm{E}\left(u_{1} \mid x_{i} \zeta_{0}, \delta_{0}\right)}{\partial \delta} A_{i}\right. \\
& \left.-\left(u_{1 i}-\mathrm{E}\left(u_{1 i} \mid x_{i} \zeta_{0}\right)\right)\left\{\frac{\partial C_{i}}{\partial \delta}-2 \mathrm{E}\left(u_{1 i} \mid x_{i} \zeta_{0}\right) \frac{\partial B_{i}}{\partial \delta}\right\}\right] \frac{1}{B_{i}^{2}}\left(B_{n i}-B_{i}\right) \\
& -\frac{1}{\sqrt{n}} \sum_{i=1}^{n} t_{n}\left(x_{i} \zeta_{0}, \xi_{0}\right) \omega^{-1}\left(x_{i} \zeta_{0}, \delta_{0}\right)\left(u_{1 i}-\mathrm{E}\left(u_{1 i} \mid x_{i} \zeta_{0}\right)\right) \\
& \times \frac{1}{B_{i}^{2}} \frac{\partial B_{i}}{\partial \delta}\left(C_{n i}-C_{i}\right)-\frac{1}{\sqrt{n}} \sum_{i=1}^{n} t_{n}\left(x_{i} \zeta_{0}, \xi_{0}\right) \omega^{-1}\left(x_{i} \zeta_{0}, \delta_{0}\right) \\
& \times\left(u_{1 i}-\mathrm{E}\left(u_{1 i} \mid x_{i} \zeta_{0}\right)\right) \mathrm{E}\left(u_{1 i} \mid x_{i} \zeta_{0}\right) \frac{1}{B_{i}}\left(\frac{\partial B_{n i}}{\partial \delta}-\frac{\partial B_{i}}{\partial \delta}\right) \\
& +\frac{1}{\sqrt{n}} \sum_{i=1}^{n} t_{n}\left(x_{i} \zeta_{0}, \xi_{0}\right) \omega^{-1}\left(x_{i} \zeta_{0}, \delta_{0}\right)\left(u_{1 i}-\mathrm{E}\left(u_{1 i} \mid x_{i} \zeta_{0}\right)\right) \frac{1}{B_{i}}\left(\frac{\partial C_{n i}}{\partial \delta}-\frac{\partial C_{i}}{\partial \delta}\right) \\
& +\frac{2}{\sqrt{n}} \sum_{i=1}^{n} t_{n}\left(x_{i} \zeta_{0}, \xi_{0}\right) \omega^{-2}\left(x_{i} \zeta_{0}, \delta_{0}\right)\left(u_{1 i}-\mathrm{E}\left(u_{1 i} \mid x_{i} \zeta_{0}\right)\right) \frac{\partial \mathrm{E}\left(u_{1} \mid x_{i} \zeta_{0}, \delta_{0}\right)}{\partial \delta} \\
& \times \mathrm{E}\left(u_{1 i} \mid x_{i} \zeta_{0}\right) \frac{1}{H_{i}}\left(R_{n i}-R_{i}\right)-\frac{1}{\sqrt{n}} \sum_{i=1}^{n} t_{n}\left(x_{i} \zeta_{0}, \zeta_{0}\right) \frac{\partial \mathrm{E}\left(u_{1} \mid x_{i} \zeta_{0}, \delta_{0}\right)}{\partial \delta} \\
& \times \omega^{-2}\left(x_{i} \zeta_{0}, \delta_{0}\right)\left(u_{1 i}-\mathrm{E}\left(u_{1 i} \mid x_{i} \zeta_{0}\right)\right) \frac{1}{H_{i}}\left(S_{n i}-S_{i}\right) \\
& +\frac{1}{\sqrt{n}} \sum_{i=1}^{n} t_{n}\left(x_{i} \zeta_{0}, \xi_{0}\right) \omega^{-2}\left(x_{i} \zeta_{0}, \delta_{0}\right)\left(u_{1 i}-\mathrm{E}\left(u_{1 i} \mid x_{i} \zeta_{0}\right)\right) \frac{\partial \mathrm{E}\left(u_{1} \mid x_{i} \zeta_{0}, \delta_{0}\right)}{\partial \delta} \\
& \times\left(S_{i}-2 \frac{R_{i}^{2}}{H_{i}}\right) \frac{1}{H_{i}^{2}}\left(H_{n i}-H_{i}\right) \text {. }
\end{aligned}
$$

Since

$$
\frac{\partial \mathrm{E}\left(u_{1} \mid x \zeta_{0}, \delta_{0}\right)}{\partial \delta_{k}}=\left(x-\mathrm{E}\left(x \mid x \zeta_{0}\right)\right)^{\prime} \frac{\partial \zeta\left(\delta_{0}\right)}{\partial \delta_{k}} \nabla \mathrm{E}\left(u_{1} \mid x \zeta_{0}\right)
$$

by Propositions 4 and $6, L_{0, n}^{(d)}$ converges to zero in probability as $\lim _{n \rightarrow \infty} n a_{n}^{m+2}=\infty$ and $\lim _{n \rightarrow \infty} n b_{n}^{m}=\infty$. Hence it follows after some matrix 
manipulation and simplification that

$$
\begin{aligned}
& \frac{1}{\sqrt{n}} \hat{X}_{2}^{\prime} \hat{\Sigma}^{-1} \hat{U}_{n} \\
&=\frac{1}{\sqrt{n}} \hat{X}_{2}^{\prime} \hat{A}_{n}^{-1} \hat{U}_{n}-\hat{X}_{2}^{\prime} \hat{A}_{n}^{-1} \frac{\partial \mathrm{E}_{n}(\hat{\beta}, \hat{\zeta})}{\partial \delta^{\prime}}\left(\hat{V}_{n, \delta}^{-1}+\frac{\partial \mathrm{E}_{n}^{\prime}(\hat{\beta}, \bar{\zeta})}{\partial \delta} \hat{\Lambda}_{n}^{-1} \frac{\partial \mathrm{E}_{n}(\hat{\beta}, \hat{\zeta})}{\partial \delta^{\prime}}\right)^{-1} \\
& \quad \times \frac{1}{\sqrt{n}} \frac{\partial \mathrm{E}_{n}^{\prime}(\hat{\beta}, \hat{\zeta})}{\partial \delta} \hat{\Lambda}_{n}^{-1} \hat{U}_{n} \\
&=\frac{1}{\sqrt{n}} \sum_{i=1}^{n} t_{n}\left(x_{i} \zeta_{0}, \zeta_{0}\right)\left(x_{(2) i}-\mathrm{E}\left(x_{(2) i} \mid x_{i} \zeta_{0}\right)\right)^{\prime} \omega^{-1}\left(x_{i} \zeta_{0}, \delta_{0}\right)\left(u_{1 i}-\mathrm{E}\left(u_{1 i} \mid x_{i} \zeta_{0}\right)\right) \\
&-\mathrm{E}\left[I_{T}\left(x \zeta_{0}\right)\left(x_{(2)}-\mathrm{E}\left(x_{(2)} \mid x \zeta_{0}\right)\right)^{\prime} \omega^{-1}\left(x \zeta_{0}, \delta_{0}\right) \frac{\partial \mathrm{E}\left(u_{1} \mid x \zeta_{0}, \delta_{0}\right)}{\partial \delta^{\prime}}\right] \\
& \times\left(V_{\delta}^{-1}+\mathrm{E}\left[I_{T}\left(x \zeta_{0}\right) \frac{\partial \mathrm{E}\left(u_{1} \mid x \zeta_{0}, \delta_{0}\right)}{\partial \delta^{\prime}} \omega^{-1}\left(x \zeta_{0}, \delta_{0}\right) \frac{\partial \mathrm{E}\left(u_{1} \mid x \zeta_{0}, \delta_{0}\right)}{\partial \delta^{\prime}}\right]\right)^{-1} \\
& \times\left\{\frac{1}{\sqrt{n}} \sum_{i=1}^{n} t_{n}\left(x_{i} \zeta_{0}, \zeta_{0}\right) \frac{\partial \mathrm{E}\left(u_{1} \mid x_{i} \zeta_{0}, \dot{\delta}_{0}\right)}{\partial \delta} \omega^{-1}\left(x_{i} \zeta_{0}, \delta_{0}\right)\left(u_{l i}-\mathrm{E}\left(u_{1 i} \mid x_{i} \zeta_{0}\right)\right)\right. \\
&\left.+V V_{\delta}^{-1} \sqrt{n}\left(\hat{\delta}-\delta_{0}\right)\right\}+\mathrm{o}_{p}(1) \\
& \stackrel{D}{\rightarrow} \mathrm{N}(0, \Gamma),
\end{aligned}
$$

where $\Gamma$ is in (5.12).

For the instrumental variable estimator in (5.18),

$$
\begin{aligned}
\frac{1}{\sqrt{n}} P^{\prime} \hat{U}_{n}= & \frac{1}{\sqrt{n}} \sum_{i=1}^{n} t_{n}\left(x_{i} \zeta_{0}, \xi_{0}\right) p_{i}^{\prime}\left(u_{1 i}-\mathrm{E}_{n}\left(u_{1} \mid x_{i} \zeta_{0}\right)\right) \\
& -\frac{1}{n} \sum_{i=1}^{n} t_{n}\left(x_{i} \bar{\zeta}, \bar{\xi}\right) p_{i}^{\prime} \frac{\partial \mathrm{E}_{n}\left(u_{1} \mid x_{i} \bar{\zeta}\right)}{\partial \delta^{\prime}} \sqrt{n}\left(\hat{\delta}-\delta_{0}\right) \\
& +\frac{1}{n} \sum_{i=1}^{n} p_{i}^{\prime}\left(u_{1 i}-\mathrm{E}_{n}\left(u_{1} \mid x_{i} \bar{\zeta}\right)\right) \frac{\partial t_{n}\left(x_{i} \bar{\zeta}, \bar{\xi}\right)}{\partial \delta^{\prime}} \sqrt{n}\left(\hat{\delta}-\delta_{0}\right) \\
& +\frac{1}{n} \sum_{i=1}^{n} p_{i}^{\prime}\left(u_{1 i}-\mathrm{E}_{n}\left(u_{1} \mid x_{i} \bar{\zeta}\right)\right) \frac{\partial t_{n}\left(x_{i} \bar{\zeta}, \bar{\xi}\right)}{\partial \bar{\xi}^{\prime}} \sqrt{n}\left(\hat{\xi} n-\xi_{0}\right) \\
= & \frac{1}{\sqrt{n}} \sum_{i=1}^{n} t_{n}\left(x_{i} \zeta_{0}, \xi_{0}\right) p_{i}^{\prime}\left(u_{1 i}-\mathrm{E}_{n}\left(u_{1} \mid x_{i} \zeta_{0}\right)\right) \\
& -\mathrm{E}\left[I_{T}\left(x_{\zeta_{0}}\right) p^{\prime} \frac{\partial \mathrm{E}\left(u_{1} \mid x \zeta_{0}, \delta_{0}\right)}{\partial \delta^{\prime}}\right] \sqrt{n}\left(\hat{\delta}-\delta_{0}\right)+o_{p}(1) .
\end{aligned}
$$




\section{Since}

$$
\frac{\partial \mathrm{E}\left(u_{1} \mid x \zeta_{0}, \delta_{0}\right)}{\partial \delta_{k}}=\left(x-\mathrm{E}\left(x \mid x \zeta_{0}\right)\right)^{\prime} \frac{\partial \zeta\left(\delta_{0}\right)}{\partial \delta_{k}} \nabla \mathrm{E}\left(u_{1} \mid x \zeta_{0}\right),
$$

it follows that

$$
\mathrm{E}\left[\mathrm{E}\left(p^{\prime} \mid x \zeta_{0}\right) \frac{\partial \mathrm{E}\left(u_{1} \mid x \zeta_{0}, \delta_{0}\right)}{\partial \delta^{\prime}}\right]=0,
$$

and therefore,

$$
\mathrm{E}\left[I_{T}\left(x \zeta_{0}\right) p^{\prime} \frac{\partial \mathrm{E}\left(u_{1} \mid x \zeta_{0}, \delta_{0}\right)}{\partial \delta^{\prime}}\right]=\mathrm{E}\left[I_{T}\left(x \zeta_{0}\right)\left(p-\mathrm{E}\left(p \mid x \zeta_{0}\right)\right)^{\prime} \frac{\partial \mathrm{E}\left(u_{1} \mid x \zeta_{0}, \delta_{0}\right)}{\partial \delta^{\prime}}\right] .
$$

On the other hand,

$$
\begin{aligned}
& \frac{1}{\sqrt{n}} \sum_{i=1}^{n} t_{n}\left(x_{i} \zeta_{0}, \xi_{0}\right) p_{i}^{\prime}\left(u_{1 i}-\mathrm{E}_{n}\left(u_{1} \mid x_{i} \zeta_{0}\right)\right) \\
& =\frac{1}{\sqrt{n}} \sum_{i=1}^{n} t_{n}\left(x_{i} \zeta_{0}, \zeta_{0}\right) p_{i}^{\prime}\left(u_{1 i}-\mathrm{E}\left(u_{1 i} \mid x_{i} \zeta_{0}\right)\right) \\
& \quad-\frac{1}{\sqrt{n}} \sum_{i=1}^{n} t_{n}\left(x_{i} \zeta_{0}, \xi_{0}\right) p_{i}^{\prime} \frac{1}{B_{i}}\left(C_{n i}-C_{i}\right) \\
& \quad+\frac{1}{\sqrt{n}} \sum_{i=1}^{n} t_{n}\left(x_{i} \zeta_{0}, \zeta_{0}\right) p_{i}^{\prime} \mathrm{E}\left(u_{1 i} \mid x_{i} \zeta_{0}\right) \frac{1}{B_{i}}\left(B_{n i}-B_{i}\right)+\mathrm{o}_{p}(1) .
\end{aligned}
$$

With a high-order kernel of orders $s^{*}$ such that $\lim _{n \rightarrow \infty} a_{n}^{2 s^{*}}=0,{ }^{11}$ Proposition 5 implies

$$
\begin{aligned}
& \frac{1}{\sqrt{n}} \sum_{i=1}^{n} t_{n}\left(x_{i} \zeta_{0}, \xi_{0}\right) p_{i}^{\prime} \frac{1}{B_{i}}\left(C_{n i}-C_{i}\right) \\
& =\frac{1}{\sqrt{n}} \sum_{i=1}^{n}\left\{I_{T}\left(x_{i} \zeta_{0}\right) u_{1 i} \mathrm{E}\left(p_{i}^{\prime} \mid x_{i} \zeta_{0}\right)-\mathrm{E}\left[I_{T}\left(x_{i} \zeta_{0}\right) \mathrm{E}\left(u_{1 i} \mid x_{i} \zeta_{0}\right) \mathrm{E}\left(p_{i}^{\prime} \mid x_{i} \zeta_{0}\right)\right]\right\}+o_{p}(1)
\end{aligned}
$$

\footnotetext{
${ }^{11}$ This rate requirement implies $\lim _{n \rightarrow \infty} n a_{n}^{4 s^{*}}=0$ in Assumption 2. This stronger requirement is nceded only here. It guarantees that the asymptotic bias of the following term will converge to zero. Using $p_{i}-\mathbf{E}_{n}\left(p \mid x_{i} \zeta\right)$ instead of $p_{i}$ will eliminate such an asymptotic bias and this stronger rate requirement will not be needed. This indicates the advantage of using $p_{i}-\mathrm{E}_{n}\left(p \mid x_{i} \bar{\zeta}\right)$ as an instrumental variable instead of $p_{i}$.
} 
and

$$
\begin{aligned}
& \frac{1}{\sqrt{n}} \sum_{i=1}^{n} t_{n}\left(x_{i} \zeta_{0}, \zeta_{0}\right) p_{i}^{\prime} \mathrm{E}\left(u_{1 i} \mid x_{i} \zeta_{0}\right) \frac{1}{B_{i}}\left(B_{n i}-B_{i}\right) \\
& =\frac{1}{\sqrt{n}} \sum_{i=1}^{n}\left\{I_{T}\left(x_{i} \zeta_{0}\right) \mathrm{E}\left(p_{i}^{\prime} \mid x_{i} \zeta_{0}\right) \mathrm{E}\left(u_{1 i} \mid x_{i} \zeta_{0}\right)\right. \\
& \left.\quad-\mathrm{E}\left[I_{T}\left(x_{i} \zeta_{0}\right) \mathrm{E}\left(p_{i}^{\prime} \mid x_{i} \zeta_{0}\right) \mathrm{E}\left(u_{1 i} \mid x_{i} \zeta_{0}\right)\right]\right\}+o_{p}(1) .
\end{aligned}
$$

Therefore,

$$
\begin{aligned}
& \frac{1}{\sqrt{n}} \sum_{i=1}^{n} t_{n}\left(x_{i} \zeta_{0}, \zeta_{0}\right) p_{i}^{\prime}\left(u_{1 i}-\mathrm{E}_{n}\left(u_{1} \mid x_{i} \zeta_{0}\right)\right) \\
& =\frac{1}{\sqrt{n}} \sum_{i=1}^{n} I_{T}\left(x_{i} \zeta_{0}\right)\left(p_{i}-\mathrm{E}\left(p_{i} \mid x_{i} \zeta_{0}\right)\right)^{\prime}\left(u_{1 i}-\mathrm{E}\left(u_{1 i} \mid x_{i} \zeta_{0}\right)\right)+o_{p}(1) .
\end{aligned}
$$

\section{References}

Amemiya, T., 1978, The estimation of a simultaneous equation generalized probit model, Econometrica 46, 1193-1206.

Amemiya, T., 1983, A comparison of the Amemiya GLS and the Lee-Maddala-Trost G2SLS in a simultaneous-equations tobit model, Journal of Econometrics 23, 295-300.

Bierens, H.J., 1985, Kernel estimators of regression functions, in: T.W. Bewley, ed., Advances in econometrics, Fifth world congress, Vol. 1 (Cambridge University Press, New York, NY) 99-144.

Blundell, R.W. and R.J. Smith, 1989, Estimation in a class of simultaneous equation limited dependent variable models, Review of Economic Studies 56, 37-58.

Chamberlain, G., 1986, Asymptotic efficiency in semi-parametric models with censoring, Journa] of Econometrics 32, 189-218.

Cosslett, S., 1987, Efficiency bounds for distribution-free estimators of the censored regression models, Econometrica 55, 559-585.

Heckman, J.J., 1979, Sample bias as specification error, Econometrica 47, 153-162.

Ichimura, H., 1987, Estimation of single index models, Ph.D. thesis (Department of Economics, MIT, Cambridge, MA).

Ichimura, H. and L.F. Lee, 1991, Semiparametric estimation of multiple index models: Single equation estimation, Ch. 1 in: W.A. Barnett, J. Powell, and G. Tauchen, eds., Nonparametric and semiparametric methods in econometrics and statistics (Cambridge University Press, New York, NY).

Klein, R.W. and R.H. Spady, 1987, An efficient semiparametric estimator for discrete choice models, Economics discussion paper no. 67 (Bellcore, Morristown, NJ).

LeCam, L., 1960, Locally asymptotically normal families of distributions, University of California Publications in Statistics 3, 37-98.

Lee, L.F., 1981, Simultaneous equations models with discrete endogenous variables, Ch. 9 in: C.F. Manski and D. McFadden, eds., Structural analysis of discrete data and econometric applications (MIT Press, Cambridge, MA).

Lee, L.F., 1990, Efficient semiparametric scoring estimation of sample selection models, Discussion paper no. 255 (Center for Economic Research, University of Minnesota, Minneapolis, MN). 
Lee, L.F., 1992, Semiparametric nonlinear least-squares estimation of truncated regression models, Econometric Theory 8, 52-94

Lee, L.F., G.S. Maddala, and R.P. Trost, 1980, Asymptotic covariance matrices of two-stage probit and two-stage tobit methods for simultaneous equations models with selectivity, Econometrica $48,491-503$.

Manski, C.F., 1984, Adaptive estimation of non-linear regression models, Econometric Reviews 3, 145-194.

Nadaraja, E., 1964, On regression estimator, Theory of Probability and Its Applications 9, 157-159.

Newey, W.K., 1987, Efficient estimation of limited dependent variable models with endogenous explanatory variables, Journal of Econometrics 36, 231-250.

Olsen, R.J., 1980, A least squares correction for selectivity bias, Econometrica 48, 1815-1820.

Powell, J.L., 1987, Semiparametric estimation of bivariate latent variable models, Discussion paper no. 8704 (Social Systems Research Institute, University of Wisconsin, Madison, WI).

Powell, J.L., J.H. Stock, and T.M. Stoker, 1989, Semiparametric estimation of index coefficients, Econometrica 57, 1403-1430.

Robinson, P.M., 1988, Root-n-consistent semiparametric regression, Econometrica 56, 931-954.

Serfling, R.J., 1980, Approximation theorems of mathematical statistics (Wiley, New York, NY).

Silverman, B.W., 1986, Density estimation for statistics and data analysis (Chapman and Hall, New York, NY).

Stoker, T.M., 1986, Consistent estimation of scaled coefficients, Econometrica 54, 1461-1481.

Watson, G.S., 1964, Smooth regression analysis, Sankha Series A 26, 101-116. 